\title{
Unearthing the Visions of a Master: Harmonic Maass Forms and Number Theory
}

\author{
Ken Ono
}

For my teacher Basil Gordon on his 75th birthday

\begin{abstract}
Together with his collaborators, most notably Kathrin Bringmann and Jan Bruinier, the author has been researching harmonic Maass forms. These non-holomorphic modular forms play central roles in many subjects: arithmetic geometry, combinatorics, modular forms, and mathematical physics. Here we outline the general facets of the theory, and we give several applications to number theory: partitions and $q$-series, modular forms, singular moduli, Borcherds products, extensions of theorems of Kohnen-Zagier and Waldspurger on modular $L$-functions, and the work of Bruinier and Yang on Gross-Zagier formulae. What is surprising is that this story has an unlikely beginning: the pursuit of the solution to a great mathematical mystery.
\end{abstract}

Modular forms are central in contemporary mathematics. Indeed, modular forms play crucial roles in algebraic number theory, algebraic topology, arithmetic geometry, combinatorics, number theory, representation theory, and mathematical physics. The recent history of the subject includes (to name a few) great successes on the Birch and Swinnerton-Dyer Conjecture, Mirror Symmetry, Monstrous Moonshine, and the proof of Fermat's Last Theorem. These celebrated works are dramatic examples of the evolution of mathematics; indeed, it would have been impossible to prophesy them fifty years ago.

Instead of travelling back in time to the 1950s, our story (also see [165]) begins in 1887, in a village in India. Our mathematics, ${ }^{1}$ which is about harmonic Maass forms, begins with the legend (see $[6,39,40,110,111,133$, 165]) of the great mathematician Srinivasa Ramanujan, and the mathematics he conjured from his death bed.

The author thanks the support of the NSF, the Manasse family, and the Hilldale Foundation.

${ }^{1}$ This is an exposition of the author's lectures at the '08 Harvard-MIT CDM Conference.

(C)2009 International Press 


\section{The gift from Kumbakonam}

Ramanujan was born on December 22, 1887 in Erode, a town about 250 miles southwest of Chennai (formerly known as Madras). He was a Brahmin, a member of India's priestly caste. He grew up in Kumbakonam, a small town roughly 150 miles south of Chennai, where his father Srinivasa was a cloth merchant's clerk. Kumbakonam, which is situated on the banks of the sacred Cauvery River, was (and remains today) a cosmopolitan center of the rural Indian district of Tanjore in the state of Tamil Nadu.

As a boy, Ramanujan was a stellar student. Indeed, he won many awards $^{2}$ at Town High School in Kumbakonam. Thanks to his exemplary performance, Ramanujan won a scholarship to Government College. His life took a dramatic turn when a friend loaned him a copy of G. S. Carr's Synopsis of elementary results in pure mathematics, which G. H. Hardy would later describe (see page 3 of $[\mathbf{1 1 1}]$ ) as

“...the 'synopsis' it professes to be. It contains enunciations' of 6165 theorems, systematically and quite scientifically arranged, with proofs which are often little more than cross-references..."

The amateur Ramanujan became infatuated with mathematics research, recording his "discoveries" in notebooks, imitating Carr's format. He typically offered no proofs of any kind. His addiction for mathematics made it impossible for him to focus on schoolwork at Government College, and he unceremoniously flunked out. He would later get a second chance, a scholarship to attend Pachaiyappa's College in Madras. His obsession again kept him from his school work, and he flunked out a second time (see [41]).

By 1907, the gifted Ramanujan was an academic failure. There was no room for him in India's system of higher education. His friends and parents must have recognized his genius, and, to their credit, they continued to support him by allowing him to work on mathematics full bore. There are vivid accounts (for example, see page 67 of [133]) of Ramanujan hunched over his slate on the porch of his house, and in the halls of Sarangapani Temple working feverishly on mathematics.

"....Ramanujan would sit working on the pial (porch) of his house..., legs pulled into his body, a large slate spread across his lap, madly scribbling,... When he figured something out, he sometimes seemed to talk to himself, smile, and shake his head with pleasure."

For the next few years, Ramanujan continued his research in near isolation. ${ }^{4}$ Thankfully, Ramanujan secured a job as a clerk at the Madras Port Trust which provided a salary, and left time for mathematics. Although

\footnotetext{
${ }^{2}$ Present day visitors to Town High School can view copies of these treasured awards.

${ }^{3}$ B. C. Berndt has informed me that there are actually 4417 theorems in Carr's book.

${ }^{4} \mathrm{He}$ did share his work with S. Narayana Aiyar, a friend who earned a Master's in mathematics.
} 
some Indian patrons acknowledged his genius, he was unable to find suitable mentors since Indian mathematicians did not understand his work. He was unable to share his passion and raison d'être with others.

After years in purgatory, Ramanujan boldly wrote distinguished English mathematicians. He wrote M. J. M. Hill, and then H. F. Baker and E. W. Hobson, without success. Then on January 16, 1913, he wrote G. H. Hardy, a young analyst and number theorist at Cambridge University. With his letter he included nine pages of mathematical scrawl. C. P. Snow later elegantly recounted (see pages 30-33 of [112]) Hardy's reaction to the letter:

"One morning in 1913, he (Hardy) found, among the letters on his breakfast table, a large untidy envelope decorated with Indian stamps. When he opened it...he found line after line of symbols. He glanced at them without enthusiasm. He was by this time... a world famous mathematician, and ...he was accustomed to receiving manuscripts from strangers. .... The script appeared to consist of theorems, most of them wild or fantastic... There were no proofs of any kind... A fraud or genius? ... is a fraud of genius more probable than an unknown mathematician of genius? ...He decided that Ramanujan was, in terms of ...genius, in the class of Gauss and Euler..."

In response to the letter, Hardy invited Ramanujan to Cambridge. Although Hindu beliefs forbade such travel at the time, Ramanujan's mother had a vision from Goddess Namagiri granting him permission to accept the invitation. Ramanujan left his life in south India for Cambridge University, home of some of the world's most distinguished mathematicians. He arrived on April 14, 1914.

Over the next five years, Ramanujan published extensively on a wide variety of topics: hypergeometric series, elliptic functions, modular forms, probabilistic number theory, the theory of partitions and $q$-series, among others. He would write over thirty papers, including seven with Hardy. He was named a Fellow of Trinity College, and he was elected a Fellow of the Royal Society (F.R.S.). News of his election spread quickly, and in India he was hailed as a national hero.

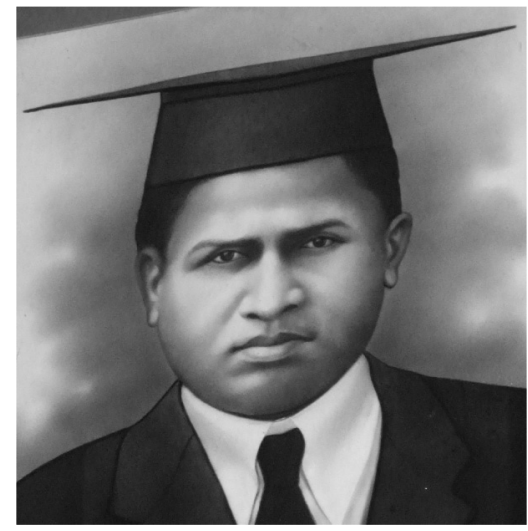

Ramanujan at his Master's graduation ceremony 
Sadly, the story of Ramanujan's life ends tragically. He fell ill towards the end of his stay in England. He returned to south India in the spring of 1919 seeking a return to health and a forgiving climate. Unfortunately, his health steadily declined over the course of the ensuing year, and he died on April 26, 1920 in Madras. He was thirty two years old. Amazingly, despite his illness, he spent the last year of his life, again in mathematical isolation, conjuring a most beautiful theory, one which was nearly lost to the world.

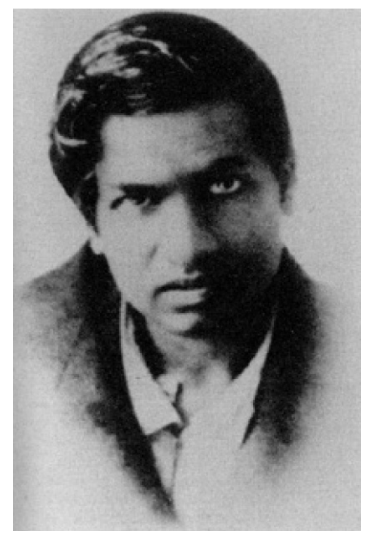

Ramanujan's 1919 passport photograph ${ }^{5}$

The legend of Ramanujan has continued to grow with the ever-increasing importance of his mathematics. To explain, it is appropriate to recall Hardy's own words ${ }^{6}$ written shortly after Ramanujan's death:

"Opinions may differ about the importance of Ramanujan's work, the kind of standard by which it should be judged, and the influence which it is likely to have on mathematics of the future. ... He would probably have been a greater mathematician if he could have been caught and tamed a little in his youth. On the other hand he would have been less of a Ramanujan, and more of a European professor, and the loss might have been greater than the gain..."

In view of the last eighty five years of progress in number theory, it is clear that the loss would have been much greater than the gain. On one hand, as Hardy did, we may largely base our conclusion on the contents of Ramanujan's notebooks, which, apart from the "lost notebook" that contained the work of his last year, were known to mathematicians at the time of his death. They are a repository of thousands of cryptic entries on evaluations and identities of strangely named functions. Through the tireless efforts of B. C. Berndt, adding to the accumulated effort of earlier mathematicians such as Hardy, G. N. Watson, B. M. Wilson, and R. A. Rankin, a clear picture has emerged which reveals Ramanujan's incredible gift for

\footnotetext{
${ }^{5}$ These images illustrate Ramanujan's poor health in 1919 . He seems to have lost considerable weight.

${ }^{6}$ See page xxxvi of $[\mathbf{1 1 0}]$.
} 
formulas and combinatorial relations (see $[32,33,34,35,36,111,175]$ ). Perhaps he was the greatest such mathematician of all time.

On the other hand, this flattering assessment is grossly inadequate, for it does not take into account Ramanujan's impact on Hardy's "mathematics of the future". Indeed, number theory has undergone a tremendous evolution since Ramanujan's death, and today it bears no resemblance to the number theory of his day. The subject is now dominated by the arithmetic and analytic theory of automorphic and modular forms, the study of Diophantine questions under the rubric of arithmetical algebraic geometry, and the emergence of computational number theory and its applications. These subjects boast many of the most celebrated achievements of 20th century mathematics such as: Deligne's proof of the Weil Conjectures, the solution to Gauss' Class Number Problem by Goldfeld, Gross, and Zagier, Wiles' proof of Fermat's Last Theorem, and Borcherds's work on the infinite product expansions of automorphic forms. A proper assessment of Ramanujan's greatness must then take into account the remarkable fact that his work, the portion which was known to Hardy, makes intimate contact with all of these notable achievements. Clearly, Ramanujan was a great anticipator. ${ }^{7}$ His work provided examples of deeper structures, and suggested important questions which are now inescapable in the panorama of modern number theory (see $[\mathbf{1 6 5}]$ ).

This brings us to the mysterious mathematics Ramanujan developed during his last year, which was nearly lost. For nearly 60 years, the only information about this work was contained in the surviving portion of Ramanujan's last letter to Hardy, dated January 20, 1920. The task of teasing his theory from this letter has been one of the greatest challenges for Ramanujan historians. Then in 1976, G. E. Andrews unearthed Ramanujan's "lost notebook", the sheafs of mathematical scrawl containing the results Ramanujan described in this last letter. What are the secrets of the mathematical scrawl Ramanujan penned during his last days? What is its impact on Hardy's "mathematics of the future"?

\section{Ramanujan's playground}

The new mathematics described in this paper is born from the enigmatic theory Ramanujan conjured during the last year of his life. To set the stage, it makes sense to first ponder some of Ramanujan's early work which we think led him to conceive this theory.

At first glance, the stuff of partitions seems like mere child's play:

$$
4=3+1=2+2=2+1+1=1+1+1+1 .
$$

Therefore, there are 5 partitions of the number 4 . As happens in number theory, seemingly simple problems, such as the business of adding and counting, can quickly lead to difficult and beautiful problems (for example,

\footnotetext{
${ }^{7}$ I first heard Ramanujan described this way by Manjul Bhargava.
} 
see $[4, \mathbf{1 6}, \mathbf{2 4}])$. A partition of the natural number $n$ is any non-increasing sequence of natural numbers whose sum is $n$. The number of partitions of $n$ is denoted by $p(n)$ (by convention, we let $p(0):=1$ ).

2.1. Ramanujan's work on partitions. Ramanujan was perhaps the first mathematician to seriously investigate the properties of this function $p(n)$. He sought a formula for $p(n)$, one which describes the phenomenal rate of growth suggested by the table below.

\begin{tabular}{cc}
\hline \hline$n$ & $p(n)$ \\
\hline & \\
0 & 1 \\
1 & 1 \\
2 & 2 \\
3 & 3 \\
4 & 5 \\
5 & 7 \\
$\vdots$ & $\vdots$ \\
50 & 204226 \\
$\vdots$ & $\vdots$ \\
200 & $\vdots$ \\
$\vdots$ & 3972999029388 \\
1000 & 24061467864032622473692149727991 \\
\hline
\end{tabular}

Together with Hardy, Ramanujan gave a remarkable asymptotic formula $[\mathbf{1 1 3}, \mathbf{1 1 4}]$, which was perfected by $\mathrm{H}$. Rademacher $[\mathbf{1 6 8}, \mathbf{1 6 9}]$ two decades later to obtain a formula which is so accurate that it can be used to compute individual values of $p(n)$. More precisely, Rademacher defined explicit functions $T_{k}(n)$ such that for all positive $n$ we have

$$
p(n)=\sum_{k=1}^{\infty} T_{k}(n) .
$$

The function $T_{1}(n)$ alone gives the Hardy-Ramanujan asymptotic formula

$$
p(n) \sim \frac{1}{4 n \sqrt{3}} \cdot e^{\pi \sqrt{2 n / 3}} .
$$

The rate at which Rademacher's series converges is astonishing; for example, the first eight terms give the approximation

$$
p(200) \approx 3,972,999,029,388.004,
$$

which nicely compares with the exact value in the table above. These works stand out further in importance since they mark the birth of the circle method, now one of the fundamental tools in analytic number theory.

Ramanujan also investigated the divisibility properties of $p(n)$. At first glance, the combinatorial definition of the partition function gives no reason 
to believe that it possesses any interesting arithmetic properties. There is nothing, for example, which would lead us to think that $p(n)$ should exhibit a preference to be even rather than odd. A natural suspicion might be that the values of $p(n)$ are distributed evenly modulo 2. Numerics seem to confirm this suspicion, as well as its obvious generalization when 2 is replaced by 3 . However, when we replace 3 by 5 , something quite different happens; we discover that 3,611 (many more than the expected one-fifth) of the first 10,000 values of $p(n)$ are divisible by 5 .

The explanation must have been clear to Ramanujan when he first saw the famous table of values of $p(n)$ computed by P. A. MacMahon, a wellknown combinatorialist and major in the British Royal Artillery. These values, starting with $n=0$, were listed in five columns, and so Ramanujan would have seen something like the following.

$\begin{array}{ccccc}1 & 1 & 2 & 3 & 5 \\ 7 & 11 & 15 & 22 & 30 \\ 42 & 56 & 77 & 101 & 135 \\ 176 & 231 & 297 & 385 & 490 \\ 627 & 792 & 1002 & 1255 & 1575 \\ 1958 & 2436 & 3010 & 3718 & 4565 .\end{array}$

What is striking, of course, is that every entry in the last column is a multiple of 5. This phenomenon, which persists, explains the apparent aberration above, and was the first of Ramanujan's ground-breaking discoveries on the arithmetic of $p(n)$. He proved $[\mathbf{1 7 3}, \mathbf{1 7 4}]$, for every non-negative integer $n$, that

$$
\begin{aligned}
& p(5 n+4) \equiv 0 \quad(\bmod 5), \\
& p(7 n+5) \equiv 0 \quad(\bmod 7), \\
& p(11 n+6) \equiv 0 \quad(\bmod 11) .
\end{aligned}
$$

Ramanujan conjectured (and in some cases proved) $[\mathbf{3 8}, \mathbf{1 7 3}, \mathbf{1 7 4}]$ that there are further congruence properties in which the moduli are powers of 5,7 , or 11 .

Subsequent works $[\mathbf{2 8}, \mathbf{2 0 8}]$ by A. O. L. Atkin and Watson resolved the conjectured congruences of Ramanujan, and in an important paper [29] Atkin discovered completely new congruences modulo some further small primes. The author proved [162] that Atkin's phenomenon generalizes to all prime moduli exceeding 3, and in later work Ahlgren and the author further extended $[\mathbf{2}, \mathbf{3}]$ these results to include all moduli coprime to 6 . In 
particular, it turns out that there are such Ramanujan-type congruences for every modulus $M$ coprime to 6 . For example, we have that

$$
p(4063467631 n+30064597) \equiv 0 \quad(\bmod 31) .
$$

This comprehensive theory requires deep works of Deligne, Serre, and Shimura $[83,187,197]$.

2.2. Ramanujan's playground is a testing ground. Ramanujan's work on $p(n)$, and the research it inspired, underscores the fact that the theory of partitions has historically served as a delightful "testing ground" for some of the deepest developments in the theory of modular forms. Indeed, (2.1) and (2.2) are the first triumphs of the circle method, while congruences such as (2.3) arise from the interplay between the Deligne-Serre theory of $\ell$-adic Galois representations, the "language" of the proof of Fermat's Last Theorem, and Shimura's theory of half-integral weight modular forms.

To make this connection between partitions and modular forms, we simply require Euler's combinatorial generating function

$$
\sum_{n=0}^{\infty} p(n) q^{n}=\prod_{n=1}^{\infty} \frac{1}{1-q^{n}}=1+q+2 q^{2}+3 q^{3}+5 q^{4}+7 q^{5}+\cdots .
$$

This formal power series is essentially a "weakly holomorphic modular form".

To make this a bit more precise, let $\Gamma \subset \mathrm{SL}_{2}(\mathbb{Z})$ be a subgroup. An element $\gamma=\left(\begin{array}{ll}a & b \\ c & d\end{array}\right) \in \mathrm{SL}_{2}(\mathbb{Z})$ acts on $\mathbb{H}$, the upper-half of the complex plane, by the linear fractional transformation $\gamma z:=\frac{a z+b}{c z+d}$. Loosely speaking, a weight $k$ modular form on $\Gamma$ is a holomorphic function $f$ on $\mathbb{H}$ which satisfies

$$
f(\gamma z)=(c z+d)^{k} f(z)
$$

for all $\gamma \in \Gamma$, which in addition is holomorphic "at the cusps". A weakly holomorphic modular form satisfies this definition but is permitted to have poles at cusps.

At the heart of the matter is Dedekind's weight $1 / 2$ modular form, the eta-function:

$$
\eta(z):=q^{1 / 24} \prod_{n=1}^{\infty}\left(1-q^{n}\right),
$$

where $q:=e^{2 \pi i z}$. For $z \in \mathbb{H}$, it turns out that

$$
\eta(z+1)=e^{\frac{\pi i}{12}} \eta(z) \text { and } \eta(-1 / z)=(-i z)^{\frac{1}{2}} \eta(z) .
$$

Since $\mathrm{SL}_{2}(\mathbb{Z})$ is generated by

$$
T:=\left(\begin{array}{ll}
1 & 1 \\
0 & 1
\end{array}\right) \quad \text { and } \quad S:=\left(\begin{array}{cc}
0 & -1 \\
1 & 0
\end{array}\right)
$$


it follows that $\eta(z)$ is a weight $1 / 2$ modular form on $\mathrm{SL}_{2}(\mathbb{Z})$ with a "multiplier system". Since $\eta(z)$ is nonvanishing on $\mathbb{H},(2.4)$ then implies that

$$
\frac{1}{\eta(z)}=\sum_{n=0}^{\infty} p(n) q^{n-\frac{1}{24}}
$$

is a weight $-1 / 2$ weakly holomorphic modular form.

2.3. How did Ramanujan think of modular forms? Since we view Ramanujan's results on the partition function, and the research it inspired, as a testing ground for the theory of modular forms, we are compelled to ask how Ramanujan thought of modular forms. A brief inspection of most of his works (for example, see $[\mathbf{3 8}, \mathbf{1 7 5}]$ ) suggests that his view was consistent with the classical treatment found in most textbooks (for example, see [71, $84,125,134,143,155,164,177,185,193,196])$ on modular forms. Indeed, the bulk of his work on modular forms depends on the properties of the $q$-series

$$
\begin{aligned}
& P(q):=1-24 \sum_{n=1}^{\infty} \sigma_{1}(n) q^{n}, \\
& Q(q):=1+240 \sum_{n=1}^{\infty} \sigma_{3}(n) q^{n}, \\
& R(q):=1-504 \sum_{n=1}^{\infty} \sigma_{5}(n) q^{n},
\end{aligned}
$$

(where $\sigma_{\nu}(n):=\sum_{d \mid n} d^{\nu}$ ), which are the classical Eisenstein series $E_{2}(z)$, $E_{4}(z)$ and $E_{6}(z)$ from the theory of modular forms. Therefore, much of his work followed classical lines.

This assessment, however, ignores Ramanujan's extensive work on combinatorial $q$-series, which at first glance have nothing to do with the theory of modular forms. Ramanujan, a master manipulator of power series, thought deeply about combinatorial power series such as infinite products, which are often modular like Dedekind's eta-function $\eta(z)$, and Jacobi's identity

$$
\prod_{n=1}^{\infty}\left(1-q^{2 n}\right)\left(1+t^{2} q^{2 n-1}\right)\left(1+t^{-2} q^{2 n-1}\right)=\sum_{n \in \mathbb{Z}} t^{2 n} q^{n^{2}} .
$$

He also thought deeply about power series he referred to as Eulerian series, such as

$$
\Omega(t ; q):=1+\sum_{n=1}^{\infty} \frac{q^{n^{2}}}{(1-t q)^{2}\left(1-t q^{2}\right)^{2} \cdots\left(1-t q^{n}\right)^{2}},
$$

which seems to have nothing to do with modular forms. However, in some rare cases such series turn out to coincide with modular forms.

We now give one such coincidence, an identity which is particularly relevant for Ramanujan's work on partitions. We first recall some elementary 
combinatorial notions. One may visualize a partition $\lambda_{1}+\lambda_{2}+\cdots+\lambda_{k}$ as a Ferrers diagram, a left justified array of dots consisting of $k$ rows in which there are $\lambda_{i}$ dots in the $i$ th row:

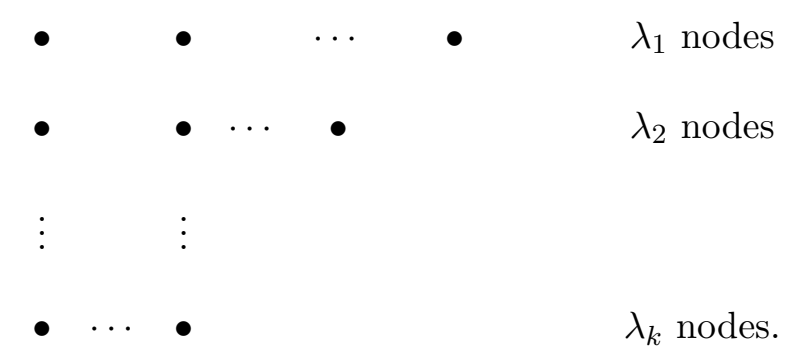

The Durfee square of such a Ferrers diagram is the largest square of nodes in the upper left hand corner of the diagram. The boundary of a Durfee square naturally then divides a partition into a perfect square and two partitions whose parts do not exceed the side length of the Durfee square.

ExAMPLE 2.1. The Ferrers diagram of the partition $5+5+3+3+2+1$ is:

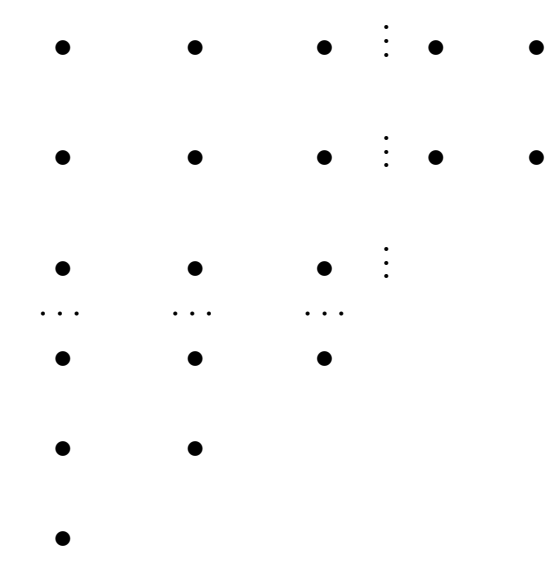

Therefore, this partition decomposes as the Durfee square of size 9 , and the two partitions $2+2$, and $3+2+1$.

Armed with these notions, we prove the following $q$-series identity for the generating function for $p(n)$.

THEOREM 2.2. The following combinatorial identity is true:

$\sum_{n=0}^{\infty} p(n) q^{n}=\prod_{n=1}^{\infty} \frac{1}{1-q^{n}}=1+\sum_{m=1}^{\infty} \frac{q^{m^{2}}}{(1-q)^{2}\left(1-q^{2}\right)^{2} \cdots\left(1-q^{m}\right)^{2}}=\Omega(1 ; q)$.

In particular, we have that

$$
\frac{1}{\eta(z)}=q^{-\frac{1}{24}} \prod_{n=1}^{\infty} \frac{1}{1-q^{n}}=q^{-\frac{1}{24}}+\sum_{m=1}^{\infty} \frac{q^{m^{2}-\frac{1}{24}}}{(1-q)^{2}\left(1-q^{2}\right)^{2} \cdots\left(1-q^{m}\right)^{2}} .
$$


Proof. For every positive integer $m$, the $q$-series

$$
\frac{1}{(1-q)\left(1-q^{2}\right) \cdots\left(1-q^{m}\right)}=\sum_{n=0}^{\infty} a_{m}(n) q^{n}
$$

is the generating function for $a_{m}(n)$, the number of partitions of $n$ whose summands do not exceed $m$. Therefore by the discussion above, the $q$-series

$$
\frac{q^{m^{2}}}{(1-q)^{2}\left(1-q^{2}\right)^{2} \cdots\left(1-q^{m}\right)^{2}}=\sum_{n=0}^{\infty} b_{m}(n) q^{n}
$$

is the generating function for $b_{m}(n)$, the number of partitions of $n$ whose Ferrers diagram has a Durfee square of size $m^{2}$. The theorem follows by summing in $m$.

Although they are quite rare, there are further examples of such $q$-series identities in which an Eulerian series is essentially a modular form. Among them, perhaps the most famous are the Rogers-Ramanujan identities [171, $182,183,184]$ :

$$
\begin{aligned}
& \prod_{n=0}^{\infty} \frac{1}{\left(1-q^{5 n+1}\right)\left(1-q^{5 n+4}\right)}=1+\sum_{n=1}^{\infty} \frac{q^{n^{2}}}{(1-q)\left(1-q^{2}\right) \cdots\left(1-q^{n}\right)}, \\
& \prod_{n=0}^{\infty} \frac{1}{\left(1-q^{5 n+2}\right)\left(1-q^{5 n+3}\right)}=1+\sum_{n=1}^{\infty} \frac{q^{n^{2}+n}}{(1-q)\left(1-q^{2}\right) \cdots\left(1-q^{n}\right)} .
\end{aligned}
$$

These infinite products are essentially the Fourier expansions of weight 0 weakly holomorphic modular forms, the type studied classically by Jacobi, Klein, and Siegel, and again more recently by Kubert and Lang in their work on modular units (for example, see $[\mathbf{1 3 9}, \mathbf{1 8 6}]$ ).

REMARK 1. The literature on such identities is extensive (for example, see works by Andrews, Gordon, Göllnitz, and Slater $[\mathbf{8}, \mathbf{1 0 2}, \mathbf{1 0 1}, \mathbf{1 9 5}]$ to name a few), and the pursuit of further identities and their interpretations remains an active area of research largely due to applications in combinatorics, Lie theory, number theory and physics (for example, see $[\mathbf{1 3}],[\mathbf{1 2 9}]$ and $[\mathbf{1 4 5}]$ to name a few). In this direction, W. Nahm $[\mathbf{1 5 9}]$ has very interesting work related to the question of when a basic hypergeometric-type series is automorphic.

These identities stand out since there is no reason to believe, for function theoretic reasons, that an Eulerian series should ever be modular. Indeed, there is no general theory of transformation laws for Eulerian series.

It is not difficult to imagine Ramanujan's mindset. Understanding this quandary, it seems that Ramanujan, largely motivated by his work on partitions and the Rogers-Ramanujan identities, spent the last year of his life thinking deeply about the "near" modularity of Eulerian series. He understood the importance of developing a "new theory", one which overlaps in spots with the classical theory of modular forms.

He discovered the mock theta functions. 


\section{Ramanujan's last letter to Hardy}

Hardy's romantic story of Ramanujan opens with the delivery of the landmark letter from Ramanujan in 1913. Our tale begins with Ramanujan's last letter to Hardy, dated January 12, 1920, just three months before his death. We quote (see pages 220-224 of [39]):

"I am extremely sorry for not writing you a single letter up to now... I discovered very interesting functions recently which I call "Mock" $\vartheta$-functions. Unlike the "False" $\vartheta$-functions (studied partially by Prof. Rogers in his interesting paper) they enter into mathematics as beautifully as the ordinary theta functions. I am sending you with this letter some examples."

This letter contained 17 examples including:

$$
\begin{aligned}
f(q) & :=1+\sum_{n=1}^{\infty} \frac{q^{n^{2}}}{(1+q)^{2}\left(1+q^{2}\right)^{2} \cdots\left(1+q^{n}\right)^{2}}, \\
\omega(q) & :=\sum_{n=0}^{\infty} \frac{q^{2 n^{2}+2 n}}{(1-q)^{2}\left(1-q^{3}\right)^{2} \cdots\left(1-q^{2 n+1}\right)^{2}}, \\
\lambda(q) & :=\sum_{n=0}^{\infty} \frac{(-1)^{n}(1-q)\left(1-q^{3}\right) \cdots\left(1-q^{2 n-1}\right) q^{n}}{(1+q)\left(1+q^{2}\right) \cdots\left(1+q^{n-1}\right)} .
\end{aligned}
$$

At first glance, these series indeed seem to resemble the Eulerian series in Theorem 2.2 and (2.8). For example, in terms of (2.7), we have that both

$$
f(q)=\Omega(-1 ; q) \quad \text { and } \quad \frac{1}{\eta(z)}=q^{-\frac{1}{24}} \Omega(1 ; q) .
$$

However, as Ramanujan asserts, series such as $f(q), \omega(q)$ and $\lambda(q)$ are not modular; they are what he calls mock theta functions.

Most of the surviving text of the letter, which amounts to roughly 4 typewritten pages, consists of explicit formulas for these 17 strange formal power series. The theoretical content is rather obtuse, and consists of an elementary discussion on the asymptotics of Eulerian series and their behavior near points on the unit disk. He gives no indication of how he derived his 17 examples. He even divides these examples into groups based on their "order", a term he never defines. With such flimsy clues, how could one rederive Ramanujan's theory? What did he mean by a mock theta function?

Despite these formidable challenges, a few mathematicians such as G. E. Andrews, L. Dragonette, ${ }^{8}$ A. Selberg, and Watson $[\mathbf{7}, \mathbf{8 5}, \mathbf{1 9 0 ,}$ 206, 207] investigated Ramanujan's mock theta functions for what they were, a list of enigmatic power series. For example, Andrews and Dragonette

\footnotetext{
${ }^{8}$ Leila Dragonette is better known under her married name, Leila Bram.
} 
$[7,85]$ investigated Ramanujan's claimed asymptotic formula for the mock theta function

$$
f(q)=\Omega(-1 ; q)=\sum_{n=0}^{\infty} \alpha(n) q^{n}:=1+\sum_{n=1}^{\infty} \frac{q^{n^{2}}}{(1+q)^{2}\left(1+q^{2}\right)^{2} \cdots\left(1+q^{n}\right)^{2}} .
$$

They proved, for positive $n$, that

$$
\alpha(n) \sim \frac{(-1)^{n-1}}{2 \sqrt{n-\frac{1}{24}}} \cdot e^{\pi \sqrt{\frac{n}{6}-\frac{1}{144}}} .
$$

REMARK 2. Strictly speaking, Dragonette first proved the asymptotic [85], while Andrews refined her work to obtain [7] the asymptotic with an improved error term.

Despite the absence of a theory, or much less, just a simple useful definition of a mock theta function, these few early works bolstered the belief that Ramanujan had discovered something important. Watson, in his own words, ${ }^{9}$ proclaimed [206]:

"Ramanujan's discovery of the mock theta functions makes it obvious that his skill and ingenuity did not desert him at the oncoming of his untimely end. As much as any of his earlier work, the mock theta functions are an achievement sufficient to cause his name to be held in lasting remembrance. To his students such discoveries will be a source of delight and wonder until the time shall come when we too shall make our journey to that Garden of Proserpine (a.k.a. Persephone)..."

\section{The "lost notebook" and Dyson's "challenge for the future"}

By the mid 1970s, little progress had been made on the mock theta functions. They remained a list of enigmatic power series, without any apparent connection to the theory of modular forms, or any other comprehensive theory for that matter.

Then in the spring of 1976, Andrews discovered Ramanujan's "lost notebook" in an old box of papers hidden away in the Trinity College Library at Cambridge University. This notebook, consisting of over 100 pages of mathematical scrawl [176], was archived among papers from Watson's estate. Miraculously, the "lost notebook" had somehow survived a circuitous journey from India in the early 1920s to lie forgotten in the Trinity College Library archives. The journey was indeed miraculous, for the contents of the box almost met a catastrophic end in 1968 when Rankin saved them just a few days before they were scheduled to be burned. Although the

\footnotetext{
${ }^{9}$ This text is from Watson's 1936 Presidential Address to the London Mathematical Society.
} 
manuscript was never truly lost, it was long forgotten, and buried among Watson's random papers. Andrews proclaimed [9]:

"... the fact that its existence was never mentioned by anyone for over 55 years leads me to call it "lost".

The discovery of the "lost notebook" was the catalyst which made it possible to begin chipping away at the puzzle of Ramanujan's mock theta functions. On top of giving 2 further mock theta functions, adding to the 17 from the last letter and 3 defined by Watson [206], the pages contained many clues: striking identities and relations. As usual, these were given without proof.

To illustrate the value of these clues, we consider some examples of Ramanujan's claimed identities, the famous "mock theta conjectures." To state them, we first fix notation. For non-negative integers $n$, let

$$
(x)_{n}:=(x ; q)_{n}:=\prod_{j=0}^{n-1}\left(1-x q^{j}\right),
$$

and let

$$
(x)_{\infty}:=(x ; q)_{\infty}:=\prod_{j=0}^{\infty}\left(1-x q^{j}\right),
$$

where an empty product equals 1 . Let $f_{0}(q), f_{1}(q), \Phi(q)$, and $\Psi(q)$ be the mock theta functions

$$
\begin{aligned}
f_{0}(q) & :=\sum_{n=0}^{\infty} \frac{q^{n^{2}}}{(-q)_{n}}, \\
\Phi(q) & :=-1+\sum_{n=0}^{\infty} \frac{q^{5 n^{2}}}{\left(q ; q^{5}\right)_{n+1}\left(q^{4} ; q^{5}\right)_{n}} \\
f_{1}(q) & :=\sum_{n=0}^{\infty} \frac{q^{n^{2}+n}}{(-q)_{n}}, \\
\Psi(q) & :=-1+\sum_{n=0}^{\infty} \frac{q^{5 n^{2}}}{\left(q^{2} ; q^{5}\right)_{n+1}\left(q^{3} ; q^{5}\right)_{n}} .
\end{aligned}
$$

These $q$-series resemble (2.7) and the Eulerian series in the RogersRamanujan identities (2.8). The mock theta conjectures are a list of ten identities involving these 4 functions. Thanks to work of Andrews and F. Garvan [26], these ten identities follow from the truth of the following pair of identities.

Conjecture (Mock Theta Conjectures). The following identities are true:

$$
\begin{aligned}
& \frac{\left(q^{5} ; q^{5}\right)_{\infty}\left(q^{5} ; q^{10}\right)_{\infty}}{\left(q ; q^{5}\right)_{\infty}\left(q^{4} ; q^{5}\right)_{\infty}}=f_{0}(q)+2 \Phi\left(q^{2}\right) \\
& \frac{\left(q^{5} ; q^{5}\right)_{\infty}\left(q^{5} ; q^{10}\right)_{\infty}}{\left(q^{2} ; q^{5}\right)_{\infty}\left(q^{3} ; q^{5}\right)_{\infty}}=f_{1}(q)+2 q^{-1} \Psi\left(q^{2}\right) .
\end{aligned}
$$


These surprising identities equate simple infinite products to linear combinations of mock theta functions. These infinite products, like those in Theorem 2.2 and (2.8), are well known to essentially coincide with the Fourier expansions of certain weakly holomorphic modular forms. Therefore, the truth of these identities directly related mock theta functions to modular forms. These clues from the "lost notebook" finally placed Ramanujan's mock theta functions in the vicinity of the theory of modular forms.

Unfortunately, these clues would not prove to be enough to rederive Ramanujan's theory. Indeed, mathematicians even had to wait 10 years, until the work of D. Hickerson [118], just for a proof of these particularly vicious $q$-series identities.

Nonetheless, Andrews's discovery of the "lost notebook" in 1976 almost immediately sparked an explosion of research on the mock theta functions, largely spearheaded by Andrews. By the late 1990s, works by Andrews, Y.-S. Choi, H. Cohen, F. Dyson, Garvan, B. Gordon, Hickerson, R. McIntosh, M. Wakimoto $[9,11,12,14,15,21,26,27,72,73,74,75,76,80$, $103,104,118,119,204]$, among numerous others, revealed many of the deeper properties of the mock theta functions. Thanks to these works, and too many others to list, Ramanujan's 22 mock theta functions had been related to a surprising collection of subjects:

- Artin $L$-functions in number theory

- Hypergeometric functions

- Partitions

- Lie theory

- Mordell integrals

- Modular forms

- Polymer chemistry

-...

Despite this flurry of activity, the essence of Ramanujan's theory remained a mystery. The puzzle of his last letter to Hardy, thanks to the "lost notebook," had morphed into the enigmatic web of Ramanujan's 22 mock theta functions. The presence of this web strongly suggested the existence of a theory, and it also demanded a solution. In his plenary address at the Ramanujan Centenary Conference at the University of Illinois in 1987, Freeman Dyson beautifully summed up the situation [91]:

"The mock theta-functions give us tantalizing hints of a grand synthesis still to be discovered. Somehow it should be possible to build them into a coherent group-theoretical structure, analogous to the structure of modular forms which Hecke built around the old theta-functions of Jacobi. This remains a challenge for the future. My dream is that I will live to see the day when our young physicists, struggling to bring the predictions of superstring theory into correspondence with the facts of nature, will be led to enlarge their analytic machinery to include mock theta-functions...But before this can happen, the 
purely mathematical exploration of the mock-modular forms and their mocksymmetries must be carried a great deal further."

\section{Back to the future}

By the late 1990s, the vast literature on Ramanujan's mock theta functions contained many important clues for Dyson's "challenge for the future". In addition to the identities comprising the mock theta conjectures, there were further clues such as $q$-series identities relating mock theta functions to Lambert-type series and indefinite theta series. We recall two such identities involving the mock theta functions $f(q)$ and $f_{0}(q)$ (see (3.1) and (4.3)). In 1936, Watson [206] proved that

$f(q)=1+\sum_{n=1}^{\infty} \frac{q^{n^{2}}}{(1+q)^{2}\left(1+q^{2}\right)^{2} \cdots\left(1+q^{n}\right)^{2}}=\frac{2}{(q)_{\infty}} \cdot \sum_{n \in \mathbb{Z}} \frac{(-1)^{n} q^{\left(3 n^{2}+n\right) / 2}}{1+q^{n}}$,

and in 1986 Andrews [12] proved that

$$
f_{0}(q)=\sum_{n=0}^{\infty} \frac{q^{n^{2}}}{(-q)^{n}}=\frac{1}{(q)_{\infty}} \cdot\left(\sum_{\substack{n+j \geq 0 \\ n-j \geq 0}}-\sum_{\substack{n+j<0 \\ n-j<0}}\right)(-1)^{j} q^{\frac{5}{2} n^{2}+\frac{1}{2} n-j^{2}} .
$$

Such identities served as motivation for the 2002 Ph.D. thesis, written under the direction of D. Zagier, of S. Zwegers [218, 219]. Indeed, Zwegers researched the following two questions of Zagier (see page 2 of [219]):

(1) How do the mock $\vartheta$-functions fit in the theory of modular forms?

(2) Is there a theory of indefinite theta functions?

His thesis brilliantly addressed these questions by combining and extending ideas from a number of sources such as works of Lerch $[\mathbf{1 4 6 , 1 4 7}]$ on the functions

$$
\sum_{n \in \mathbb{Z}} \frac{(-1)^{n} e^{\pi i\left(n^{2}+n\right) \tau+2 \pi i n \nu}}{1-e^{2 \pi i n \tau+2 \pi i u}}
$$

where $\tau \in \mathbb{H}, \nu \in \mathbb{C}, u \in \mathbb{C} /(\mathbb{Z} \tau+\mathbb{Z})$, work of L. Göttsche and Zagier on indefinite $\vartheta$-functions [105], the theory of Jacobi forms [93], Mordell integrals $[157,158]$, and works of Andrews $[10,12,14,15]$.

Zwegers related Ramanujan's mock theta functions to real analytic vector-valued modular forms. Loosely speaking, he "completed" Ramanujan's mock theta functions by adding a non-holomorphic function, a so-called period integral, to obtain real analytic functions which obey desired modular transformation laws. We shall recount some of his work ${ }^{10}$ in Section 6 . The real analytic modular forms of Zwegers turned out to be examples of harmonic Maass forms (see Section 7.1 for the definition) which were defined

\footnotetext{
${ }^{10}$ Zagier delivered a Séminaire Bourbaki lecture on these recent works on Ramanujan's mock theta functions in 2007 [213].
} 
about the same time by Bruinier and Funke [63], a coincidence which catalyzed much of the research described here.

These developments sparked an immediate explosion in a wide number of new directions. Indeed, recent works by the author, Andrews, Eguchi, Hikami, Kac, Lawrence, Malmendier, Mellit, Okada, Wakimoto, and Zagier $[15,18,21,26,92,120,121,122,130,131,144,152,153,211]$ apply this theory to:

- Donaldson invariants

- Gauge theory

- Representation theory of Lie superalgebras

- Knot theory

- Mathematical physics

- Probability theory

- Topology.

The author and his collaborators have aimed to employ this new perspective to answer deep questions about many of the number theoretic topics captured by the web of Ramanujan's mock theta functions. Here we describe the implications of this theory to:

- Partitions and $q$-series

- Modular forms

- Traces of singular moduli

- Borcherds products

- Modular L-functions à la Kohnen-Waldspurger and Kohnen-Zagier.

REMARK 3. This paper is not intended to be a comprehensive treatise; instead, it is only meant to serve as an overview of the main aspects of this program.

The remainder of the paper is organized as follows. In Section 6, we give one beautiful example of Zwegers work, as it pertains to Ramanujan's mock theta functions $f(q)$ and $\omega(q)$, and we briefly describe his important construction of a weight 1/2 non-holomorphic Jacobi form. In Section 7 we give the formal definition of a harmonic Maass form, and we present many of their basic properties. In Section 8 we give various examples of such forms. In the remaining sections we summarize our results on the following topics:

Section 9 (Dyson-Ramanujan theory of partition congruences).

In an effort to provide a combinatorial explanation of Ramanujan's congruences

$$
\begin{aligned}
p(5 n+4) & \equiv 0 \quad(\bmod 5) \\
p(7 n+5) & \equiv 0 \quad(\bmod 7), \\
p(11 n+6) & \equiv 0 \quad(\bmod 11)
\end{aligned}
$$


Dyson introduced [90] the so-called rank of a partition, a delightfully simple statistic. The rank of a partition is defined to be its largest part minus the number of its parts.

For example, the table below includes the ranks of the partitions of 4 .

\begin{tabular}{|c|c|c|}
\hline Partition & $\overline{\text { Rank }}$ & Rank $\bmod 5$ \\
\hline 4 & $4-1=3$ & 3 \\
\hline $3+1$ & $3-2=1$ & 1 \\
\hline $2+2$ & $2-2=0$ & 0 \\
\hline $2+1+1$ & $2-3=-1$ & 4 \\
\hline $1+1+1+1$ & $1-4=-3$ & 2 \\
\hline
\end{tabular}

One observes that each residue class modulo 5 occurs exactly once in the last column above. Based on further numerics, Dyson made the following conjecture whose truth provides a combinatorial explanation of Ramanujan's congruences modulo 5 and 7 .

Conjecture (1944, Dyson). The partitions of $5 n+4$ (resp. $7 n+5)$ form 5 (resp. 7) groups of equal size when sorted by their ranks modulo 5 (resp. 7).

In 1954, Atkin and H. P. F. Swinnerton-Dyer proved [31] Dyson's conjecture. ${ }^{11}$ In view of the more general theory of partition congruences, which includes examples such as

$$
p(4063467631 n+30064597) \equiv 0 \quad(\bmod 31),
$$

it is natural to investigate the role that Dyson's rank plays in the general theory of partition congruences. Using a new class of harmonic Maass forms, which will be described in Section 8.4, the author and K. Bringmann $[\mathbf{4 7}, \mathbf{5 5}]$ have obtained general results in this direction. These works will be described in Section 9.

Section 10 (Eulerian series as modular forms).

The Rogers-Ramanujan identities

$$
\begin{aligned}
& \prod_{n=0}^{\infty} \frac{1}{\left(1-q^{5 n+1}\right)\left(1-q^{5 n+4}\right)}=1+\sum_{n=1}^{\infty} \frac{q^{n^{2}}}{(1-q)\left(1-q^{2}\right) \cdots\left(1-q^{n}\right)}, \\
& \prod_{n=0}^{\infty} \frac{1}{\left(1-q^{5 n+2}\right)\left(1-q^{5 n+3}\right)}=1+\sum_{n=1}^{\infty} \frac{q^{n^{2}+n}}{(1-q)\left(1-q^{2}\right) \cdots\left(1-q^{n}\right)},
\end{aligned}
$$

and the mock theta conjectures provide examples of Eulerian series as modular forms. Thanks to the framework of the theory of harmonic Maass forms, we have a better understanding of the modular transformation properties of certain Eulerian series. In Section 10 we discuss work by the author,

\footnotetext{
${ }^{11} \mathrm{~A}$ short calculation reveals that the obvious generalization of the conjecture cannot hold for 11 .
} 
Bringmann, and R. Rhoades [57] on Eulerian series as modular forms. This work immediately gives many new identities such as

$$
\sum_{n \geq 0} \frac{q^{n(n+1)}\left(-q^{2} ; q^{2}\right)_{n}}{\left(q ; q^{2}\right)_{n+1}^{2}}+\sum_{n \geq 0} \frac{q^{n(n+1)}\left(-q^{2} ; q^{2}\right)_{n}}{\left(-q ; q^{2}\right)_{n+1}^{2}}=2 \frac{\left(q^{4} ; q^{4}\right)_{\infty}^{5}}{\left(q^{2} ; q^{2}\right)_{\infty}^{4}} .
$$

Section 11 (Exact formulas).

As described earlier, Rademacher perfected the Hardy-Ramanujan asymptotic (2.2) to obtain an exact formula for $p(n)$ (for example, see $[168,169])$. To state his formula, let $I_{s}(x)$ be the usual $I$-Bessel function of order $s$, and let $e(x):=e^{2 \pi i x}$. Furthermore, if $k \geq 1$ and $n$ are integers, then let

$$
A_{k}(n):=\frac{1}{2} \sqrt{\frac{k}{12}} \sum_{\substack{x \\ x^{2} \equiv-24 n+1 \quad(\bmod 24 k)}} \chi_{12}(x) \cdot e\left(\frac{x}{12 k}\right),
$$

where the sum runs over the residue classes modulo $24 k$, and where

$$
\chi_{12}(x):=\left(\frac{12}{x}\right)
$$

If $n$ is a positive integer, then one version of Rademacher's formula reads

$$
p(n)=2 \pi(24 n-1)^{-\frac{3}{4}} \sum_{k=1}^{\infty} \frac{A_{k}(n)}{k} \cdot I_{\frac{3}{2}}\left(\frac{\pi \sqrt{24 n-1}}{6 k}\right) .
$$

We address the following classical partition problem.

Problem. Determine exact formulas for $N_{e}(n)$ (resp. $\left.N_{o}(n)\right)$, the number of partitions of $n$ with even (resp. odd) Dyson rank.

Thanks to Rademacher's formula, and the obvious fact that

$$
p(n)=N_{e}(n)+N_{o}(n),
$$

it turns out that this question is equivalent to the problem of deriving exact formulas for the coefficients

$$
\alpha(n)=N_{e}(n)-N_{o}(n)
$$

of the mock theta function $f(q)$. This deduction is a simple modification of the proof of Theorem 2.2.

As mentioned earlier, Andrews and Dragonette had already proved that

$$
\alpha(n) \sim \frac{(-1)^{n-1}}{2 \sqrt{n-\frac{1}{24}}} \cdot e^{\pi \sqrt{\frac{n}{6}-\frac{1}{144}}} .
$$

This result falls short of the problem of obtaining an exact formula, and as a consequence represents the obstruction to obtaining formulas for $N_{e}(n)$ and $N_{o}(n)$. In his plenary address "Partitions: At the interface of $q$-series and modular forms", delivered at the Millenial Number Theory Conference at the University of Illinois in 2000, Andrews highlighted this classical problem 
by promoting his conjecture ${ }^{12}$ of 1966 (see page 456 of [7], and Section 5 of [17]) for the coefficients $\alpha(n)$.

Conjecture. (Andrews-Dragonette)

If $n$ is a positive integer, then

$\alpha(n)=\pi(24 n-1)^{-\frac{1}{4}} \sum_{k=1}^{\infty} \frac{(-1)^{\left\lfloor\frac{k+1}{2}\right\rfloor} A_{2 k}\left(n-\frac{k\left(1+(-1)^{k}\right)}{4}\right)}{k} \cdot I_{\frac{1}{2}}\left(\frac{\pi \sqrt{24 n-1}}{12 k}\right)$.

The author and Bringmann [53] proved this conjecture using work of Zwegers and the theory of Maass-Poincaré series. Since $N_{e}(n)=(p(n)+$ $\alpha(n)) / 2$ and $N_{o}(n)=(p(n)-\alpha(n)) / 2$, the proof of the conjecture, combined with Rademacher's exact formula, provides the desired formulas for $N_{e}(n)$ and $N_{o}(n)$.

The proof of the Andrews-Dragonette Conjecture is a special case of a more general problem.

Problem. Determine exact formulas for the coefficients of holomorphic parts of harmonic Maass forms.

We shall also give general theorems which provide such exact formulas for harmonic Maass forms with weight $\leq 1 / 2$. These new results include the classic results of Rademacher and Zuckerman $[169,170,216,217]$ which give exact formulas for the Fourier coefficients of negative weight weakly holomorphic modular forms. Indeed, these results follow since weakly holomorphic modular forms are harmonic Maass forms. They also recover some results of Bruinier, Hejhal, and Niebur $[62,117,160,161]$ for harmonic Maass forms of non-positive weight.

Section 12 (Applications to classical modular forms).

Using the properties of various differential operators, it is possible to address old problems about classical modular forms. For example, we address the classification of linear relations among cuspidal Poincaré series, and we obtain a theorem which detects the vanishing of Hecke eigenvalues for integer weight newforms. We also show that Lehmer's Conjecture, which asserts that none of the coefficients of the discriminant function

$$
\Delta(z)=\sum_{n=1}^{\infty} \tau(n) q^{n}:=q \prod_{n=1}^{\infty}\left(1-q^{n}\right)^{24}=q-24 q+252 q^{3}-\cdots
$$

vanish, is implied by the irrationality of the coefficients of the "holomorphic part" of a certain Maass-Poincaré series.

The work on linear relations among cuspidal Poincaré series is contained in Rhoades's Ph.D. thesis [179] (also see [178]). The theorem which detects vanishing Fourier coefficients is in a recent paper by J. H. Bruinier, Rhoades, and the author [68]. These results are described in Section 12.

\footnotetext{
${ }^{12}$ This conjecture is suggested as a speculation by Dragonette in [85].
} 
Section 13 (Generating functions for singular moduli).

Let $j(z)$ be the usual modular function for $\mathrm{SL}_{2}(\mathbb{Z})$

$$
j(z)=q^{-1}+744+196884 q+21493760 q^{2}+\cdots .
$$

The values of modular functions such as $j(z)$ at imaginary quadratic arguments in $\mathbb{H}$ are known as singular moduli. Singular moduli are algebraic integers which play many roles in number theory. For example, they generate class fields of imaginary quadratic fields, and they parameterize isomorphism classes of elliptic curves with complex multiplication.

In an important paper [212], Zagier gave a new proof of Borcherds' famous theorem on the infinite product expansions of integer weight modular forms on $\mathrm{SL}_{2}(\mathbb{Z})$ with Heegner divisor. This proof, as well as all of the results of $[\mathbf{2 1 2}]$, are connected to his beautiful observation that the generating functions for traces of singular moduli are essentially weight $3 / 2$ weakly holomorphic modular forms.

Zagier's paper has inspired an extraordinary number of research papers with generalizations in a variety of directions in works by the author, Bringmann, Bruinier, D. Choi, W. Duke, A. Folsom, J. Funke, O. Imamoḡlu, P. Jenkins, D. Jeon, S.-Y. Kang, C. Kim, R. Masri, A. Miller, A. Pixton, J. Rouse, and A. Toth $[54,58,64,65,77,78,87,88,89,95,154]$. In Section 13 we describe one theorem which gives typical examples of such generating functions. In addition to giving further generating functions for singular moduli, we shall also describe a beautiful theorem of Duke $[\mathbf{8 7}]$ related to the classical observation that

$$
e^{\pi \sqrt{163}}=262537412640768743.9999999999992 \ldots
$$

is nearly an integer.

Section 14 (Borcherds Products).

Recently, Borcherds $[\mathbf{4 2}, \mathbf{4 3}, 44]$ provided a striking description for the exponents in the naive infinite product expansion of many modular forms, those forms with a Heegner divisor. He proved that the exponents in these infinite product expansions are certain coefficients of modular forms of weight $1 / 2$. For example, let $c(n)$ denote the integer exponents one obtains by expressing the classical Eisenstein series $E_{4}(z)$ as an infinite product:

$E_{4}(z)=1+240 \sum_{n=1}^{\infty} \sigma_{3}(n) q^{n}=(1-q)^{-240}\left(1-q^{2}\right)^{26760} \cdots=\prod_{n=1}^{\infty}\left(1-q^{n}\right)^{c(n)}$.

Borcherds proved that there is a weight 1/2 meromorphic modular form

$$
G(z)=\sum_{n \geq-3} b(n) q^{n}=q^{-3}+4-240 q+26760 q^{4}+\cdots-4096240 q^{9}+\ldots
$$

with the property that $c(n)=b\left(n^{2}\right)$ for every positive integer $n$.

These results may be generalized where the Borcherds exponents are given by certain Fourier coefficients of weight $1 / 2$ harmonic Maass forms. 
As a nice example, consider Ramanujan's mock theta function (see (3.1))

$$
\begin{aligned}
\omega(q) & =\sum_{n=0}^{\infty} \frac{q^{2 n^{2}+2 n}}{\left(q ; q^{2}\right)_{n+1}^{2}} \\
& =\frac{1}{(1-q)^{2}}+\frac{q^{4}}{(1-q)^{2}\left(1-q^{3}\right)^{2}}+\frac{q^{12}}{(1-q)^{2}\left(1-q^{3}\right)^{2}\left(1-q^{5}\right)^{2}}+\cdots .
\end{aligned}
$$

Define integers $a(n)$ by the expression

$$
\begin{aligned}
\sum_{n \in \mathbb{Z}+1 / 3} a(n) q^{n} & :=-2 q^{1 / 3}\left(\omega\left(q^{1 / 2}\right)+\omega\left(-q^{1 / 2}\right)\right) \\
& =-4 q^{1 / 3}-12 q^{4 / 3}-24 q^{7 / 3}-40 q^{10 / 3}-\ldots
\end{aligned}
$$

These new results on generalized Borcherds products imply that

$$
\begin{aligned}
& \prod_{n=1}^{\infty}\left(\frac{1+\sqrt{-2} q^{n}-q^{2 n}}{1-\sqrt{-2} q^{n}-q^{2 n}}\right)^{\left(\frac{n}{3}\right) a\left(n^{2} / 3\right)}=1-8 \sqrt{-2} q-(64-24 \sqrt{-2}) q^{2} \\
& \quad+(384+168 \sqrt{-2}) q^{3}+(64-1768 \sqrt{-2}) q^{4}+\ldots
\end{aligned}
$$

is a modular form on the congruence subgroup $\Gamma_{0}(6)$.

A brief indication of these results is given in Section 14 .

Section 15 (Derivatives and values of $L$-functions).

Once armed with a generalized Borcherds-type theorem, one is then able to construct modular forms with a prescribed divisor, a twisted Heegner divisor, with the additional property that one can determine the field of definition of the product Fourier expansion. For example, the modular form in (5.4) clearly has Fourier coefficients in $\mathbb{Z}[\sqrt{-2}]$, thanks to the fact that Ramanujan's mock theta function $\omega(q)$ has integer coefficients. This phenomenon is extremely useful in arithmetic geometry since it allows us to determine whether certain divisors vanish in the Jacobian of a modular curve, a condition which plays a central role in the work of Gross and Zagier on the Birch and Swinnerton-Dyer Conjecture. Here we explain how to make use of these generalized Borcherds products to extend deep theorems of Waldspurger and Kohnen.

In the 1980s, Waldspurger [205], and Kohnen and Zagier [135, 136, 137] established that certain half-integral weight modular forms serve as generating functions of a new type. Using the Shimura correspondence [192], they proved that certain coefficients of half-integral weight cusp forms essentially are square-roots of central values of quadratic twists of modular $L$-functions.

When the weight is $3 / 2$, these results appear prominently in works related to the Birch and Swinnerton-Dyer Conjecture. For example, Tunnell [202] made great use of explicit examples of these results in his work on the ancient "congruent number problem": the determination of those positive integers which are areas of right triangles with rational sidelengths. More generally, these results of Kohnen, Waldspurger and Zagier play central roles 
in the deep works of Gross, Zagier and Kohnen $[\mathbf{1 0 8 , 1 0 7 ]}$ on the Birch and Swinnerton-Dyer Conjecture.

The author and Bruinier [67] have generalized this theorem of Waldspurger and Kohnen to prove that the Fourier coefficients of weight $1 / 2$ harmonic Maass forms encode the vanishing and nonvanishing of both the central values and derivatives of quadratic twists of weight 2 modular $L$-functions.

Here we describe a special case of the main theorem. Suppose that

$$
G(z)=\sum_{n=1}^{\infty} B_{G}(n) q^{n} \in S_{2}(p)
$$

is a weight 2 newform with prime level $p$. As usual, we let

$$
L(G, s)=\sum_{n=1}^{\infty} \frac{B_{G}(n)}{n^{s}}
$$

denote its Hecke $L$-function. If $\Delta$ is a fundamental discriminant of a quadratic field coprime to $p$, then we let $L\left(G, \chi_{\Delta}, s\right)$ be the quadratic twist Hecke $L$-function

$$
L\left(G, \chi_{\Delta}, s\right)=\sum_{n=1}^{\infty} \frac{B_{G}(n) \chi_{\Delta}(n)}{n^{s}},
$$

where $\chi_{\Delta}(\bullet):=(\underline{\bullet})$ denotes the Kronecker character for $\mathbb{Q}(\sqrt{\Delta})$. It is well known that $L(G, s)$ and $L\left(G, \chi_{\Delta}, s\right)$ have functional equations relating their values at $s$ and $2-s$.

Here is a special case of the main result in [67].

TheOREM 5.1. Assume the hypotheses and notation above. In addition, suppose that the sign of the functional equation of $L(G, s)$ is $\epsilon(G)=-1$. Then there is a weight 1/2 harmonic Maass form $f(z)$ on $\Gamma_{0}(4 p)$ with Fourier expansion

$$
f(z)=\sum_{n \gg-\infty} c_{g}^{+}(n) q^{n}+\sum_{n<0} c_{g}^{-}(n) \Gamma(1 / 2 ; 4 \pi|n| y) q^{n},
$$

where $y=\operatorname{Im}(z)$ and $\Gamma(\alpha ; t)$ is the usual incomplete Gamma-function (see (7.5)), satisfying the following:

(1) If $\Delta<0$ is a fundamental discriminant for which $\left(\frac{\Delta}{p}\right)=1$, then

$$
L\left(G, \chi_{\Delta}, 1\right)=\alpha_{G} \cdot \sqrt{|\Delta|} \cdot c_{g}^{-}(\Delta)^{2},
$$

where $\alpha_{G}$ is an explicit non-zero constant.

(2) If $\Delta>0$ is a fundamental discriminant for which $\left(\frac{\Delta}{p}\right)=1$, then $L^{\prime}\left(G, \chi_{\Delta}, 1\right)=0$ if and only if $c_{g}^{+}(\Delta)$ is algebraic.

REMARK 4. Theorem 5.1 is a special case of the general result which holds for all levels, and any arbitrary sign. 
EXAMPLE 5.2. Here we present an example which numerically illustrates the most general form of Theorem 5.1 for the weight 2 newform $G$ which corresponds to the conductor 37 elliptic curve

$$
E: y^{2}=x^{3}+10 x^{2}-20 x+8 .
$$

The table below includes some of the coefficients of a suitable $f$ which were numerically computed by F. Strömberg (also see [69]).

\begin{tabular}{ccc}
\hline \hline$\Delta$ & $c_{g}^{+}(-\Delta)$ & $L^{\prime}(E(\Delta), 1)=L^{\prime}\left(G, \chi_{\Delta}, 1\right)$ \\
\hline & $1.0267149116 \ldots$ & $1.4792994920 \ldots$ \\
-3 & $1.2205364009 \ldots$ & $1.8129978972 \ldots$ \\
-4 & $1.6900297463 \ldots$ & $2.1107189801 \ldots$ \\
-7 & $\vdots$ & $\vdots$ \\
-136 & $-4.8392675993 \ldots$ & $5.7382407649 \ldots$ \\
-139 & -6 & 0 \\
-151 & $-0.8313568817 \ldots$ & $6.6975085515 \ldots$ \\
$\vdots$ & $\vdots$ & $\vdots$ \\
-815 & $121.1944103120 \ldots$ & $4.7492583693 \ldots$ \\
-823 & 312 & 0 \\
\hline
\end{tabular}

Strictly speaking, the cases where $\Delta=-139$ and -823 were not obtained numerically. We have that $L^{\prime}(E(-139), 1)=L^{\prime}(E(-823), 1)=0$ by the Gross-Zagier formula. The evaluations $c_{g}^{+}(139)=-6$ and $c_{g}^{+}(823)=312$ arise from explicit generalized Borcherds products (for example, see Example 8.3 of $[\mathbf{6 7}])$. The rank 3 elliptic curve $E(-139)$ is quite famous, for it was used as input data for Goldfeld's celebrated effective solution to Gauss's "Class Number Problem". For the other $\Delta$ in the table, the derivatives are non-vanishing and the coefficients $c_{g}^{+}(-\Delta)$ are transcendental.

Theorem 5.1 relates the algebraicity of coefficients of harmonic Maass forms to the vanishing of derivatives of modular $L$-functions. It is then natural to ask whether these ideas can be used to exactly compute these derivatives. In other words, can the theory of harmonic Maass forms be used to obtain the deep formulas (as well as generalizations) of Gross and Zagier which relate heights of Heegner points to such derivatives? Bruinier and T. Yang have used these results, combined with their theory of Green's functions and theta lifts for harmonic Maass forms, to show that this is indeed the case. In addition to formulating a deep conjecture about derivatives of $L$-functions and heights (see Section 15.2), they have proved the following striking theorem (see $[\mathbf{7 0}]$ ).

THEOREM 5.3. If $G \in S_{2}(N)$ is a weight 2 newform with the property that the sign of the functional equation of $L(G, s)$ is $\epsilon(G)=-1$, then there 
is a weight 1/2 harmonic Maass form $f$, a weight 3/2 cusp form $g$, and a Heegner divisor $Z(f)$ whose Neron-Tate height pairing is given by

$$
\langle Z(f), Z(f)\rangle_{N T}=\frac{2 \sqrt{N}}{\pi\|g\|^{2}} L^{\prime}(G, 1) .
$$

REMARK 5. To ease notation in Theorem 5.3, we did not describe the relationship between $G, g, f$ and $Z(f)$. Loosely speaking, they are related as follows. We let $g$ be a weight $3 / 2$ cusp form whose image under the Shimura correspondence is $G$. The harmonic Maass form $f$ in Theorems 5.1 and 5.3 is selected so that its principal part is defined over the number field generated by the coefficients of $G$, and also satisfies

$$
\xi_{\frac{1}{2}}(f)=\|g\|^{-2} g \text {. }
$$

Here $\xi_{\frac{1}{2}}$ is the differential operator considered in Lemma 7.4. The Heegner divisor $Z(f)$ is then defined using the principal part of $f$.

These works shall be described in Section 15 .

\section{The mock theta functions of Ramanujan d'après Zwegers}

Here we give a typical example of Zwegers's results on Ramanujan's mock theta functions, and we briefly describe his construction of a weight 1/2 non-holomorphic Jacobi form.

6.1. Ramanujan's mock theta functions $f(q)$ and $\boldsymbol{\omega}(q)$. We first consider Ramanujan's third order mock theta function $f(q)$. Important results concerning its modularity properties were first obtained by G. N. Watson in [206]. Although $f(q)$ is not the Fourier expansion of a usual meromorphic modular form, in this classic paper Watson determined its complicated modular transformation properties. Watson's modular transformation formulas are difficult to grasp at first glance; they involve another third order mock theta function, as well as terms arising from Mordell integrals.

Zwegers [218] nicely packaged Watson's results in terms of real analytic vector-valued modular forms. We now describe this result. As before, let $\omega(q)$ be the third order mock theta function defined by (3.1). Define the vector-valued function $F(z)$ by

$$
F(z)=\left(F_{0}(z), F_{1}(z), F_{2}(z)\right)^{T}:=\left(q^{-\frac{1}{24}} f(q), 2 q^{\frac{1}{3}} \omega\left(q^{\frac{1}{2}}\right), 2 q^{\frac{1}{3}} \omega\left(-q^{\frac{1}{2}}\right)\right)^{T} .
$$

Similarly, let $G(z)$ be the vector-valued non-holomorphic function defined by

$$
G(z)=\left(G_{0}(z), G_{1}(z), G_{2}(z)\right)^{T}:=2 i \sqrt{3} \int_{-\bar{z}}^{i \infty} \frac{\left(g_{1}(\tau), g_{0}(\tau),-g_{2}(\tau)\right)^{T}}{\sqrt{-i(\tau+z)}} d \tau
$$


where the $g_{i}(\tau)$ are the cuspidal weight $3 / 2$ theta functions

$$
\begin{aligned}
g_{0}(\tau) & :=\sum_{n=-\infty}^{\infty}(-1)^{n}\left(n+\frac{1}{3}\right) e^{3 \pi i\left(n+\frac{1}{3}\right)^{2} \tau}, \\
g_{1}(\tau) & :=-\sum_{n=-\infty}^{\infty}\left(n+\frac{1}{6}\right) e^{3 \pi i\left(n+\frac{1}{6}\right)^{2} \tau} \\
g_{2}(\tau) & :=\sum_{n=-\infty}^{\infty}\left(n+\frac{1}{3}\right) e^{3 \pi i\left(n+\frac{1}{3}\right)^{2} \tau} .
\end{aligned}
$$

Using these vector-valued functions, Zwegers defined the vector-valued function $H(z)$ by

$$
H(z):=F(z)-G(z)
$$

The following description of $H(z)$ is the main result of $[\mathbf{2 1 8}]$.

THEOREM 6.1. (Zwegers)

The function $H(z)$ is a vector-valued real analytic modular form of weight $1 / 2$ satisfying

$$
\begin{aligned}
& H(z+1)=\left(\begin{array}{ccc}
\zeta_{24}^{-1} & 0 & 0 \\
0 & 0 & \zeta_{3} \\
0 & \zeta_{3} & 0
\end{array}\right) H(z) \\
& H(-1 / z)=\sqrt{-i z} \cdot\left(\begin{array}{ccc}
0 & 1 & 0 \\
1 & 0 & 0 \\
0 & 0 & -1
\end{array}\right) H(z),
\end{aligned}
$$

where $\zeta_{n}:=e^{2 \pi i / n}$. Furthermore, $H(z)$ is an eigenfunction of the Casimir operator $\Omega_{\frac{1}{2}}:=-4 y^{2} \frac{\partial^{2}}{\partial z \partial \bar{z}}+i y \frac{\partial}{\partial \bar{z}}+\frac{3}{16}$ with eigenvalue $\frac{3}{16}$, where $z=x+i y$, $\frac{\partial}{\partial z}=\frac{1}{2}\left(\frac{\partial}{\partial x}-i \frac{\partial}{\partial y}\right)$, and $\frac{\partial}{\partial \bar{z}}=\frac{1}{2}\left(\frac{\partial}{\partial x}+i \frac{\partial}{\partial y}\right)$.

Remark 6. This Casimir operator is essentially the weight $1 / 2$ hyperbolic Laplacian operator (see (7.1)) which plays an important role in the definition of harmonic Maass forms.

This beautiful theorem nicely describes the modular transformations of both $f(q)$ and $\omega(q)$. In particular, they are essentially the "holomorphic parts" of components of a 3 -dimensional vector-valued weight $1 / 2$ real analytic modular form. The "non-holomorphic" parts of these components are "period integrals" of classical weight $3 / 2$ functions which turn out to be single variable theta functions. The functions in Theorem 6.1 can be thought of as prototypes for the theory of harmonic Maass forms. 
6.2. Zwegers's weight $1 / 2$ non-holomorphic Jacobi form. In his thesis, Zwegers constructed weight $1 / 2$ harmonic Maass forms by making use of the transformation properties of Lerch sums. Here we briefly recall some of these important results which address the difficult problem of constructing weight 1/2 harmonic Maass forms.

For $\tau \in \mathbb{H}, u, v \in \mathbb{C} \backslash(\mathbb{Z} \tau+\mathbb{Z})$, Zwegers defined the function

$$
\mu(u, v ; \tau):=\frac{z^{1 / 2}}{\vartheta(v ; \tau)} \cdot \sum_{n \in \mathbb{Z}} \frac{(-w)^{n} q^{n(n+1) / 2}}{1-z q^{n}},
$$

where $z:=e^{2 \pi i u}, w:=e^{2 \pi i v}, q:=e^{2 \pi i \tau}$ and

$$
\vartheta(v ; \tau):=\sum_{\nu \in \mathbb{Z}+\frac{1}{2}} e^{\pi i \nu} w^{\nu} q^{\nu^{2} / 2} .
$$

REMARK 7 . We stress that $q:=e^{2 \pi i \tau}$ in this subsection, which is a brief departure from our convention that $q:=e^{2 \pi i z}$.

Zwegers (see Section 1.3 of $[\mathbf{2 1 9}]$ ) proves that $\mu(u, v ; \tau)$ satisfies the following important properties.

Lemma 6.2. Assuming the notation above, we have that

$$
\begin{aligned}
\mu(u, v ; \tau) & =\mu(v, u ; \tau), \\
\mu(u+1, v ; \tau) & =-\mu(u, v ; \tau), \\
z^{-1} w q^{-\frac{1}{2}} \mu(u+\tau, v ; \tau) & =-\mu(u, v ; \tau)-i z^{-\frac{1}{2}} w^{\frac{1}{2}} q^{-\frac{1}{8}}, \\
\mu(u, v ; \tau+1) & =\zeta_{8}^{-1} \mu(u, v ; \tau) \quad\left(\zeta_{N}:=e^{2 \pi i / N}\right) \\
(\tau / i)^{-\frac{1}{2}} e^{\pi i(u-v)^{2} / \tau} \mu\left(\frac{u}{\tau}, \frac{v}{\tau} ;-\frac{1}{\tau}\right) & =-\mu(u, v ; \tau)+\frac{1}{2 i} h(u-v ; \tau),
\end{aligned}
$$

where

$$
h(z ; \tau):=\int_{-\infty}^{\infty} \frac{e^{\pi i x^{2} \tau-2 \pi x z} d x}{\cosh \pi x} .
$$

REMARK 8. The integral $h(z ; \tau)$ is known as a Mordell integral.

Lemma 6.2 shows that $\mu(u, v ; \tau)$ is nearly a weight $1 / 2$ Jacobi form, where $\tau$ is the modular variable. Zwegers then uses $\mu$ to construct weight $1 / 2$ harmonic Maass forms. He achieves this by modifying $\mu$ to obtain a function $\widehat{\mu}$ which he then uses as building blocks for such Maass forms. To make this precise, for $\tau \in \mathbb{H}$ and $u \in \mathbb{C}$, let $c:=\operatorname{Im}(u) / \operatorname{Im}(\tau)$, and let

$$
R(u ; \tau):=\sum_{\nu \in \mathbb{Z}+\frac{1}{2}}(-1)^{\nu-\frac{1}{2}}\{\operatorname{sgn}(\nu)-E((\nu+c) \sqrt{2 \operatorname{Im}(\tau)})\} e^{-2 \pi i \nu u} q^{-\nu^{2} / 2},
$$

where $E(x)$ is the odd function

$$
E(x):=2 \int_{0}^{x} e^{-\pi u^{2}} d u=\operatorname{sgn}(x)\left(1-\beta\left(x^{2}\right)\right),
$$


where for positive real $x$ we let $\beta(x):=\int_{x}^{\infty} u^{-\frac{1}{2}} e^{-\pi u} d u$.

Using $\mu$ and $R$, Zwegers defines the real analytic function

$$
\widehat{\mu}(u, v ; \tau):=\mu(u, v ; \tau)+\frac{i}{2} R(u-v ; \tau) .
$$

Zwegers construction of weight 1/2 harmonic Maass forms depends on the following theorem (see Section 1.4 of $[\mathbf{2 1 9}]$ ).

THEOREM 6.3. Assuming the notation and hypotheses above, we have that

$$
\begin{aligned}
\widehat{\mu}(u, v ; \tau) & =\widehat{\mu}(v, u ; \tau), \\
\widehat{\mu}(u+1, v ; \tau) & =z^{-1} w q^{-\frac{1}{2}} \widehat{\mu}(u+\tau, v ; \tau)=-\widehat{\mu}(u, v ; \tau), \\
\zeta_{8} \widehat{\mu}(u, v ; \tau+1) & =-(\tau / i)^{-\frac{1}{2}} e^{\pi i(u-v)^{2} / \tau} \widehat{\mu}\left(\frac{u}{\tau}, \frac{v}{\tau} ;-\frac{1}{\tau}\right)=\widehat{\mu}(u, v ; \tau) .
\end{aligned}
$$

Moreover, if $A=\left(\begin{array}{ll}\alpha & \beta \\ \gamma & \delta\end{array}\right) \in \mathrm{SL}_{2}(\mathbb{Z})$, then

$\widehat{\mu}\left(\frac{u}{\gamma \tau+\delta}, \frac{v}{\gamma \tau+\delta} ; \frac{\alpha \tau+\beta}{\gamma \tau+\delta}\right)=\chi(A)^{-3}(\gamma \tau+\delta)^{\frac{1}{2}} e^{-\pi i \gamma(u-v)^{2} /(\gamma \tau+\delta)} \cdot \widehat{\mu}(u, v ; \tau)$,

where $\chi(A):=\eta(A \tau) /\left((\gamma \tau+\delta)^{\frac{1}{2}} \eta(\tau)\right)$.

Theorem 6.3 shows that $\widehat{\mu}(u, v ; \tau)$ is essentially a weight $1 / 2$ non-holomorphic Jacobi form. In analogy with the classical theory of Jacobi forms, one may then obtain harmonic Maass forms by making suitable specializations for $u$ and $v$ by elements in $\mathbb{Q} \tau+\mathbb{Q}$, and by multiplying by appropriate powers of $q$. Without this result, it would be very difficult to explicitly construct examples of weight 1/2 harmonic Maass forms.

Harmonic Maass forms of weight $k$ are mapped to classical modular forms (see Lemma 7.4), their so-called shadows, by the differential operator

$$
\xi_{k}:=2 i y^{k} \cdot \frac{\bar{\partial}}{\partial \bar{\tau}}
$$

The following lemma makes it clear that the shadows of the real analytic forms arising from $\widehat{\mu}$ can be described in terms of weight $3 / 2$ theta functions.

LEMma 6.4. [Lemma 1.8 of [219]] The function $R$ is real analytic and satisfies

$$
\frac{\partial R}{\partial \bar{u}}(u ; \tau)=\sqrt{2} y^{-\frac{1}{2}} e^{-2 \pi c^{2} y} \vartheta(\bar{u} ;-\bar{\tau}),
$$

where $c:=\operatorname{Im}(u) / \operatorname{Im}(\tau)$. Moreover, we have that

$$
\frac{\partial}{\partial \bar{\tau}} R(a \tau-b ; \tau)=-\frac{i}{\sqrt{2 y}} e^{-2 \pi a^{2} y} \sum_{\nu \in \mathbb{Z}+\frac{1}{2}}(-1)^{\nu-\frac{1}{2}}(\nu+a) e^{-\pi i \nu^{2} \bar{\tau}-2 \pi i \nu(a \bar{\tau}-b)} .
$$




\section{Harmonic Maass forms}

For the remainder of the paper, we shall assume that the reader is familiar with the classical theory of elliptic modular forms (for example, see $[71,84,125,134,143,155,164,177,185,193,196])$.

D. Niebur $[\mathbf{1 6 0}, \mathbf{1 6 1}]$ and D. Hejhal $[\mathbf{1 1 7}]$ constructed certain nonholomorphic Poincaré series which turn out to be examples of harmonic Maass forms. Bruinier [61] made great use of these Poincaré series in his early work on Borcherds lifts and Green's functions. He then realized the importance of developing a "theory of harmonic Maass forms" in its own right. Later in joint work with Funke [63], he developed the fundamental results of this theory, some of which we describe here. After making the necessary definitions, we shall discuss Hecke operators and various differential operators. The interplay between harmonic Maass forms and classical modular forms shall play an important role throughout this paper.

7.1. Definitions. In 1949, H. Maass introduced the notion of a Maass form $^{13}$ (see $\left.[\mathbf{1 4 9}, \mathbf{1 5 0}]\right)$. He constructed these non-holomorphic automorphic forms using Hecke characters of real quadratic fields, in analogy with Hecke's theory [115] of modular forms with complex multiplication (see [180] for a modern treatment).

To define these functions, let $\Delta=\Delta_{0}$ be the hyperbolic Laplacian

$$
\Delta:=-y^{2}\left(\frac{\partial^{2}}{\partial x^{2}}+\frac{\partial^{2}}{\partial y^{2}}\right)
$$

where $z=x+i y \in \mathbb{H}$ with $x, y \in \mathbb{R}$. It is a second-order differential operator which acts on functions on $\mathbb{H}$, and it is invariant under the action of $\mathrm{SL}_{2}(\mathbb{R})$.

A Maass form for a subgroup $\Gamma \subset \mathrm{SL}_{2}(\mathbb{Z})$ is a smooth function $f: \mathbb{H} \rightarrow \mathbb{C}$ satisfying:

(1) For every $\left(\begin{array}{ll}a & b \\ c & d\end{array}\right) \in \Gamma$, we have

$$
f\left(\frac{a z+b}{c z+d}\right)=f(z) .
$$

(2) We have that $f$ is an eigenfunction of $\Delta$.

(3) There is some $N>0$ such that

$$
f(x+i y)=O\left(y^{N}\right)
$$

as $y \rightarrow+\infty$.

Furthermore, we call $f$ a Maass cusp form if

$$
\int_{0}^{1} f(z+x) d x=0 .
$$

There is now a vast literature on Maass forms thanks to the works of many authors such as Hejhal, Iwaniec, Maass, Roelcke, Selberg, Terras, Venkov,

\footnotetext{
${ }^{13}$ In analogy with the eigenvalue problem for the vibrating membrane, Maass referred to these automorphic forms as Wellenformen, or waveforms.
} 
among many others (for example, see $[116,117, \mathbf{1 2 4}, \mathbf{1 2 6}, \mathbf{1 4 9}, \mathbf{1 5 0}, \mathbf{1 8 1}$, 191, 199, 200, 203]).

This paper concerns a generalization of this notion of Maass form. Following Bruinier and Funke [63], we define the notion of a harmonic Maass form of weight $k \in \frac{1}{2} \mathbb{Z}$ as follows. As before, we let $z=x+i y \in \mathbb{H}$ with $x, y \in \mathbb{R}$. We define the weight $k$ hyperbolic Laplacian $\Delta_{k}$ by

$$
\Delta_{k}:=-y^{2}\left(\frac{\partial^{2}}{\partial x^{2}}+\frac{\partial^{2}}{\partial y^{2}}\right)+i k y\left(\frac{\partial}{\partial x}+i \frac{\partial}{\partial y}\right) .
$$

For odd integers $d$, define $\epsilon_{d}$ by

$$
\epsilon_{d}:=\left\{\begin{array}{lll}
1 & \text { if } d \equiv 1 & (\bmod 4) \\
i & \text { if } d \equiv 3 & (\bmod 4)
\end{array}\right.
$$

Definition 7.1. If $N$ is a positive integer (with $4 \mid N$ if $k \in \frac{1}{2} \mathbb{Z} \backslash \mathbb{Z}$ ), then a weight $k$ harmonic Maass form on $\Gamma \in\left\{\Gamma_{1}(N), \Gamma_{0}(N)\right\}$ is any smooth function $M: \mathbb{H} \rightarrow \mathbb{C}$ satisfying the following:

(1) For all $A=\left(\begin{array}{ll}a & b \\ c & d\end{array}\right) \in \Gamma$ and all $z \in \mathbb{H}$, we have

$$
M\left(\frac{a z+b}{c z+d}\right)= \begin{cases}(c z+d)^{k} M(z) & \text { if } k \in \mathbb{Z}, \\ \left(\frac{c}{d}\right)^{2 k} \epsilon_{d}^{-2 k}(c z+d)^{k} M(z) & \text { if } k \in \frac{1}{2} \mathbb{Z} \backslash \mathbb{Z} .\end{cases}
$$

Here $\left(\frac{c}{d}\right)$ denotes the extended Legendre symbol, and $\sqrt{z}$ is the principal branch of the holomorphic square root.

(2) We have that $\Delta_{k} M=0$.

(3) There is a polynomial $P_{M}=\sum_{n \leq 0} c^{+}(n) q^{n} \in \mathbb{C}\left[q^{-1}\right]$ such that

$$
M(z)-P_{M}(z)=O\left(e^{-\epsilon y}\right)
$$

as $y \rightarrow+\infty$ for some $\epsilon>0$. Analogous conditions are required at all cusps.

REMARK 9. Maass forms and classical modular forms are required to satisfy moderate growth conditions at cusps, and it is for this reason that harmonic Maass forms are often referred to as "harmonic weak Maass forms". The term "weak" refers to the relaxed condition Definition 7.1 (3) which gives rise to a rich theory. For convenience, we use the terminology "harmonic Maass form" instead of "harmonic weak Maass form".

REMARK 10 . We refer to the polynomial $P_{M}$ as the principal part of $M(z)$ at $\infty$. Obviously, if $P_{M}$ is non-constant, then $M(z)$ has exponential growth at $\infty$. Similar remarks apply at all cusps.

Remark 11. Bruinier and Funke [63] define two types of harmonic Maass forms based on varying the growth conditions at cusps. For a group $\Gamma$, they refer to these spaces as $H_{k}(\Gamma)$ and $H_{k}^{+}(\Gamma)$. Definition 7.1 (3) corresponds to their $H_{k}^{+}(\Gamma)$ definition.

REMARK 12. Since holomorphic functions on $\mathbb{H}$ are harmonic, it follows that weakly holomorphic modular forms are harmonic Maass forms. 
REMARK 13. Here we recall the congruence subgroups. If $N$ is a positive integer, then define the level $N$ congruence subgroups $\Gamma_{0}(N), \Gamma_{1}(N)$, and $\Gamma(N)$ by

$$
\begin{aligned}
\Gamma_{0}(N): & =\left\{\left(\begin{array}{ll}
a & b \\
c & d
\end{array}\right) \in \mathrm{SL}_{2}(\mathbb{Z}): c \equiv 0 \bmod N\right\}, \\
\Gamma_{1}(N): & =\left\{\left(\begin{array}{ll}
a & b \\
c & d
\end{array}\right) \in \mathrm{SL}_{2}(\mathbb{Z}): a \equiv d \equiv 1 \quad \bmod N, \text { and } c \equiv 0 \quad \bmod N\right\}, \\
\Gamma(N): & =\left\{\left(\begin{array}{ll}
a & b \\
c & d
\end{array}\right) \in \mathrm{SL}_{2}(\mathbb{Z}): a \equiv d \equiv 1 \bmod N, \text { and } b \equiv c \equiv 0 \bmod N\right\} .
\end{aligned}
$$

REMARK 14. For $k \in \frac{1}{2} \mathbb{Z} \backslash \mathbb{Z}$, the transformation law in Definition 7.1 (1) coincides with those in Shimura's theory of half-integral weight modular forms [192].

REMARK 15. Later we shall require the classical "slash" operator. For convenience, we recall its definition here. Suppose that $k \in \frac{1}{2} \mathbb{Z}$. For $A=$ $\left(\begin{array}{ll}a & b \\ c & d\end{array}\right) \in \mathrm{SL}_{2}(\mathbb{Z})\left(\Gamma_{0}(4)\right.$ if $\left.k \in \frac{1}{2} \mathbb{Z} \backslash \mathbb{Z}\right)$, define $j(A, z)$ by

$$
j(A, z):= \begin{cases}\sqrt{c z+d} & \text { if } k \in \mathbb{Z}, \\ \left(\frac{c}{d}\right) \varepsilon_{d}^{-1} \sqrt{c z+d} & \text { if } k \in \frac{1}{2} \mathbb{Z} \backslash \mathbb{Z},\end{cases}
$$

where $\varepsilon_{d}$ is defined by (7.2), and where $\sqrt{z}$ is the principal branch of the holomorphic square root as before. For functions $f: \mathbb{H} \rightarrow \mathbb{C}$, we define the action of the "slash" operator by

$$
\left(\left.f\right|_{k} A\right)(z):=j(A, z)^{-2 k} f(A z)=j(A, z)^{-2 k} f\left(\frac{a z+b}{c z+d}\right) .
$$

Notice that Definition 7.1 (1) may be rephrased as

$$
\left(\left.M\right|_{k} A\right)(z)=M(z) \text {. }
$$

REMARK 16. We shall also consider level $N$ weight $k \in \frac{1}{2} \mathbb{Z}$ forms with Nebentypus $\chi$. To define such forms, suppose that $N$ is a positive integer (with $4 \mid N$ if $k \in \frac{1}{2} \mathbb{Z} \backslash \mathbb{Z}$ ), and let $\chi$ be a Dirichlet character modulo $N$. To define these forms, one merely requires, ${ }^{14}$ for every $\left(\begin{array}{ll}a & b \\ c & d\end{array}\right) \in \Gamma_{0}(N)$, that

$$
M\left(\frac{a z+b}{c z+d}\right)= \begin{cases}\chi(d)(c z+d)^{k} M(z) & \text { if } k \in \mathbb{Z}, \\ \left(\frac{c}{d}\right)^{2 k} \epsilon_{d}^{-2 k} \chi(d)(c z+d)^{k} M(z) & \text { if } k \in \frac{1}{2} \mathbb{Z} \backslash \mathbb{Z} .\end{cases}
$$

Throughout, we shall adopt the following notation. If $\Gamma \subset \mathrm{SL}_{2}(\mathbb{Z})$ is a congruence subgroup, then we let

$$
\begin{aligned}
S_{k}(\Gamma) & :=\text { weight } k \text { cusp forms on } \Gamma \\
M_{k}(\Gamma) & :=\text { weight } k \text { holomorphic modular forms on } \Gamma, \\
M_{k}^{!}(\Gamma) & :=\text { weight } k \text { weakly holomorphic modular forms on } \Gamma, \\
H_{k}(\Gamma) & :=\text { weight } k \text { harmonic Maass forms on } \Gamma .
\end{aligned}
$$

\footnotetext{
${ }^{14}$ This replaces (1) in Definition 7.1.
} 
Furthermore, if $\chi$ is a Dirichlet character modulo $N$, then we let

$S_{k}(N, \chi):=$ level $N$ weight $k$ cusp forms with Nebentypus $\chi$,

$M_{k}(N, \chi):=$ level $N$ weight $k$ holomorphic modular forms with Nebentypus $\chi$,

$M_{k}^{!}(N, \chi):=$ level $N$ weight $k$ weakly holomorphic modular forms with Nebentypus $\chi$,

$H_{k}(N, \chi):=$ level $N$ weight $k$ harmonic Maass forms with Nebentypus $\chi$.

When the Nebentypus character is trivial, we shall suppress $\chi$ from the notation.

The real analytic forms in Theorem 6.1 provide non-trivial examples of weight $1 / 2$ harmonic Maass forms. More generally, the work of Zwegers $[\mathbf{2 1 8}, \mathbf{2 1 9}]$, shows how to complete all of Ramanujan's mock theta functions to obtain weight $1 / 2$ harmonic Maass forms. In Section 8, we shall present further examples of harmonic Maass forms.

7.2. Fourier expansions. In this paper we consider harmonic Maass forms with weight $2-k \in \frac{1}{2} \mathbb{Z}$ with $k>1$. Therefore, throughout we assume that $1<k \in \frac{1}{2} \mathbb{Z}$.

Harmonic Maass forms have distinguished Fourier expansions which are described in terms of the incomplete Gamma-function $\Gamma(\alpha ; x)$

$$
\Gamma(\alpha ; x):=\int_{x}^{\infty} e^{-t} t^{\alpha-1} d t
$$

and the usual parameter $q:=e^{2 \pi i z}$. The following characterization is straightforward (for example, see Section 3 of [63]).

LEMma 7.2. Assume the notation and hypotheses above, and suppose that $N$ is a positive integer. If $f(z) \in H_{2-k}\left(\Gamma_{1}(N)\right)$, then its Fourier expansion is of the form

$$
f(z)=\sum_{n \gg-\infty} c_{f}^{+}(n) q^{n}+\sum_{n<0} c_{f}^{-}(n) \Gamma(k-1,4 \pi|n| y) q^{n},
$$

where $z=x+i y \in \mathbb{H}$, with $x, y \in \mathbb{R}$.

As Lemma 7.2 reveals, $f(z)$ naturally decomposes into two summands. In view of this fact, we make the following definition.

Definition 7.3. Assuming the notation and hypotheses in Lemma 7.2, we refer to

$$
f^{+}(z):=\sum_{n \gg-\infty} c_{f}^{+}(n) q^{n}
$$

as the holomorphic part of $f(z)$, and we refer to

$$
f^{-}(z):=\sum_{n<0} c_{f}^{-}(n) \Gamma(k-1,4 \pi|n| y) q^{n}
$$

as the non-holomorphic part of $f(z)$. 
REMARK 17. A harmonic Maass form with trivial non-holomorphic part is a weakly holomorphic modular form. We shall make use of this fact as follows. If $f_{1}, f_{2} \in H_{2-k}(\Gamma)$ are two harmonic Maass forms with equal nonholomorphic parts, then $f_{1}-f_{2} \in M_{2-k}^{!}(\Gamma)$.

7.3. The $\xi$-operator and period integrals of cusp forms. Harmonic Maass forms are related to classical modular forms thanks to the properties of differential operators. The first nontrivial relationship depends on the differential operator

$$
\xi_{w}:=2 i y^{w} \cdot \overline{\frac{\partial}{\partial \bar{z}}}
$$

The following lemma, ${ }^{15}$ which is a straightforward refinement of a proposition of Bruinier and Funke (see Proposition 3.2 of [63]), shall play a central role throughout this paper.

Lemma 7.4. If $f \in H_{2-k}(N, \chi)$, then

$$
\xi_{2-k}: H_{2-k}(N, \chi) \longrightarrow S_{k}(N, \bar{\chi})
$$

is a surjective map. Moreover, assuming the notation in Definition 7.3, we have that

$$
\xi_{2-k}(f)=-(4 \pi)^{k-1} \sum_{n=1}^{\infty} \overline{c_{f}^{-}(-n)} n^{k-1} q^{n} .
$$

Thanks to Lemma 7.4, we are in a position to relate the non-holomorphic parts of harmonic Maass forms, the expansions

$$
f^{-}(z):=\sum_{n<0} c_{f}^{-}(n) \Gamma(k-1,4 \pi|n| y) q^{n},
$$

with "period integrals" of modular forms. This observation was critical in Zwegers's work on Ramanujan's mock theta functions.

To make this connection, we must relate the Fourier expansion of the cusp form $\xi_{2-k}(f)$ with $f^{-}(z)$. This connection is made by applying the simple integral identity

$$
\int_{-\bar{z}}^{i \infty} \frac{e^{2 \pi i n \tau}}{(-i(\tau+z))^{2-k}} d \tau=i(2 \pi n)^{1-k} \cdot \Gamma(k-1,4 \pi n y) q^{-n} .
$$

This identity follows by the direct calculation

$\int_{-\bar{z}}^{i \infty} \frac{e^{2 \pi i n \tau}}{(-i(\tau+z))^{2-k}} d \tau=\int_{2 i y}^{i \infty} \frac{e^{2 \pi i n(\tau-z)}}{(-i \tau)^{2-k}} d \tau=i(2 \pi n)^{1-k} \cdot \Gamma(k-1,4 \pi n y) q^{-n}$.

In this way, we may think of the non-holomorphic parts of weight $2-k$ harmonic Maass forms as period integrals of weight $k$ cusp forms, where one applies (7.8) to

$$
\int_{-\bar{z}}^{i \infty} \frac{\sum_{n=1}^{\infty} a(n) e^{2 \pi i n \tau}}{(-i(\tau+z))^{2-k}} d \tau
$$

\footnotetext{
${ }^{15}$ The formula for $\xi_{2-k}(f)$ corrects a typographical error in [63].
} 
where $\sum_{n=1}^{\infty} a(n) q^{n}$ is a weight $k$ cusp form. In short, $f^{-}(z)$ is the period integral of the cusp form $\xi_{2-k}(f)$.

In addition to this important observation, we require the following fact concerning the nontriviality of certain principal parts of harmonic Maass forms.

LEMma 7.5. If $f \in H_{2-k}(\Gamma)$ has the property that $\xi_{2-k}(f) \neq 0$, then the principal part of $f$ is nonconstant for at least one cusp.

Sketch of the Proof. This lemma follows from the work of Bruinier and Funke $[\mathbf{6 3}]$. Using their pairing $\{\bullet \bullet \bullet\}$, one finds that $\left\{\xi_{2-k} f, f\right\} \neq 0$ thanks to its interpretation in terms of Petersson norms. On the other hand, Proposition 3.5 of $[\mathbf{6 3}]$ expresses this quantity in terms of the principal part of $f$ and the coefficients of the cusp form $\xi_{2-k}(f)$. An inspection of this formula reveals that at least one principal part of $f$ must be nonconstant.

7.4. The $\boldsymbol{D}$-operator. In addition to the differential operator $\xi_{2-k}$, which defines the surjective map

$$
\xi_{2-k}: H_{2-k}(N, \chi) \longrightarrow S_{k}(N, \bar{\chi}),
$$

we consider the differential operator

$$
D:=\frac{1}{2 \pi i} \cdot \frac{d}{d z}
$$

We have the following theorem for integer weights.

Theorem 7.6. Suppose that $2 \leq k \in \mathbb{Z}$ and $f \in H_{2-k}(N)$, then

$$
D^{k-1}(f) \in M_{k}^{!}(N) \text {. }
$$

Moreover, assuming the notation in (7.6), we have

$$
D^{k-1} f=D^{k-1} f^{+}=\sum_{n \gg-\infty} c_{f}^{+}(n) n^{k-1} q^{n} .
$$

To prove this theorem, we must first recall some further differential operators, the Maass raising and lowering operators (for example, see $[\mathbf{6 3}, \mathbf{7 1}]$ ) $R_{k}$ and $L_{k}$. They are defined by

$$
\begin{aligned}
& R_{k}=2 i \frac{\partial}{\partial z}+k y^{-1}=i\left(\frac{\partial}{\partial x}-i \frac{\partial}{\partial y}\right)+k y^{-1}, \\
& L_{k}=-2 i y^{2} \frac{\partial}{\partial \bar{z}}=-i y^{2}\left(\frac{\partial}{\partial x}+i \frac{\partial}{\partial y}\right) .
\end{aligned}
$$

With respect to the Petersson slash operator (7.4), these operators satisfy the intertwining properties

$$
\begin{aligned}
R_{k}\left(\left.f\right|_{k} \gamma\right) & =\left.\left(R_{k} f\right)\right|_{k+2} \gamma, \\
L_{k}\left(\left.f\right|_{k} \gamma\right) & =\left.\left(L_{k} f\right)\right|_{k-2} \gamma,
\end{aligned}
$$


for any $\gamma \in \mathrm{SL}_{2}(\mathbb{R})$. The Laplacian $\Delta_{k}$ can be expressed in terms of $R_{k}$ and $L_{k}$ by

$$
-\Delta_{k}=L_{k+2} R_{k}+k=R_{k-2} L_{k} .
$$

If $f$ is an eigenfunction of $\Delta_{k}$ satisfying $\Delta_{k} f=\lambda f$, then

$$
\begin{aligned}
\Delta_{k+2} R_{k} f & =(\lambda+k) R_{k} f, \\
\Delta_{k-2} L_{k} f & =(\lambda-k+2) L_{k} f .
\end{aligned}
$$

For any positive integer $n$ we put

$$
R_{k}^{n}:=R_{k+2(n-1)} \circ \cdots \circ R_{k+2} \circ R_{k} .
$$

We also let $R_{k}^{0}$ be the identity. The differential operator $D:=\frac{1}{2 \pi i} \frac{d}{d z}=q \frac{d}{d q}$ satisfies the following relation

$$
R_{k}=-4 \pi D+k / y \text {. }
$$

The next well known lemma (for example, see (4.15) of [148]) is often referred to as Bol's identity.

Lemma 7.7. Assuming the notation and hypotheses above, we have

$$
D^{k-1}=\frac{1}{(-4 \pi)^{k-1}} R_{2-k}^{k-1}
$$

By Lemma 7.7, we see that $D^{k-1}$ defines a linear map

$$
D^{k-1}: M_{2-k}^{!}(N) \longrightarrow M_{k}^{!}(N) \text {. }
$$

Theorem 7.6 asserts that this map extends to harmonic Maass forms. Moreover, the theorem provides a simple description of the images.

Proof of TheOREM 7.6. It is clear that $D^{k-1} f$ has the transformation behavior of a modular form of weight $k$.

We now show that $L_{k} D^{k-1} f=0$. This implies that $D^{k-1} f$ is holomorphic on $\mathbb{H}$. By Lemma 7.7, it suffices to show that $L_{k} R_{2-k}^{k-1} f=0$. Since $\Delta_{2-k} f=0$, it follows from (7.11) by induction that

$$
\Delta_{k-2} R_{2-k}^{k-2} f=(2-k) R_{2-k}^{k-2} f .
$$

Using (7.10), we obtain

$$
L_{k} R_{2-k}^{k-1} f=\left(L_{k} R_{k-2}\right) R_{2-k}^{k-2} f=\left(-\Delta_{k-2}-(k-2)\right) R_{2-k}^{k-2} f=0 .
$$

Finally, the growth behavior of $f$ at the cusps implies that $D^{k-1} f$ is meromorphic at the cusps. Therefore, $D^{k-1}$ indeed extends to $H_{2-k}(N)$.

To complete the proof, we compute the Fourier expansion of $D^{k-1} f$. Assuming the notation in (7.6), a straightforward calculation gives

$$
\begin{aligned}
R_{2-k}^{k-2} f(z)= & \sum_{n \gg-\infty} c_{f}^{+}(n) \Gamma(k-1,4 \pi n y)(-y)^{2-k} e^{2 \pi i n \bar{z}} \\
& +(k-2) !^{2} \sum_{n<0} c_{f}^{-}(n)(-y)^{2-k} e^{2 \pi i n \bar{z}}
\end{aligned}
$$


Moreover, $R_{2-k}^{k-1} f$ has the Fourier expansion

$$
R_{2-k}^{k-1} f(z)=\sum_{n \gg-\infty} c_{f}^{+}(n)(-4 \pi n)^{k-1} q^{n} .
$$

In particular, we have

$$
D^{k-1} f=D^{k-1} f^{+}=\sum_{n \gg-\infty} c_{f}^{+}(n) n^{k-1} q^{n} .
$$

The first two formulas follow from the Fourier expansion of $f$ and the differential equations $\Delta_{k-2} R_{2-k}^{k-2} f=(2-k) R_{2-k}^{k-2} f$ and $\Delta_{k} R_{2-k}^{k-1} f=0$. The third formula is a consequence of the second and Lemma 7.7.

REMARK 18. Note that $g:=y^{k-2} \overline{R_{2-k}^{k-2} f}$ is a harmonic Maass form of weight $2-k$ in the (slightly more general) sense of Section 3 of [63]. Moreover, $\xi_{2-k} g=y^{-k} \overline{L_{2-k} g}=R_{2-k}^{k-1} f$. This can also be used to compute the Fourier expansions in the proof of Theorem 7.6.

Theorem 7.6 implies that the coefficients $c_{f}^{+}(n)$, for non-zero $n$, are obtained by dividing the $n$th coefficient of some fixed weakly holomorphic modular form by $n^{k-1}$. Therefore we are compelled to determine the image of the map

$$
D^{k-1}: H_{2-k}(N) \longrightarrow M_{k}^{!}(N)
$$

It is not difficult to see that this map is not generally surjective. Our next result determines the image of $D^{k-1}$ in terms of regularized inner products. The following result is obtained by Bruinier, Rhoades and the author in [68].

TheOrem 7.8. If $2 \leq k \in \mathbb{Z}$, then the image of the map

$$
D^{k-1}: H_{2-k}(N) \longrightarrow M_{k}^{!}(N)
$$

consists of those $h \in M_{k}^{!}(N)$ which are orthogonal to cusp forms with respect to the regularized inner product, which also have constant term 0 at all cusps of $\Gamma_{0}(N)$.

To make Theorem 7.8 precise, we must define what it means for a weakly holomorphic modular form to be orthogonal to cusp forms. To this end, we first recall the regularized inner product.

We stress again that $k \geq 2$ is an integer. Obviously, $\Gamma_{0}(N)$ has finite index in $\Gamma(1)=\mathrm{SL}_{2}(\mathbb{Z})$. We define a regularized inner product of $g \in M_{k}(N)$ and $h \in M_{k}^{!}(N)$ as follows. For $T>0$ we denote by $\mathcal{F}_{T}(\Gamma(1))$ the truncated fundamental domain

$$
\mathcal{F}_{T}(\Gamma(1))=\{z \in \mathbb{H}:|x| \leq 1 / 2,|z| \geq 1, \text { and } y \leq T\}
$$

for $\Gamma(1)$. Moreover, we define the truncated fundamental domain for $\Gamma_{0}(N)$ by

$$
\mathcal{F}_{T}(N)=\bigcup_{\gamma \in \Gamma_{0}(N) \backslash \Gamma(1)} \gamma \mathcal{F}_{T}(\Gamma(1))
$$


Following Borcherds [44], we define the regularized inner product $(g, h)^{r e g}$ as the constant term in the Laurent expansion at $s=0$ of the meromorphic continuation in $s$ of the function

$$
\frac{1}{\left[\Gamma(1): \Gamma_{0}(N)\right]} \lim _{T \rightarrow \infty} \int_{\mathcal{F}_{T}(N)} g(z) \overline{h(z)} y^{k-s} \frac{d x d y}{y^{2}} .
$$

Arguing as in Section 6 of [44], it can be shown that $(g, h)^{r e g}$ exists for any $g \in M_{k}(N)$ and $h \in M_{k}^{!}(N)$. (It also exists for $g \in M_{k}(N)$ and $h \in$ $H_{k}(N)$. But note that it does not exist in general if $g$ and $h$ are both weakly holomorphic with poles at the cusps.) For cusp forms $g$ and $h$, the regularized inner product reduces to the classical Petersson inner product.

REMARK 19. If $h \in M_{k}^{!}(N)$ has vanishing constant term at every cusp of $\Gamma_{0}(N)$, then

$$
(g, h)^{r e g}=\frac{1}{\left[\Gamma(1): \Gamma_{0}(N)\right]} \lim _{T \rightarrow \infty} \int_{\mathcal{F}_{T}(N)} g(z) \overline{h(z)} y^{k} \frac{d x d y}{y^{2}} .
$$

The following theorem provides a formula for the regularized inner product in terms of Fourier coefficients.

Theorem 7.9. If $g \in M_{k}(N)$ and $f \in H_{2-k}(N)$, then

$$
\left(g, R_{2-k}^{k-1} f\right)^{r e g}=\frac{(-1)^{k}}{\left[\Gamma(1): \Gamma_{0}(N)\right]} \sum_{\kappa \in \Gamma_{0}(N) \backslash P^{1}(\mathbb{Q})} w_{\kappa} \cdot c_{g}(0, \kappa) \overline{c_{f}^{+}(0, \kappa)},
$$

where $c_{g}(0, \kappa)$ (resp. $c_{f}^{+}(0, \kappa)$ ) denotes the constant term of the Fourier expansion of $g$ (resp. $f$ ) at the cusp $\kappa \in P^{1}(\mathbb{Q})$, and $w_{\kappa}$ is the width of the cusp $\kappa$.

Proof. For simplicity, we carry out the proof only in the special case $\Gamma_{0}(1)=\mathrm{SL}_{2}(\mathbb{Z})$. We put $H:=y^{k-2} \overline{R_{2-k}^{k-2} f}$, and let $h:=R_{2-k}^{k-1} f=y^{-k} \overline{L_{2-k} H}$. Since the constant terms at all cusps of $h$ vanish, we have

$$
\begin{aligned}
\left(g, R_{2-k}^{k-1} f\right)^{r e g} & =\lim _{T \rightarrow \infty} \int_{\mathcal{F}_{T}(1)} g(z) \overline{h(z)} y^{k} \frac{d x d y}{y^{2}} \\
& =\lim _{T \rightarrow \infty} \int_{\mathcal{F}_{T}(1)} g(z)\left(L_{2-k} H\right) \frac{d x d y}{y^{2}} \\
& =\lim _{T \rightarrow \infty} \int_{\mathcal{F}_{T}(1)} g(z)\left(\frac{\partial}{\partial \bar{z}} H\right) d z d \bar{z} \\
& =-\lim _{T \rightarrow \infty} \int_{\mathcal{F}_{T}(1)}(\bar{\partial} H) \wedge g(z) d z .
\end{aligned}
$$


Using the holomorphy of $g$, we obtain, by Stokes' theorem, the expression

$$
\begin{aligned}
\left(g, R_{2-k}^{k-1} f\right)^{r e g} & =-\lim _{T \rightarrow \infty} \int_{\mathcal{F}_{T}(1)} d(H(z) g(z) d z) \\
& =-\lim _{T \rightarrow \infty} \int_{\partial \mathcal{F}_{T}(1)} H(z) g(z) d z \\
& =\lim _{T \rightarrow \infty} \int_{x=-1 / 2}^{1 / 2} H(x+i T) g(x+i T) d x
\end{aligned}
$$

The integral over $x$ gives the constant term in the expansion of $H(x+$ $i T) g(x+i T)$, and it can be computed using the Fourier expansion

$$
\begin{aligned}
H(z)= & (-1)^{k} \sum_{n \gg-\infty} \overline{c_{f}^{+}(n)} \Gamma(k-1,4 \pi n y) e^{-2 \pi i n z} \\
& +(-1)^{k}(k-2) !^{2} \sum_{n<0} \overline{c_{f}^{-}(n)} e^{-2 \pi i n z}
\end{aligned}
$$

of $H$ (see the proof of Theorem 7.6) and the expansion of $g$. To complete the proof, notice that in the limit $T \rightarrow \infty$ the only contribution is $(-1)^{k} c_{g}(0) \overline{c_{f}^{+}(0)}$

Proof of Theorem 7.8. By Theorem 7.9, it follows that if $g \in S_{k}(N)$, then

$$
\left(g, R_{2-k}^{k-1} f\right)^{r e g}=0 .
$$

Therefore, if $f \in H_{2-k}(N)$, it follows from Lemma 7.7 and Theorem 7.9 that $D^{k-1} f$ is orthogonal to cusp forms.

Conversely, assume that $h \in M_{k}^{!}(N)$ is orthogonal to cusp forms and has vanishing constant term at every cusp of $\Gamma_{0}(N)$. By Lemma 3.11 of [63], we may chose $f \in H_{2-k}(N)$ such that the principal parts of $D^{k-1} f$ and $h$ at the cusps agree up to the constant terms. Since the constant terms of $h$ and $D^{k-1} f$ vanish, they trivially agree as well. Consequently,

$$
h-D^{k-1} f \in S_{k}(N) .
$$

In view of Theorem 7.9 and the hypothesis on $h$, we find that $h-D^{k-1} f$ is orthogonal to cusp forms, and so it is zero.

7.5. Hecke operators. It is natural to investigate the action of the Hecke operators on harmonic Maass forms. Here we illustrate how to combine facts about Hecke operators with Lemma 7.4 to obtain weakly holomorphic modular forms from harmonic Maass forms.

First we recall the classical Hecke operators. Suppose that $p$ is prime, and that $k \in \frac{1}{2} \mathbb{Z}$. Suppose that

$$
F(z)=\sum_{n \gg-\infty} a_{F}(n) q^{n}
$$


is a weight $k$ weakly holomorphic modular form on $\Gamma_{0}(N)$ with Nebentypus $\chi$. If $k \in \mathbb{Z}$, then the Hecke operator $T_{k}(p)$ is defined by

$$
F \mid T_{k}(p):=\sum_{n \gg-\infty}\left(a_{F}(p n)+\chi(p) p^{k-1} a_{F}(n / p)\right) q^{n} .
$$

If $k=\lambda+\frac{1}{2} \in \frac{1}{2} \mathbb{Z} \backslash \mathbb{Z}$, then we have that

$$
\begin{aligned}
F \mid T_{k}(p):= & \sum_{n \gg-\infty}\left(a_{F}\left(p^{2} n\right)+\chi(p)\left(\frac{(-1)^{\lambda} n}{p}\right) p^{\lambda-1} a_{F}(n)\right. \\
& \left.+\chi\left(p^{2}\right) p^{2 \lambda-1} a_{F}\left(n / p^{2}\right)\right) q^{n} .
\end{aligned}
$$

REMARK 20. For the sake of uniformity, we abuse notation in the case of the half-integral weight Hecke operators. Our operator $T_{k}(p)$ is traditionally referred to as $T\left(p^{2}\right)$ or $T_{k}\left(p^{2}\right)$.

These operators map modular forms to modular forms, and they define linear transformations on $M_{k}(N, \chi)$ and $S_{k}(N, \chi)$. Using Lemma 7.2, it is not difficult to see that the definitions of these Hecke operators extend to harmonic Maass forms and their spaces in the obvious way. Using Lemmas 7.2 and 7.4, it is then not difficult to obtain the following useful theorem. As before, here we suppose that $2-k \in \frac{1}{2} \mathbb{Z}$ with $k>1$.

TheOREM 7.10. Suppose that $f(z) \in H_{2-k}(N, \chi)$, and that $p \nmid N$ is a prime for which $\xi_{2-k}(f) \in S_{k}(N, \bar{\chi})$ is an eigenform of $T_{k}(p)$ with eigenvalue $\lambda(p)$.

(1) If $k \in \frac{1}{2} \mathbb{Z} \backslash \mathbb{Z}$, then

$$
f \mid T_{2-k}(p)-p^{2-2 k} \lambda(p) f
$$

is a weakly holomorphic modular form in $M_{2-k}^{!}(N, \chi)$.

(2) If $k \in \mathbb{Z}$, then

$$
f \mid T_{2-k}(p)-p^{1-k} \lambda(p) f
$$

is a weakly holomorphic modular form in $M_{2-k}^{!}(N, \chi)$.

\section{Examples of harmonic Maass forms}

In this section we present further examples of harmonic Maass forms. The interested reader should consult Zwegers's works $[\mathbf{2 1 8}, \mathbf{2 1 9}]$ to see those weight 1/2 harmonic Maass forms whose holomorphic parts are given in terms of Lambert-type series and generating functions for indefinite binary quadratic forms. Recent work of Bringmann, Folsom, and the author [49] concerns further properties of these forms, and it also gives related weight $3 / 2$ harmonic Maass forms. All of these harmonic Maass forms $f$ have the property that $\xi_{w}(f)$ is a linear combination of single variable theta functions.

Here we consider further examples of harmonic Maass forms. We recall the classical Eisenstein series $E_{2}(z)$, Zagier's weight $3 / 2$ Eisenstein series, 
certain Maass-Poincaré series, and a combinatorial power series which plays a central role in the Dyson-Ramanujan theory of partition congruences.

8.1. The Eisenstein series $\boldsymbol{E}_{\mathbf{2}}(\boldsymbol{z})$. The differential operator $D:=$ $\frac{1}{2 \pi i} \frac{d}{d z}=q \cdot \frac{d}{d q}$ plays an important role in the theory of modular forms and $q$-series. Indeed, as an operator on formal power series we have that

$$
D\left(\sum_{n=h}^{\infty} a(n) q^{n}\right):=\sum_{n=h}^{\infty} n a(n) q^{n} .
$$

Ramanujan proved $[\mathbf{1 7 2}]$ that

$$
D\left(E_{4}\right)=\left(E_{4} E_{2}-E_{6}\right) / 3 \quad \text { and } \quad D\left(E_{6}\right)=\left(E_{6} E_{2}-E_{8}\right) / 2,
$$

where $E_{k}$, for every even integer $k \geq 2$, is the standard Eisenstein series

$$
E_{k}(z):=1-\frac{2 k}{B_{k}} \sum_{n=1}^{\infty} \sigma_{k-1}(n) q^{n} .
$$

Here $B_{k}$ denotes the usual $k$ th Bernoulli number and $\sigma_{k-1}(n):=\sum_{d \mid n} d^{k-1}$.

As is well known, for even $k \geq 4$ we have that $E_{k}(z)$ is a weight $k$ modular form on $\mathrm{SL}_{2}(\mathbb{Z})$. However, the Eisenstein series $E_{2}(z)$

$$
E_{2}(z)=1-24 \sum_{n=1}^{\infty} \sigma_{1}(n) q^{n}
$$

is not modular, and so the derivative of a modular form is not (in general) a modular form.

It is not difficult to pin down the obstruction which prevents $E_{2}(z)$ from being a modular form. Indeed, it is simple to show (for example, see page 113 of $[\mathbf{1 3 4}])$, for $z \in \mathbb{H}$, that

$$
z^{-2} E_{2}(-1 / z)=E_{2}(z)+\frac{6}{\pi i z}
$$

Using this fact, it is not difficult to modify $E_{2}(z)$ to obtain a weight 2 harmonic Maass form. Using (8.1), and the fact that

$$
\mathrm{SL}_{2}(\mathbb{Z})=\left\langle\left(\begin{array}{ll}
1 & 1 \\
0 & 1
\end{array}\right),\left(\begin{array}{cc}
0 & 1 \\
-1 & 0
\end{array}\right)\right\rangle
$$

one finds that

$$
E_{2}^{*}(z):=-\frac{3}{\pi y}+E_{2}(z)
$$

where $y=\operatorname{Im}(z)$, is a weight 2 harmonic Maass form on $\mathrm{SL}_{2}(\mathbb{Z})$ in the more general sense of Bruinier and Funke. It fails to satisfy Definition 7.1 (3). Obviously, its holomorphic part is $E_{2}(z)$, and its non-holomorphic part is the simple function $-\frac{3}{\pi y}$. 
REMARK 21. Although $E_{2}^{*}(z)$ is not a harmonic Maass form in the strict sense of Definition 7.1, it still behaves well under the differential operator $\xi_{2}$. One easily checks that $\xi_{2}\left(E_{2}^{*}(z)\right)$ is constant, and so it is a modular form of weight 0 . This is in line with Lemma 7.4.

8.2. Zagier's Eisenstein series. In their work on the intersection theory of Hilbert modular surfaces $[\mathbf{1 2 3}, \mathbf{2 1 0}]$, Hirzebruch and Zagier required a non-holomorphic weight $3 / 2$ Eisenstein series $G(z)$ on $\Gamma_{0}(4)$. The nonholomorphic part of this Eisenstein series is essentially the "period integral" of the classical Jacobi theta function

$$
\Theta_{0}(z):=1+2 q+2 q^{4}+2 q^{9}+2 q^{16}+\cdots .
$$

This is analogous with the work of Zwegers on Ramanujan's mock theta functions (see Theorem 6.1 and the discussion after Lemma 7.4) in that the non-holomorphic parts of his harmonic Maass forms are also period integrals of single variable theta functions. Unlike Zwegers's harmonic Maass forms, where the holomorphic parts turned out to be combinatorial $q$-series such as the mock theta functions of Ramanujan, the holomorphic part of the Zagier form is the generating function for Hurwitz class numbers. More precisely, we have (in Zagier's notation) the following theorem.

THEOREM 8.1. If $H(0)=\zeta(-1)=-\frac{1}{12}$, and if $H(n)$ denotes the usual Hurwitz class numbers for discriminants $-n$, then the function

$$
G(z)=\sum_{n=0}^{\infty} H(n) q^{n}+\frac{1}{16 \pi \sqrt{y}} \sum_{n=-\infty}^{\infty} \beta\left(4 \pi n^{2} y\right) q^{-n^{2}}
$$

is a weight 3/2 harmonic Maass form on $\Gamma_{0}(4)$ in the sense of Bruinier and Funke. Here we have that $\beta(s):=\int_{1}^{\infty} t^{-3 / 2} e^{-s t} d t$.

REMARK 22. Zagier's $\beta$-function is essentially the incomplete Gammafunction.

As in the case of $E_{2}^{*}(z)$, we have that $G(z)$ is a weight $3 / 2$ harmonic Maass form on $\Gamma_{0}(4)$ in the more general sense of Bruinier and Funke. Again, the culprit is Definition $7.1(3)$. Nevertheless, one sees that $\xi_{\frac{3}{2}}(G)$ is a multiple of $\Theta_{0}(z)$, which is in line with the phenomenon in Lemma 7.4. The only difference is that $\Theta_{0}(z)$ is not a cusp form.

REMARK 23. In recent work, Bringmann and Lovejoy [51] have found a striking identity relating a certain rank generating function for overpartitions to $G(z)$.

8.3. Poincaré series. Here we describe certain Maass-Poincaré series. Throughout, we rely on classical special functions whose properties and definitions may be found in $[\mathbf{1}, \mathbf{2 0}]$. Such Poincaré series, which were first given by D. Hejhal, are discussed in $[56,62,65,117]$.

Suppose that $k \in \frac{1}{2} \mathbb{Z}$, and that $N$ is a positive integer (with $4 \mid N$ if $k \in \frac{1}{2} \mathbb{Z} \backslash \mathbb{Z}$ ). Let $m$ be an integer, and let $\varphi_{m}: \mathbb{R}^{+} \rightarrow \mathbb{C}$ be a function which 
satisfies $\varphi_{m}(y)=O\left(y^{\alpha}\right)$, as $y \rightarrow 0$, for some $\alpha \in \mathbb{R}$. If $e(\alpha):=e^{2 \pi i \alpha}$ as usual, then let

$$
\varphi_{m}^{*}(z):=\varphi_{m}(y) e(m x) .
$$

This function is fixed by the group of translations

$$
\Gamma_{\infty}:=\left\{ \pm\left(\begin{array}{ll}
1 & n \\
0 & 1
\end{array}\right): n \in \mathbb{Z}\right\}
$$

Using the slash operator (7.4), we define the Poincaré series $\mathbb{P}(m, k, N$, $\left.\varphi_{m} ; z\right)$ by

$$
\mathbb{P}\left(m, k, N, \varphi_{m} ; z\right):=\sum_{A \in \Gamma_{\infty} \backslash \Gamma_{0}(N)}\left(\left.\varphi_{m}^{*}\right|_{k} A\right)(z) .
$$

The Fourier expansions of these series are given in terms of the Kloosterman sums

$$
K_{k}(m, n, c):= \begin{cases}\sum_{v(c) \times} e\left(\frac{m \bar{v}+n v}{c}\right) & \text { if } k \in \mathbb{Z}, \\ \sum_{v(c) \times}\left(\frac{c}{v}\right)^{2 k} \varepsilon_{v}^{2 k} e\left(\frac{m \bar{v}+n v}{c}\right) & \text { if } k \in \frac{1}{2} \mathbb{Z} \backslash \mathbb{Z} .\end{cases}
$$

In the sums above, $v$ runs through the primitive residue classes modulo $c$, and $\bar{v}$ denotes the multiplicative inverse of $v$ modulo $c$. The following lemma gives the fundamental properties of such Poincaré series (for example, see Proposition 3.1 of [65] where $N=4$ ).

LEMma 8.2. If $k>2-2 \alpha$, then the following are true.

(1) Each Poincaré series $\mathbb{P}\left(m, k, N, \varphi_{m} ; z\right)$ is a weight $k \Gamma_{0}(N)$ invariant function.

(2) Near the cusp at $\infty$, the function $\mathbb{P}\left(m, k, N, \varphi_{m} ; z\right)-\varphi_{m}^{*}(z)$ has moderate growth. Near the other cusps, $\mathbb{P}\left(m, k, N, \varphi_{m} ; z\right)$ has moderate growth.

(3) If $\mathbb{P}\left(m, k, N, \varphi_{m} ; z\right)$ is twice continuously differentiable, then it has the locally uniformly absolutely convergent Fourier expansion

$$
\mathbb{P}\left(m, k, N, \varphi_{m} ; z\right)=\varphi_{m}^{*}(z)+\sum_{n \in \mathbb{Z}} a(n, y) e(n x),
$$

where

$$
\begin{aligned}
a(n, y):= & \sum_{\substack{c>0 \\
c \equiv 0}}^{\infty} c^{-k} K_{k}(m, n, c) \\
& \int_{-\infty}^{\infty} z^{-k} \varphi_{m}\left(\frac{y}{c^{2}|z|^{2}}\right) e\left(-\frac{m x}{c^{2}|z|^{2}}-n x\right) d x
\end{aligned}
$$

We now recall the classical family of Poincaré series (for example, see $[\mathbf{1 2 5}, \mathbf{1 6 7}]$ ) which arises from Lemma 8.2. If $2 \leq k \in \frac{1}{2} \mathbb{Z}, N$ is positive, and if $m$ is an integer, then let

$$
P(m, k, N ; z)=q^{m}+\sum_{n=1}^{\infty} a(m, k, N ; n) q^{n}=: \mathbb{P}(m, k, N, e(i m y) ; z) .
$$


These series are modular, and their Fourier expansions are given in terms of the $I$-Bessel and $J$-Bessel functions, and the Kloosterman sums above.

Theorem 8.3. If $2 \leq k \in \frac{1}{2} \mathbb{Z}$, and $m, N \geq 1$ (with $4 \mid N$ if $k \in \frac{1}{2} Z \backslash \mathbb{Z}$ ), then the following are true.

1) We have that $P(m, k, N ; z) \in S_{k}(N)$, and for positive integers $n$ we have

$$
\begin{aligned}
a(m, k, N ; n)= & 2 \pi(-1)^{\frac{k}{2}}\left(\frac{n}{m}\right)^{\frac{k-1}{2}} \cdot \sum_{\substack{c>0 \\
c \equiv 0}} \frac{K_{k}(m, n, c)}{c} \\
& \cdot J_{k-1}\left(\frac{4 \pi \sqrt{m n}}{c}\right) .
\end{aligned}
$$

2) We have that $P(-m, k, N ; z) \in M_{k}^{!}(N)$, and for positive integers $n$ we have

$$
\begin{aligned}
a(-m, k, N ; n)= & 2 \pi(-1)^{\frac{k}{2}}\left(\frac{n}{m}\right)^{\frac{k-1}{2}} \cdot \sum_{\substack{c>0 \\
(\bmod N)}} \frac{K_{k}(-m, n, c)}{c} \\
& \cdot I_{k-1}\left(\frac{4 \pi \sqrt{|m n|}}{c}\right) .
\end{aligned}
$$

Now we recall one family of Maass-Poincaré series which have appeared in earlier works $[\mathbf{6 2}, \mathbf{6 5}, \mathbf{1 1 7}, \mathbf{1 6 0}]$. To define them, again suppose that $2<k \in \frac{1}{2} \mathbb{Z}$, and that $N$ is a positive integer (with $4 \mid N$ if $k \in \frac{1}{2} \mathbb{Z} \backslash \mathbb{Z}$ ). To employ Lemma 8.2, we first select an appropriate function $\varphi$.

Let $M_{\nu, \mu}(z)$ be the usual $M$-Whittaker function which is a solution to the differential equation

$$
\frac{\partial^{2} u}{\partial z^{2}}+\left(-\frac{1}{4}+\frac{\nu}{z}+\frac{\frac{1}{4}-\mu^{2}}{z^{2}}\right) u=0 .
$$

For complex $s$, let

$$
\mathcal{M}_{s}(y):=|y|^{-\frac{k}{2}} M_{\frac{k}{2} \operatorname{sgn}(y), s-\frac{1}{2}}(|y|),
$$

and for positive $m$ let $\varphi_{-m}(z):=\mathcal{M}_{1-\frac{k}{2}}(-4 \pi m y)$. We now let

$$
Q(-m, k, N ; z):=\mathbb{P}\left(-m, 2-k, N, \varphi_{-m} ; z\right) .
$$

Lemma 8.2 leads to the following theorem (see $[\mathbf{5 4}, \mathbf{5 6}, \mathbf{6 2}, \mathbf{1 1 7}]$ ).

TheOrem 8.4. If $2<k \in \frac{1}{2} \mathbb{Z}$ and $m, N \geq 1$ (with $4 \mid N$ if $k \in \frac{1}{2} \mathbb{Z} \backslash \mathbb{Z}$ ), then $Q(-m, k, N ; z)$ is in $H_{2-k}(N)$. Moreover, if $I_{k-1}$ is the usual I-Bessel function, then

$Q(-m, k, N ; z)=(1-k)(\Gamma(k-1,4 \pi m y)-\Gamma(k-1)) q^{-m}+\sum_{n \in \mathbb{Z}} c_{m}(n, y) q^{n}$. 
1) If $n<0$, then

$$
\begin{aligned}
c_{m}(n, y)= & 2 \pi i^{k}(1-k) \Gamma(k-1,4 \pi|n| y)\left|\frac{n}{m}\right|^{\frac{1-k}{2}} \\
& \times \sum_{c \equiv 0} \frac{K_{2-k}(-m, n, c)}{c} \cdot J_{k-1}\left(\frac{4 \pi \sqrt{|m n|}}{c}\right) .
\end{aligned}
$$

2) If $n>0$, then

$$
\begin{aligned}
c_{m}(n, y)= & -2 \pi i^{k} \Gamma(k)\left(\frac{n}{m}\right)^{\frac{1-k}{2}} \\
& \times \sum_{c \equiv 0} \frac{K_{2-k}(-m, n, c)}{c} \cdot I_{k-1}\left(\frac{4 \pi \sqrt{|m n|}}{c}\right) .
\end{aligned}
$$

3) If $n=0$, then

$$
c_{m}(0, y)=-2^{k} \pi^{k} i^{k} m^{k-1} \sum_{\substack{c>0 \\ c \equiv 0}} \frac{K_{2-k}(-m, 0, c)}{c^{k}} .
$$

These three families of Poincaré series are closely related. The following theorem gives the explicit relationships between the cusp forms $P(m, k$, $N ; z)$, the weakly holomorphic modular forms $P(-m, k, N ; z)$, and the harmonic Maass forms $Q(-m, k, N ; z)$. They are related by the $\xi_{2-k}$ and $D^{k-1}$ differential operators.

TheOrem 8.5. Suppose that $2<k \in \frac{1}{2} \mathbb{Z}$, and that $m, N \geq 1$ (with $4 \mid N$ if $k \in \frac{1}{2} \mathbb{Z} \backslash \mathbb{Z}$ ). Then the following are true.

(1) Under the operator $\xi_{2-k}$, we have that

$$
\xi_{2-k}(Q(-m, k, N ; z))=(4 \pi)^{k-1} m^{k-1}(k-1) \cdot P(m, k, N ; z) \in S_{k}(N) .
$$

(2) Under the operator $D^{k-1}$, if $k$ is an integer, then we have that

$$
D^{k-1} Q(-m, k, N ; z)=-m^{k-1} \Gamma(k) \cdot P(-m, k, N ; z) \in M_{k}^{!}(N) .
$$

Proof. The claims follow easily from the explicit expansions in Theorems 8.3 and 8.4.

8.4. A family of weight $1 / 2$ harmonic Maass forms. In his work $[\mathbf{2 1 8}, \mathbf{2 1 9}]$ (also see Section 6.2), Zwegers describes the holomorphic parts of certain harmonic Maass forms in an explicit way. These series are presented as reciprocals of theta functions multiplied with Lambert-type series, and are also given as generating functions for indefinite theta series.

Here we present an infinite family of Maass forms which arise from the Eulerian series

$$
R(w ; q):=1+\sum_{n=1}^{\infty} \frac{q^{n^{2}}}{(w q ; q)_{n}\left(w^{-1} q ; q\right)_{n}},
$$


where, as before, we let

$$
(a ; q)_{n}:=(1-a)(1-a q) \cdots\left(1-a q^{n-1}\right) .
$$

To place this formal power series in context, we recall that we have already seen two important specializations in $w$. Thanks to Theorem 2.2, by setting $w=1$ we have that

$$
R(1 ; q)=\sum_{n=0}^{\infty} p(n) q^{n}
$$

By letting $w=-1$, we obtain the series

$$
R(-1 ; q)=1+\sum_{n=1}^{\infty} \frac{q^{n^{2}}}{(1+q)^{2}\left(1+q^{2}\right)^{2} \cdots\left(1+q^{n}\right)^{2}} .
$$

This series is Ramanujan's third order mock theta function $f(q)$, which by Theorem 6.1 is essentially the holomorphic part of a weight $1 / 2$ harmonic Maass form. In [55], Bringmann and the author generalized this fact.

To make this precise, suppose that $0<a<c$ are integers, and let $\zeta_{c}:=e^{2 \pi i / c}$. If $f_{c}:=\frac{2 c}{\operatorname{gcd}(c, 6)}$, then define the theta function $\Theta\left(\frac{a}{c} ; \tau\right)$ by

$$
\Theta\left(\frac{a}{c} ; \tau\right):=\sum_{m}(-1)^{m} \sin \left(\frac{a \pi(6 m+1)}{c}\right) \cdot \theta\left(6 m+1,6 f_{c} ; \frac{\tau}{24}\right),
$$

where

$$
\theta(\alpha, \beta ; \tau):=\sum_{n \equiv \alpha} n e^{2 \pi i \tau n^{2}} .
$$

Throughout, let $\ell_{c}:=\operatorname{lcm}\left(2 c^{2}, 24\right)$, and let $\widetilde{\ell}_{c}:=\ell_{c} / 24$. It is well known that $\Theta\left(\frac{a}{c} ; \ell_{c} \tau\right)$ is a cusp form of weight $3 / 2$ (for example, see [192]).

Using this cuspidal theta function, we define the function $S_{1}\left(\frac{a}{c} ; z\right)$ by the period integral

$$
S_{1}\left(\frac{a}{c} ; z\right):=\frac{-i \sin \left(\frac{\pi a}{c}\right) \ell_{c}^{\frac{1}{2}}}{\sqrt{3}} \int_{-\bar{z}}^{i \infty} \frac{\Theta\left(\frac{a}{c} ; \ell_{c} \tau\right)}{\sqrt{-i(\tau+z)}} d \tau .
$$

Using this notation, define $D\left(\frac{a}{c} ; z\right)$ by

$$
D\left(\frac{a}{c} ; z\right):=-S_{1}\left(\frac{a}{c} ; z\right)+q^{-\frac{\ell_{c}}{24}} R\left(\zeta_{c}^{a} ; q^{\ell_{c}}\right) .
$$

TheOREM 8.6. If $0<a<c$, where $c$ is odd, then $D\left(\frac{a}{c} ; z\right)$ is a Maass form of weight $1 / 2$ on $\Gamma_{1}\left(144 f_{c}^{2} \widetilde{\ell}_{c}\right)$.

REMARK 24. For even $c$, these Maass forms also satisfy transformation laws with respect to $\Gamma_{1}$ congruence subgroups. Our proof of Theorem 8.6 in [55] is long and complicated. It is based on a modification of earlier work of Watson $[\mathbf{2 0 6}, \mathbf{2 0 7}]$. Zagier $[\mathbf{2 1 3}]$ found a shorter proof of this result 
using a clever application of Zwegers's work on the $\widehat{\mu}$-function. His proof of Theorem 8.6 relies on the fact that

$$
\begin{aligned}
\frac{q^{-1 / 24} R(e(\alpha) ; q)}{e^{-\pi i \alpha}-e^{\pi i \alpha}}= & \frac{\eta(3 z)^{3} / \eta(z)}{\vartheta(3 \alpha ; 3 z)}-q^{-\frac{1}{6}} e(-\alpha) \mu(3 \alpha,-z ; 3 z) \\
& +q^{-\frac{1}{6}} e(\alpha) \mu(3 \alpha, z ; 3 z)
\end{aligned}
$$

\section{Dyson-Ramanujan theory of partition congruences}

As mentioned in the introduction, the partition function has been a "testing ground" for the theory of modular forms. In number theory we are interested in the divisibility properties of $p(n)$ and the size of $p(n)$. Here we consider the congruence properties of the partition function $p(n)$. It will turn out that these harmonic Maass forms have arithmetic properties which may be studied using the theory of $\ell$-adic Galois representations as developed by Deligne, Serre, and Swinnerton-Dyer [83, 187, 197]. These new congruences imply new partition congruences.

9.1. Ramanujan's congruences. Ramanujan proved the striking congruences

$$
\begin{aligned}
& p(5 n+4) \equiv 0 \quad(\bmod 5) \\
& p(7 n+5) \equiv 0 \quad(\bmod 7) \\
& p(11 n+6) \equiv 0 \quad(\bmod 11)
\end{aligned}
$$

and conjectured generalizations where the moduli are powers of 5, 7 and 11. These conjectures have been resolved in works by Atkin and Watson $[28,208]$.

These congruences are just the tip of the iceberg. In the late 1960s, Atkin [29] proved further congruences for small prime moduli. More recently, the author proved [162] that there are infinitely many such congruences for any prime modulus $Q \geq 5$. This result was subsequently generalized by $\mathrm{S}$. Ahlgren and the author $[\mathbf{2}, \mathbf{3}]$, and these results imply the following theorem.

THEOREM 9.1. If $M$ is coprime to 6 , then there are infinitely many non-nested arithmetic progressions $A n+B$ for which

$$
p(A n+B) \equiv 0 \quad(\bmod M) .
$$

REMARK 25. By non-nested, we mean that there are infinitely many arithmetic progressions $A n+B$, with $0 \leq B<A$, with the property that there are no progressions which contain another progression.

REMARK 26. It is believed that there are no such congruences with moduli which are not coprime to 6 (for example, see $[\mathbf{5}, \mathbf{1 6 3}]$ ). 
ExAmple 9.2. Here are the simplest examples of these congruences for the prime moduli $17 \leq M \leq 31$ :

$$
\begin{aligned}
& p(48037937 n+1122838) \equiv 0 \quad(\bmod 17), \\
& p(1977147619 n+815655) \equiv 0 \quad(\bmod 19), \\
& p(14375 n+3474) \equiv 0 \quad(\bmod 23), \\
& p(348104768909 n+43819835) \equiv 0 \quad(\bmod 29), \\
& p(4063467631 n+30064597) \equiv 0 \quad(\bmod 31) .
\end{aligned}
$$

9.2. Dyson's rank. In an effort to provide a combinatorial explanation of Ramanujan's congruences modulo 5 and 7, Dyson introduced [90] the socalled "rank" of a partition. The rank of a partition is defined to be its largest part minus the number of its parts. Let $N(m, n)$ denote the number of partitions of $n$ with rank $m$, and for integers $0 \leq r<t$ let $N(r, t ; n)$ denote the number of partitions of $n$ with rank congruent to $r$ modulo $t$. Dyson noticed that ranks modulo 5 (resp. 7) apppeared to divide the partitions of $5 n+4$ (resp. $7 n+5)$ into 5 (resp. 7 ) groups of equal size, thereby providing an elegant explanation for these two congruences of Ramanujan.

Atkin and Swinnerton-Dyer [31] confirmed Dyson's conjecture by proving the following result.

THEOREM 9.3. The following are true:

(1) If $0 \leq r<5$, then

$$
\sum_{n=0}^{\infty}\left(N(r, 5 ; 5 n+4)-\frac{p(5 n+4)}{5}\right) q^{n}=0 .
$$

(2) If $0 \leq r<7$, then

$$
\sum_{n=0}^{\infty}\left(N(r, 7 ; 7 n+5)-\frac{p(7 n+5)}{7}\right) q^{n}=0 .
$$

REMARK 27. Dyson [90] also noted that the rank fails to explain the Ramanujan congruence with modulus 11. A short calculation reveals that the first failure already occurs with $p(6)=11$. In view of this difficulty, Dyson further postulated the existence of another statistic, the so-called "crank", that could be used to provide an explanation for all three Ramanujan congruences. In 1988, Andrews and Garvan [25] found the crank, and they confirmed Dyson's speculation that it explains the three Ramanujan congruences. Recent work of Mahlburg [151] establishes that the AndrewsDyson-Garvan crank plays an even more central role in the theory partition congruences. His work establishes congruences modulo arbitrary powers of all primes $\geq 5$. Other work by Garvan, Kim and Stanton [99] gives a different "crank" for several other Ramanujan congruences. 
9.3. Maass form generating functions for Dyson's ranks. Although the rank does not explain Ramanujan's partition congruence with modulus 11, it turns out that it indeed plays a central role thanks to the arithmetic properties of certain harmonic Maass forms. Indeed, it turns out that the generating functions for $N(r, t ; n)$ are related to harmonic Maass forms. To make this connection, one simply generalizes the proof of Theorem 2.2 to obtain the following proposition concerning the power series

$$
R(w ; q):=1+\sum_{n=1}^{\infty} \frac{q^{n^{2}}}{(w q ; q)_{n}\left(w^{-1} q ; q\right)_{n}}
$$

considered earlier in (8.8).

Proposition 9.4. As a formal power series, we have that

$$
R(w ; q):=1+\sum_{n=1}^{\infty} \sum_{m=-\infty}^{\infty} N(m, n) w^{m} q^{n} .
$$

Thanks to Theorem 8.6, it is not difficult to relate the functions $N(r, t ; n)$ to the coefficients of weight $1 / 2$ harmonic Maass forms. In this direction, Bringmann and the author proved the following theorem in [55], which also sheds further light on Theorem 9.3.

TheOREm 9.5. Assume the notation from Section 8.4. If $0 \leq r<t$ are integers, where $t$ is odd, then

$$
\sum_{n=0}^{\infty}\left(N(r, t ; n)-\frac{p(n)}{t}\right) q^{\ell_{t} n-\tilde{\ell_{t}}}
$$

is the holomorphic part of a weight 1/2 harmonic Maass form on $\Gamma_{1}\left(144 f_{t}^{2} \widetilde{\ell}_{t}\right)$. Moreover, the non-holomorphic part of this Maass form is

$$
\frac{i \ell_{t}^{\frac{1}{2}}}{t \sqrt{3}} \sum_{m} \sum_{\left(\bmod f_{t}\right)} \sum_{n \equiv 6 m+1} A(r, t, m) \cdot \gamma(t, y ; n) q^{-\widetilde{\ell}_{t} n^{2}} .
$$

Here we have that

$$
A(r, t, m):=(-1)^{m} \sum_{j=1}^{t-1} \zeta_{t}^{-r j} \sin \left(\frac{\pi j}{t}\right) \sin \left(\frac{\pi j(6 m+1)}{t}\right),
$$

and we have that

$$
\gamma(t, y ; n):=\frac{i}{\sqrt{2 \pi \widetilde{\ell}_{t}}} \cdot \Gamma\left(\frac{1}{2} ; 4 \pi \widetilde{\ell}_{t} n^{2} y\right) .
$$

PROOF. This result follows easily from a standard argument involving the orthogonality of roots of unity. In particular, observe that

$$
\sum_{n=0}^{\infty} N(r, t ; n) q^{n}=\frac{1}{t} \sum_{n=0}^{\infty} p(n) q^{n}+\frac{1}{t} \sum_{j=1}^{t-1} \zeta_{t}^{-r j} \cdot R\left(\zeta_{t}^{j} ; q\right) .
$$


By Theorem 8.6, it then follows that

$$
\sum_{n=0}^{\infty}\left(N(r, t ; n)-\frac{p(n)}{t}\right) q^{\ell_{t} n-\frac{\ell_{t}}{24}}
$$

is the holomorphic part of a Maass form of weight $1 / 2$, one which is given as an appropriate weighted sum of the Maass forms $D\left(\frac{a}{t} ; z\right)$.

The result then follows from the explicit description of the non-holomorphic parts of these forms. For integers $0<a<t$, we have

$$
\begin{aligned}
& D\left(\frac{a}{t} ; z\right)=q^{-\frac{\ell_{t}}{24}}+\sum_{n=1}^{\infty} \sum_{m=-\infty}^{\infty} N(m, n) \zeta_{t}^{a m} q^{\ell_{t} n-\frac{\ell_{t}}{24}}+\frac{i \sin \left(\frac{\pi a}{t}\right) \ell_{t}^{\frac{1}{2}}}{\sqrt{3}} \\
& \times \sum_{m}(-1)^{m} \sin \left(\frac{a \pi(6 m+1)}{t}\right) \sum_{n \equiv 6 m+1}\left(\bmod 6 f_{t}\right) \\
& \gamma(t, y ; n) q^{-\widetilde{\ell_{t}} n^{2}}
\end{aligned}
$$

This expansion follows easily from

$$
\begin{aligned}
-S_{1}\left(\frac{a}{t} ; z\right)= & \frac{i \sin \left(\frac{\pi a}{t}\right) \ell_{t}^{\frac{1}{2}}}{\sqrt{3}} \sum_{m}(-1)^{m} \sin \left(\frac{a \pi(6 m+1)}{t}\right) \\
& \times \sum_{n \equiv 6 m+1}\left(\bmod 6 f_{t}\right) \\
\int_{-\bar{z}} & \frac{n e^{2 \pi i n^{2} \widetilde{\ell_{t}} \tau}}{\sqrt{-i(\tau+z)}} d \tau,
\end{aligned}
$$

and the identity (recall (7.8))

$$
\int_{-\bar{z}}^{i \infty} \frac{n e^{2 \pi i n^{2} \widetilde{\ell_{t}} \tau}}{\sqrt{-i(\tau+z)}} d \tau=\gamma(t, y ; n) \cdot q^{-\widetilde{\ell_{t}} n^{2}}
$$

REMARK 28. It is not difficult to deduce Theorem 9.3 from Theorem 9.5 using Atkin's $U$-operator. In short, one must merely prove that certain harmonic Maass forms, which turn out to be weakly holomorphic modular forms, are identically 0 .

Using quadratic twists, one can use Theorem 9.5 to obtain weakly holomorphic modular forms. Theorem 1.4 of [55] asserts the following result.

TheOREM 9.6. If $0 \leq r<t$ are integers, where $t$ is odd, and $\mathcal{P} \nmid 6 t$ is prime, then

$$
\sum_{\substack{n \geq 1 \\\left(\frac{24 \ell_{t} n-\ell_{t}}{\mathcal{P}}\right)=-\left(\frac{-24 \widetilde{\ell_{t}}}{\mathcal{P}}\right)}}\left(N(r, t ; n)-\frac{p(n)}{t}\right) q^{\ell_{t} n-\frac{\ell_{t}}{24}}
$$

is a weight $1 / 2$ weakly holomorphic modular form on $\Gamma_{1}\left(144 f_{t}^{2} \widetilde{\ell}_{t} \mathcal{P}^{4}\right)$. 
Proof. The non-holomorphic parts of the Maass forms in Theorem 9.5 have the property that their coefficients are supported on a fixed square class, numbers of the form $-\widetilde{\ell}_{t} n^{2}$. This square class is easily annihilated by taking linear combinations of quadratic twists. In particular, suppose that $\mathcal{P} \nmid 6 t$ is prime. For this prime $\mathcal{P}$, let

$$
g:=\sum_{v=1}^{\mathcal{P}-1}\left(\frac{v}{\mathcal{P}}\right) e^{\frac{2 \pi i v}{\mathcal{P}}}
$$

be the usual Gauss sum with respect to $\mathcal{P}$. Define the function $D\left(\frac{a}{t} ; z\right)_{\mathcal{P}}$ by

$$
D\left(\frac{a}{t} ; z\right)_{\mathcal{P}}:=\left.\frac{g}{\mathcal{P}} \sum_{v=1}^{\mathcal{P}-1}\left(\frac{v}{\mathcal{P}}\right) D\left(\frac{a}{t} ; z\right)\right|_{\frac{1}{2}}\left(\begin{array}{cc}
1 & -\frac{v}{\mathcal{P}} \\
0 & 1
\end{array}\right),
$$

where $\left.\right|_{\frac{1}{2}}$ is the usual slash operator (7.4). By construction, $D\left(\frac{a}{t} ; z\right)_{\mathcal{P}}$ is the $\mathcal{P}$-quadratic twist of $D\left(\frac{a}{t} ; z\right)$. In other words, the $n$th coefficient in the $q$-expansion of $D\left(\frac{a}{t} ; z\right)_{\mathcal{P}}$ is $\left(\frac{n}{\mathcal{P}}\right)$ times the $n$th coefficient of $D\left(\frac{a}{t} ; z\right)$. For the non-holomorphic part, this follows from the fact that the factors $\gamma(t, y ; n)$ appearing in (9.3) are fixed by the transformations in (9.4).

Generalizing classical facts about twists of modular forms, $D\left(\frac{a}{t} ; z\right)_{\mathcal{P}}$ is a Maass form of weight $1 / 2$ on $\Gamma_{1}\left(144 f_{t}^{2}{\widetilde{\ell_{t}}}^{2}\right)$. By $(9.3)$, it follows that

$$
D\left(\frac{a}{t} ; z\right)-\left(\frac{-\tilde{\ell}_{t}}{\mathcal{P}}\right) D\left(\frac{a}{t} ; z\right)_{\mathcal{P}}
$$

is a Maass form of weight $1 / 2$ on $\Gamma_{1}\left(144 f_{c}^{2} \widetilde{\ell}_{t} \mathcal{P}^{2}\right)$ with the property that its non-holomorphic part is supported on summands of the form $* q^{-\widetilde{\ell_{t}} \mathcal{P}^{2} n^{2}}$. These terms are annihilated by taking the $\mathcal{P}$-quadratic twist of this Maass form. Consequently, we obtain a weakly holomorphic modular form of weight $1 / 2$ on $\Gamma_{1}\left(144 f_{t}^{2} \widetilde{\ell}_{t} \mathcal{P}^{4}\right)$. Combining these observations with (9.2), we obtain the theorem.

REMARK 29. All of the results in this section continue to hold for even $t$ thanks to Theorem 6.3 and (8.13).

9.4. Implications for partition congruences. Here we apply Theorem 9.6 to shed light on the congruence properties of Dyson's functions $N(r, t ; n)$. We shall employ a method first used by the author in $[\mathbf{1 6 2}]$ in his work on $p(n)$ to show that Dyson's rank partition functions themselves uniformly satisfy Ramanujan-type congruences. Bringmann and the author have proved the following theorem (see Theorem 1.5 of [55], as well as [47]).

THEOREM 9.7. Let $t$ be a positive odd integer, and let $M$ be a positive integer coprime to 6 . Then there are infinitely many non-nested arithmetic progressions $A n+B$ such that for every $0 \leq r<t$ we have

$$
N(r, t ; A n+B) \equiv 0 \quad(\bmod M) .
$$


REMARK 30. Since we have

$$
p(A n+B)=\sum_{r=0}^{t-1} N(r, t ; A n+B),
$$

Theorem 9.7 provides a combinatorial decomposition of the partition function congruence

$$
p(A n+B) \equiv 0 \quad(\bmod M) .
$$

Sketch of the Proof of Theorem 9.7. For simplicity, we suppose that $M=Q^{j}$, where $5 \leq Q \nmid t$ is prime, and $j$ is a positive integer. The proof depends on Theorem 9.6, the observation that certain "sieved" partition rank generating functions are weakly holomorphic modular forms. This result then reduces the proof of Theorem 9.7 to the fact that any finite number of half-integral weight cusp forms with integer coefficients are annihilated, modulo a fixed prime power, by a positive proportion of half-integral weight Hecke operators.

To be precise, suppose that $f_{1}(z), f_{2}(z), \ldots, f_{s}(z)$ are half-integral weight cusp forms where

$$
f_{i}(z) \in S_{\lambda_{i}+\frac{1}{2}}\left(\Gamma_{1}\left(4 N_{i}\right)\right) \cap \mathcal{O}_{K}[[q]],
$$

and where $\mathcal{O}_{K}$ is the ring of integers of a fixed number field $K$. If $Q$ is prime and $j \geq 1$ is an integer, then the set of primes $L$ for which

$$
f_{i} \mid T_{\lambda_{i}+\frac{1}{2}}(L) \equiv 0 \quad\left(\bmod Q^{j}\right),
$$

for each $1 \leq i \leq s$, has positive Frobenius density. As before, here $T_{\lambda_{i}+\frac{1}{2}}(L)$ (see Section 7.5) denotes the usual $L^{2}$ index Hecke operator of weight $\lambda_{i}+\frac{1}{2}$.

Suppose that $\mathcal{P} \nmid 6 t Q$ is prime. By Theorem 9.6, for every $0 \leq r<t$

$$
\begin{aligned}
F(r, t, \mathcal{P} ; z) & =\sum_{n=1}^{\infty} a(r, t, \mathcal{P} ; n) q^{n} \\
& :=\sum_{\left(\frac{24 \ell_{t} n-\ell_{t}}{\mathcal{P}}\right)=-\left(\frac{-24 \widetilde{\ell_{t}}}{\mathcal{P}}\right)}\left(N(r, t ; n)-\frac{p(n)}{t}\right) q^{\ell_{t} n-\frac{\ell_{t}}{24}}
\end{aligned}
$$

is a weakly holomorphic modular form of weight $1 / 2$ on $\Gamma_{1}\left(144 f_{t}^{2} \widetilde{\ell}_{t} \mathcal{P}^{4}\right)$. Furthermore, by the work of Ahlgren and the author [3], it follows that

$$
P(t, \mathcal{P} ; z)=\sum_{n=1}^{\infty} p(t, \mathcal{P} ; n) q^{n}:=\sum_{\left(\frac{24 \ell_{t} n-\ell_{t}}{\mathcal{P}}\right)=-\left(\frac{-24 \tilde{\ell_{t}}}{\mathcal{P}}\right)} p(n) q^{\ell_{t} n-\frac{\ell_{t}}{24}}
$$

is a weakly holomorphic modular form of weight $-1 / 2$ on $\Gamma_{1}\left(576 \tilde{\ell}_{t} \mathcal{P}^{4}\right)$. In particular, all of these forms are modular with respect to $\Gamma_{1}\left(576 f_{t}^{2} \widetilde{\ell}_{t} \mathcal{P}^{4}\right)$. 
Since $Q \nmid 576 f_{t}^{2} \widetilde{\ell}_{t} \mathcal{P}^{4}$, a result of Treneer (see Theorem 3.1 of $[\mathbf{2 0 1}]$ ), generalizing earlier observations of Ahlgren and the author [3, 162], implies that there is a sufficiently large integer $m$ for which

$$
\sum_{Q \nmid n} a\left(r, t, \mathcal{P} ; Q^{m} n\right) q^{n}
$$

for all $0 \leq r<t$, and

$$
\sum_{Q \nmid n} p\left(t, \mathcal{P} ; Q^{m} n\right) q^{n}
$$

are all congruent modulo $Q^{j}$ to forms in the graded ring of half-integral weight cusp forms with algebraic integer coefficients on $\Gamma_{1}\left(576 f_{t}^{2} \widetilde{\ell_{t}} \mathcal{P}^{4} Q^{2}\right)$.

The system of simultaneous congruences (9.6), in the case of these forms, guarantees that a positive proportion of primes $L$ have the property that these forms modulo $Q^{j}$ are annihilated by the index $L^{2}$ half-integral weight Hecke operators. Theorem 9.7 now follows mutatis mutandis as in the proof of Theorem 1 of [162]. One merely interprets this annihilation, using (7.14), in terms of the $N(r, t ; n)$.

Two remarks.

1) The simultaneous system (9.6) of congruences follows from a straightforward generalization of a classical observation of Serre (see Section 6 of [188]). 2) Treneer states her result for weakly holomorphic modular forms on $\Gamma_{0}(4 N)$ with Nebentypus. We are using a straightforward extension of her result to $\Gamma_{1}(4 N)$.

9.5. Related recent works. Recently there have been many works on the relationship between Dyson's rank and the Andrews-Garvan crank [25]. Several years ago, Atkin and Garvan [30] discovered a striking partial differential equation which related the rank and crank generating functions. Their work then inspired the recent investigation of Andrews on Durfee symbols and higher partition statistics [19].

Armed with the new understanding of partition ranks in terms of harmonic Maass forms, much light has been shed recently on these results from the perspective of modular forms and Maass forms. In this direction there are papers by Bringmann, Garvan, Lovejoy, Mahlburg, and Osburn $[4 \mathbf{4 8}, \mathbf{5 0}, \mathbf{5 2}]$, in which theorems concerning the automorphic properties of partition generating functions, asymptotics, and congruences are obtained for various partition statistics such as those arising in the work of Andrews [19] on rank moments. In a recent paper, Bringmann and Zwegers [60] investigate further the phenomenon of the the Atkin-Garvan "crank-rank" PDE from the perspective of non-holomorphic Jacobi forms.

In another recent development, M. Monks, an undergraduate at MIT, has discovered a deep connection between the power series $R\left( \pm i ; q^{-1}\right)$, some false theta functions of Rogers, and the generating function for Dyson's ranks restricted to partitions into distinct parts. Her work [156] allows 
one to determine the behavior of the holomorphic part of the Maass form $D( \pm 1 / 4 ; z)$ in Theorem 8.6 for complex $z$ in the lower half of the complex plane.

\section{Eulerian series as modular forms}

Eulerian series are combinatorial formal power series which are constructed from basic hypergeometric series. As described in Section 2.3, there are famous examples of Eulerian series which essentially are modular forms. For example, we have the celebrated Rogers-Ramanujan identities

$$
\begin{aligned}
& \prod_{n=0}^{\infty} \frac{1}{\left(1-q^{5 n+1}\right)\left(1-q^{5 n+4}\right)}=1+\sum_{n=1}^{\infty} \frac{q^{n^{2}}}{(1-q)\left(1-q^{2}\right) \cdots\left(1-q^{n}\right)}, \\
& \prod_{n=0}^{\infty} \frac{1}{\left(1-q^{5 n+2}\right)\left(1-q^{5 n+3}\right)}=1+\sum_{n=1}^{\infty} \frac{q^{n^{2}+n}}{(1-q)\left(1-q^{2}\right) \cdots\left(1-q^{n}\right)} .
\end{aligned}
$$

These identities provide series expansions of infinite products which correspond to weight 0 modular forms. As another example, we have Theorem 2.2, which asserts that the partition number generating function satisfies

$$
\prod_{n=1}^{\infty} \frac{1}{1-q^{n}}=1+\sum_{n=1}^{\infty} \frac{q^{n^{2}}}{(1-q)^{2}\left(1-q^{2}\right)^{2} \cdots\left(1-q^{n}\right)^{2}} .
$$

Since this series is essentially the reciprocal of Dedekind's weight $1 / 2$ modular form, this provides another example of an Eulerian series which is a modular form.

The literature on such identities is extensive, and the pursuit of further identities and their interpretations remains an active area of research largely due to applications in combinatorics, Lie theory, number theory and physics (for example, see $[\mathbf{1 3}],[\mathbf{1 2 9}]$, and [145] to name a few).

Here we illustrate how one may easily obtain modular forms from Eulerian series by forcing the cancellation of non-holomorphic parts of harmonic Maass forms. To this end, we again consider Dyson's generating function $R(w ; q)$

$$
R(w ; q):=1+\sum_{n=1}^{\infty} \sum_{m=-\infty}^{\infty} N(m, n) w^{m} q^{n}=1+\sum_{n=1}^{\infty} \frac{q^{n^{2}}}{(w q ; q)_{n}\left(w^{-1} q ; q\right)_{n}},
$$

and the functions $N(r, t ; n)$.

By conjugating Ferrers boards, we have the trivial relation

$$
N(r, t ; n)=N(t-r, t ; n) .
$$

However, these functions also satisfy [31] some highly non-trivial sporadic identities such as

$$
N(1,7 ; 7 n+1)=N(2,7 ; 7 n+1)=N(3,7 ; 7 n+1) .
$$


Atkin and Swinnerton-Dyer [31] proved some surprising further identities such as (see also (5.19) of $[\mathbf{1 1 9}]$ )

$$
-\frac{\left(q ; q^{7}\right)_{\infty}^{2}\left(q^{6} ; q^{7}\right)_{\infty}^{2}\left(q^{7} ; q^{7}\right)_{\infty}^{2}}{(q ; q)_{\infty}}=\sum_{n=0}^{\infty}(N(0,7 ; 7 n+6)-N(1,7 ; 7 n+6)) q^{n}
$$

This identity expresses a weight $1 / 2$ modular form as a linear combination of Eulerian series.

This example is a special case of a much more general phenomenon which gives highly nontrivial relationships between Dyson's partition rank functions. Generalizing it, Bringmann, Rhoades, and the author [57] used the theory of harmonic Maass forms to obtain several infinite families of modular forms as Eulerian series. Here we present examples which arise directly from Theorem 9.5.

Theorem 10.1. Suppose that $t \geq 5$ is prime, $0 \leq r_{1}, r_{2}<t$ and $0 \leq d<$ t. Then the following are true:

(1) If $\left(\frac{1-24 d}{t}\right)=-1$, then

$$
\sum_{n=0}^{\infty}\left(N\left(r_{1}, t ; t n+d\right)-N\left(r_{2}, t ; t n+d\right)\right) q^{24(t n+d)-1}
$$

is a weight $1 / 2$ weakly holomorphic modular form on $\Gamma_{1}\left(576 t^{6}\right)$.

(2) Suppose that $\left(\frac{1-24 d}{t}\right)=1$. If $r_{1}, r_{2} \not \equiv \frac{1}{2}( \pm 1 \pm \alpha)(\bmod t)$, where $\alpha$ is any integer for which $0 \leq \alpha<2 t$ and $1-24 d \equiv \alpha^{2}(\bmod 2 t)$, then

$$
\sum_{n=0}^{\infty}\left(N\left(r_{1}, t ; t n+d\right)-N\left(r_{2}, t ; t n+d\right)\right) q^{24(t n+d)-1}
$$

is a weight 1/2 weakly holomorphic modular form on $\Gamma_{1}\left(576 t^{6}\right)$.

Remark 31. Example (10.3) is the $t=7$ and $d=6$ case of Theorem 10.1 (2). In this case, the only choices of $r_{1}$ and $r_{2}$ satisfying the hypotheses are 0,1 , and 6 . Since $N(1,7 ; n)=N(6,7 ; n),(10.3)$ is the only nontrivial example of Theorem 10.1 (2) in this case. The proof of the theorem will show, for all other pairs of $r_{1}$ and $r_{2}$ (apart from the trivial examples such as those arising from (10.1)), that

$$
\sum_{n=0}^{\infty}\left(N\left(r_{1}, t ; t n+d\right)-N\left(r_{2}, t ; t n+d\right)\right) q^{24(t n+d)-1}
$$

is not a weakly holomorphic modular form. In other words, the corresponding Maass forms turn out to have non-trivial non-holomorphic parts.

Remark 32. The "Mock theta Conjectures" (see Section 4), which were proved by Hickerson, are instances of pairs of harmonic Maass forms which 
differ by a weakly holomorphic modular form. Using arguments along the lines of the proofs in this section, A. Folsom has provided [94] a "Maass form" proof of these conjectures.

REMARK 33. In a paper, S.-Y. Kang [132] has further developed the relationship between basic hypergeometric series and nonholomorphic Jacobi forms using the work of Zwegers. In particular, she gives (see Theorems 1.2 and 1.3 of [132]) two explicit families of weight $1 / 2$ modular forms, described in terms of theta functions and Dedekind's eta-function, which are sums of basic hypergeometric series.

Theorem 10.1 gives modular forms as differences of the generating functions for the functions $N(r, t ; t n+d)$. There are similar theorems where the rank modulus $t$ is independent of the modulus of the arithmetic progression of the sizes of the partitions. As before, for integers $0<a<t$, let $f_{t}:=\frac{2 t}{\operatorname{gcd}(t, 6)}, \ell_{t}:=\operatorname{lcm}\left(2 t^{2}, 24\right)$, and let $\widetilde{\ell}_{t}:=\ell_{t} / 24$. We then have the following theorem which is contained in [57].

Theorem 10.2. Suppose that $t>1$ is an odd integer. If $0 \leq r_{1}, r_{2}<t$ are integers, and $\mathcal{P} \nmid 6 t$ is prime, then

$$
\begin{aligned}
& \sum_{n \geq 1}\left(N\left(r_{1}, t ; n\right)-N\left(r_{2}, t ; n\right)\right) q^{\ell_{t} n-\frac{\ell_{t}}{24}} \\
& \left(\frac{24 \ell_{t} n-\ell_{t}}{\mathcal{P}}\right)=-\left(\frac{-24 \tilde{\mathcal{P}}}{\mathcal{P}}\right)
\end{aligned}
$$

is a weight $1 / 2$ weakly holomorphic modular form on $\Gamma_{1}\left(144 f_{t}^{2} \widetilde{\ell}_{t} \mathcal{P}^{4}\right)$.

Theorems 10.1 and 10.2 depend on Theorem 9.5. However, one may obtain many further results of this type by making use of results which are analogous to Theorem 9.5. This is done in [57], where we obtain several further infinite families of Eulerian modular forms. These families give rise to further identities such as

$$
2 \frac{\left(q^{4} ; q^{4}\right)_{\infty}^{5}}{\left(q^{2} ; q^{2}\right)_{\infty}^{4}}=\sum_{n \geq 0} \frac{q^{n(n+1)}\left(-q^{2} ; q^{2}\right)_{n}}{\left(q ; q^{2}\right)_{n+1}^{2}}+\sum_{n \geq 0} \frac{q^{n(n+1)}\left(-q^{2} ; q^{2}\right)_{n}}{\left(-q ; q^{2}\right)_{n+1}^{2}} .
$$

10.1. Proofs of Theorems $\mathbf{1 0 . 1}$ and 10.2. Theorem 10.2 follows immediately from Theorem 9.6 since the $p(n) / t$ summands cancel when taking the difference of the relevant generating functions.

Now we turn to the proof of Theorem 10.1. By Theorem 9.5, for any $0 \leq r<t$ we have

$$
\sum_{n=0}^{\infty}\left(N(r, t ; n)-\frac{p(n)}{t}\right) q^{24 t^{2} n-t^{2}}+\sum_{n \in \mathbb{Z}} \widetilde{A}(r, t, n) \cdot \gamma(t, y ; n) q^{-t^{2} n^{2}}
$$


is a weight $1 / 2$ harmonic Maass form on $\Gamma_{1}\left(576 t^{4}\right)$. Here $\widetilde{A}(r, t, n)$ is a complex number given by

$$
\widetilde{A}(r, t, n)=i \sqrt{8} \sum_{\substack{m \\ 6 m+1 \equiv n(\bmod 2 t)}} A(r, t, m),
$$

where $\gamma(t, y ; n)$ and $A(r, t ; m)$ are defined in Theorem 9.5. Applying the Atkin $U\left(t^{2}\right)$ operator, we have, by a straightforward generalization of Proposition 1.5 of [192], that

$$
\mathfrak{R}(r, t ; z):=\sum_{n=0}^{\infty}\left(N(r, t ; n)-\frac{p(n)}{t}\right) q^{24 n-1}+\sum_{n \in \mathbb{Z}} \widetilde{A}(r, t, n) \cdot \gamma(t, y ; n) q^{-n^{2}}
$$

is a weight $1 / 2$ harmonic Maass form on $\Gamma_{1}\left(576 t^{4}\right)$.

Now we prove Theorem 10.1 (1). By a straightforward generalization of the classical argument on twists of modular forms (for example, see Proposition 22 of $[\mathbf{1 3 4}])$, the quadratic twist of $\mathfrak{R}(r, t ; z)$ by $(\dot{\bar{t}})$, say $\mathfrak{R}(r, t ; z)_{t}$, is a weight $1 / 2$ harmonic Maass form on $\Gamma_{1}\left(576 t^{6}\right)$. In particular, $\left(\frac{-1}{t}\right) \mathfrak{R}(r, t ; z)_{t}$ has an expansion of the form

$$
\begin{aligned}
\left(\frac{-1}{t}\right) \mathfrak{R}(r, t ; z)_{t}= & \sum_{n=0}^{\infty}\left(\frac{1-24 n}{t}\right)\left(N(r, t ; n)-\frac{p(n)}{t}\right) q^{24 n-1} \\
& +\sum_{\substack{n \in \mathbb{Z} \\
t \nmid n}} \widetilde{A}(r, t, n) \cdot \gamma(t, y ; n) q^{-n^{2}} .
\end{aligned}
$$

We find that $\mathfrak{R}(r, t ; z)-\left(\frac{-1}{t}\right) \mathfrak{R}(r, t ; z)_{t}$ is on $\Gamma_{1}\left(576 t^{6}\right)$, and its non-holomorphic part is supported on terms of the form $q^{-t^{2} n^{2}}$. By taking the quadratic twist of this form again by $(\dot{\bar{t}})$, to annihilate these non-holomorphic terms, one then finds that

$$
\sum_{\substack{n \geq 0,\left(\frac{1-24 n}{t}\right)=-1}}\left(N(r, t ; n)-\frac{p(n)}{t}\right) q^{24 n-1}
$$

is weight $1 / 2$ weakly holomorphic modular form. Using the orthogonality of Dirichlet characters modulo $t$, and facts about twists again, it follows that

$$
\sum_{n=0}^{\infty}\left(N(r, t ; t n+d)-\frac{p(t n+d)}{t}\right) q^{24(t n+d)-1}
$$

is a weight $1 / 2$ weakly holomorphic modular form. Theorem 10.1 (1) follows by taking the difference of these forms when $r=r_{1}$ and $r_{2}$. Since taking twists of twists can be viewed as a single twist by the trivial character, we find that the resulting form is on $\Gamma_{1}\left(576 t^{6}\right)$.

Now we turn to the proof of Theorem 10.1 (2). Here we argue directly with (9.1) and (10.5). Using the theory of twists of Maass forms again, we see that the restriction of $\mathfrak{R}\left(r_{1}, t ; z\right)-\mathfrak{R}\left(r_{2}, t ; z\right)$ to forms whose holomorphic 
parts are supported on exponents of the form $24(t n+d)-1$, is a weight $1 / 2$ harmonic Maass form on $\Gamma_{1}\left(576 t^{6}\right)$.

It suffices to show that the non-holomorphic part of this form is zero under the given hypotheses on $r_{1}$ and $r_{2}$. By (10.5), one sees that the nonholomorphic part is supported on terms of the form $q^{-n^{2}}$. By construction, these $n$ satisfy $n \equiv \alpha(\bmod 2 t)$, for some $0 \leq \alpha<2 t$ with $1-24 d \equiv \alpha^{2}$ $(\bmod 2 t)$. Therefore, by $(9.1)$ and $(10.5)$, it suffices to show that

$$
A\left(r_{1}, t, m\right)-A\left(r_{2}, t, m\right)=0,
$$

when $6 m+1 \equiv \alpha(\bmod 12 t)$. By $(9.1)$, we have

$$
A\left(r_{1}, t, m\right)-A\left(r_{2}, t, m\right)=(-1)^{m} \sum_{j=1}^{t-1}\left(\zeta_{t}^{-r_{1} j}-\zeta_{t}^{-r_{2} j}\right) \sin \left(\frac{\pi j}{t}\right) \sin \left(\frac{\pi j \alpha}{t}\right) .
$$

Since $\sin (x)=\frac{1}{2 i}\left(e^{i x}-e^{-i x}\right)$, we have that

$$
\begin{aligned}
& \sum_{j=1}^{t-1}\left(e^{\frac{\pi i j}{t}\left(1-2 r_{1}+\alpha\right)}-e^{\frac{\pi i j}{t}\left(1-2 r_{1}-\alpha\right)}-e^{\frac{\pi i j}{t}\left(-1-2 r_{1}+\alpha\right)}+e^{\frac{\pi i j}{t}\left(-1-2 r_{1}-\alpha\right)}\right. \\
& \left.-e^{\frac{\pi i j}{t}\left(1-2 r_{2}+\alpha\right)}+e^{\frac{\pi i j}{t}\left(1-2 r_{2}-\alpha\right)}+e^{\frac{\pi i j}{t}\left(-1-2 r_{2}+\alpha\right)}-e^{\frac{\pi i j}{t}\left(-1-2 r_{2}-\alpha\right)}\right)=0 .
\end{aligned}
$$

This follows since $\pm 1-2 r_{i} \pm \alpha$, for $i=1$ and 2 , are even and coprime to $t$.

\section{Exact formulas}

Armed with the theory of harmonic Maass forms, one may obtain exact formulas for the coefficients of generating functions which turn out to be holomorphic parts of such forms. By employing the method of Poincaré series as described in Section 8.3, one obtains such results. Here we first describe the recent resolution of the Andrews-Dragonette Conjecture, and then we give exact formulas for generic harmonic Maass forms with weight $\leq 1 / 2$.

11.1. The Andrews-Dragonette conjecture. Rademacher famously employed the modularity of the generating function for $p(n)$ to perfect the Hardy-Ramanujan asymptotic formula

$$
p(n) \sim \frac{1}{4 n \sqrt{3}} \cdot e^{\pi \sqrt{2 n / 3}}
$$

to obtain his "exact" formula. To state his formula, let $I_{s}(x)$ be the usual $I$-Bessel function of order $s$. Furthermore, if $k \geq 1$ and $n$ are integers, then let

$$
A_{k}(n):=\frac{1}{2} \sqrt{\frac{k}{12}} \sum_{\substack{x \\ x^{2} \equiv-24 n+1 \quad(\bmod 24 k)}} \chi_{12}(x) \cdot e\left(\frac{x}{12 k}\right)
$$


K. ONO

where the sum runs over the residue classes modulo $24 k$, and where

$$
\chi_{12}(x):=\left(\frac{12}{x}\right) \text {. }
$$

If $n$ is a positive integer, then Rademacher's formula reads [168]

$$
p(n)=2 \pi(24 n-1)^{-\frac{3}{4}} \sum_{k=1}^{\infty} \frac{A_{k}(n)}{k} \cdot I_{\frac{3}{2}}\left(\frac{\pi \sqrt{24 n-1}}{6 k}\right) .
$$

Here we address the problem of obtaining such exact formulas for $N_{e}(n)$ (resp. $N_{o}(n)$ ), the number of partitions of $n$ with even (resp. odd) rank. To obtain these results, we begin with the simple observation that

$$
\begin{aligned}
f(q)=R(-1 ; q) & =1+\sum_{n=1}^{\infty}\left(N_{e}(n)-N_{o}(n)\right) q^{n} \\
& =1+\sum_{n=1}^{\infty} \frac{q^{n^{2}}}{(1+q)^{2}\left(1+q^{2}\right)^{2} \cdots\left(1+q^{n}\right)^{2}} .
\end{aligned}
$$

In view of (11.4) and (11.5), since

$$
p(n)=N_{e}(n)+N_{o}(n),
$$

our task is reduced to the problem of deriving exact formulas for the coefficients $\alpha(n)$ of the mock theta function

$$
f(q)=R(-1 ; q)=1+\sum_{n=1}^{\infty} \alpha(n) q^{n} .
$$

The problem of estimating the coefficients $\alpha(n)$ has a long history, one which even precedes Dyson's definition of partition ranks. Indeed, Ramanujan's last letter to Hardy already includes the claim that

$$
\alpha(n)=(-1)^{n-1} \frac{\exp \left(\pi \sqrt{\frac{n}{6}-\frac{1}{144}}\right)}{2 \sqrt{n-\frac{1}{24}}}+O\left(\frac{\exp \left(\frac{1}{2} \pi \sqrt{\frac{n}{6}-\frac{1}{144}}\right)}{\sqrt{n-\frac{1}{24}}}\right) .
$$

Typical of his writings, Ramanujan offered no proof of this claim. Dragonette proved this claim in her 1951 Ph.D. thesis [85], and Andrews [7] subsequently improved upon Dragonette's work, and he proved ${ }^{16}$ that

$$
\begin{aligned}
\alpha(n)= & \pi(24 n-1)^{-\frac{1}{4}} \sum_{k=1}^{[\sqrt{n}]} \frac{(-1)^{\rfloor^{\left.\frac{k+1}{2}\right\rfloor}} A_{2 k}\left(n-\frac{k\left(1+(-1)^{k}\right)}{4}\right)}{k} \\
& \cdot I_{\frac{1}{2}}\left(\frac{\pi \sqrt{24 n-1}}{12 k}\right)+O\left(n^{\epsilon}\right) .
\end{aligned}
$$

${ }^{16}$ This is a reformulation of Theorem 5.1 of $[7]$ using the identity $I_{\frac{1}{2}}(z)=\left(\frac{2}{\pi z}\right)^{\frac{1}{2}}$. $\sinh (z)$. 
This result falls short of the problem of obtaining an exact formula for $\alpha(n)$. Andrews and Dragonette formulated the following conjecture (see page 456 of [7], and Section 5 of [17]) for the coefficients $\alpha(n)$.

Conjecture. (Andrews-Dragonette)

If $n$ is a positive integer, then

$\alpha(n)=\pi(24 n-1)^{-\frac{1}{4}} \sum_{k=1}^{\infty} \frac{(-1)^{\left\lfloor\frac{k+1}{2}\right\rfloor} A_{2 k}\left(n-\frac{k\left(1+(-1)^{k}\right)}{4}\right)}{k} \cdot I_{\frac{1}{2}}\left(\frac{\pi \sqrt{24 n-1}}{12 k}\right)$.

Bringmann and the author have proved [53] the following theorem.

Theorem 11.1. The Andrews-Dragonette Conjecture is true.

Remark 34. In her Ph.D. thesis (see $[\mathbf{9 7}, \mathbf{9 8}]$ ), S. Garthwaite generalized the proof of Theorem 11.1, and she obtained exact formulas for the coefficients of Ramanujan's mock theta function $\omega(q)$. These results will be further generalized in the next subsection.

Sketch of the Proof of Theorem 11.1. By Theorem 6.1, we have that $D\left(\frac{1}{2} ; z\right)$ (see $(8.12)$ ) is a harmonic weight $1 / 2$ Maass form on $\Gamma_{0}(144)$ with Nebentypus character $\chi_{12}=\left(\frac{12}{.}\right)$.

The idea behind the proof is simple. We shall construct a MaassPoincaré series which we shall show equals $D\left(\frac{1}{2} ; z\right)$. The method is analogous to material in Section 8.3. The proof of the conjecture then follows from the fact that the formulas in the Andrews-Dragonette Conjecture give the coefficients of this Maass-Poincaré series.

Suppose that $k \in \frac{1}{2}+\mathbb{Z}$. We define a class of Poincaré series $P_{k}(s ; z)$. For matrices $\left(\begin{array}{ll}a & b \\ c & d\end{array}\right) \in \Gamma_{0}(2)$, with $c \geq 0$, define the character $\chi(\cdot)$ by

$\chi\left(\left(\begin{array}{ll}a & b \\ c & d\end{array}\right)\right):= \begin{cases}e\left(-\frac{b}{24}\right) & \text { if } c=0, \\ i^{-1 / 2}(-1)^{\frac{1}{2}(c+a d+1)} e\left(-\frac{a+d}{24 c}-\frac{a}{4}+\frac{3 d c}{8}\right) \cdot \omega_{-d, c}^{-1} & \text { if } c>0,\end{cases}$

where

$$
\omega_{d, c}:=e^{\pi i s(d, c)} .
$$

Here $s(d, c)$ denotes the classical Dedekind sum.

Throughout, let $z=x+i y$, and for $s \in \mathbb{C}, k \in \frac{1}{2}+\mathbb{Z}$, and $y \in \mathbb{R} \backslash\{0\}$, and let

$$
\mathcal{M}_{s}(y):=|y|^{-\frac{k}{2}} M_{\frac{k}{2} \operatorname{sgn}(y), s-\frac{1}{2}}(|y|),
$$

where $M_{\nu, \mu}(z)$ again is the $M$-Whittaker function. Furthermore, let

$$
\varphi_{s, k}(z):=\mathcal{M}_{s}\left(-\frac{\pi y}{6}\right) e\left(-\frac{x}{24}\right) .
$$

Using this notation, define the Poincaré series $P_{k}(s ; z)$ by

$$
P_{k}(s ; z):=\frac{2}{\sqrt{\pi}} \sum_{M \in \Gamma_{\infty} \backslash \Gamma_{0}(2)} \chi(M)^{-1}(c z+d)^{-k} \varphi_{s, k}(M z) .
$$


Here $\Gamma_{\infty}$ again is the subgroup of translations in $\mathrm{SL}_{2}(\mathbb{Z})$.

The defining series is absolutely convergent for $P_{k}\left(1-\frac{k}{2} ; z\right)$ for $k<$ $1 / 2$, and is conditionally convergent when $k=1 / 2$. We are interested in $P_{\frac{1}{2}}\left(\frac{3}{4} ; z\right)$, which we define by analytically continuing the Fourier expansion. This argument is not straightforward (see Theorem 3.2 and Corollary 4.2 of [53]). Thanks to the properties of $M_{\nu, \mu}$, we find that $P_{\frac{1}{2}}\left(\frac{3}{4} ; 24 z\right)$ is a Maass form of weight $1 / 2$ for $\Gamma_{0}(144)$ with Nebentypus $\chi_{12}$.

A long calculation gives the following Fourier expansion

$$
\begin{aligned}
P_{\frac{1}{2}}\left(\frac{3}{4} ; z\right)= & \left(1-\pi^{-\frac{1}{2}} \cdot \Gamma\left(\frac{1}{2}, \frac{\pi y}{6}\right)\right) \cdot q^{-\frac{1}{24}} \\
& +\sum_{n=-\infty}^{0} \gamma_{y}(n) q^{n-\frac{1}{24}}+\sum_{n=1}^{\infty} \beta(n) q^{n-\frac{1}{24}}
\end{aligned}
$$

where for positive integers $n$ we have

$\beta(n)=\pi(24 n-1)^{-\frac{1}{4}} \sum_{k=1}^{\infty} \frac{(-1)^{\left\lfloor\frac{k+1}{2}\right\rfloor} A_{2 k}\left(n-\frac{k\left(1+(-1)^{k}\right)}{4}\right)}{k} \cdot I_{\frac{1}{2}}\left(\frac{\pi \sqrt{24 n-1}}{12 k}\right)$.

The Poincaré series $P_{\frac{1}{2}}\left(\frac{3}{4} ; z\right)$ was defined so that (11.12) coincides with the conjectured expressions for the coefficients $\alpha(n)$.

For convenience, we let

$$
P(z):=P_{\frac{1}{2}}\left(\frac{3}{4} ; 24 z\right) .
$$

Canonically decompose $P(z)$ into a non-holomorphic and a holomorphic part

$$
P(z)=P^{-}(z)+P^{+}(z) .
$$

In particular, we have that

$$
P^{+}(z)=q^{-1}+\sum_{n=1}^{\infty} \beta(n) q^{24 n-1} .
$$

Since $P(z)$ and $D\left(\frac{1}{2} ; z\right)$ are Maass forms of weight $1 / 2$ for $\Gamma_{0}(144)$ with Nebentypus $\chi_{12},(11.11)$ and (11.12) imply that the proof of the conjecture reduces to proving that these forms are equal. This conclusion is obtained after a lengthy and somewhat complicated argument.

11.2. Exact formulas for harmonic Maass forms with weight $\leq 1 / 2$. Generalizing the results of the previous section, Bringmann and the author have obtained exact formulas for the coefficients of the holomorphic parts of harmonic Maass forms with weight $2-k \leq 1 / 2$ [59]. Suppose that $f$ is in $H_{2-k}(N, \chi)$, the space of weight $2-k$ harmonic Maass forms on $\Gamma_{0}(N)$ 
with Nebentypus character $\chi$, where we assume that $\frac{3}{2} \leq k \in \frac{1}{2} \mathbb{Z}$. As usual, we denote its Fourier expansion by

$$
f(z)=\sum_{n \gg-\infty} c_{f}^{+}(n) q^{n}+\sum_{n<0} c_{f}^{-}(n) \Gamma(k-1,4 \pi|n| y) q^{n} .
$$

It is our objective to determine exact formulas for the coefficients $c_{f}^{+}(n)$ of the holomorphic part of $f$.

We now define the functions which are required for these exact formulas. Throughout, we let $k \in \frac{1}{2} \mathbb{Z}$, and we let $\chi$ be a Dirichlet character modulo $N$, where $4 \mid N$ whenever $k \in \frac{1}{2} \mathbb{Z} \backslash \mathbb{Z}$. Using this character, for a matrix $M=\left(\begin{array}{ll}a & b \\ c & d\end{array}\right) \in \Gamma_{0}(N)$, we let

$$
\Psi_{k}(M):= \begin{cases}\chi(d) & \text { if } k \in \mathbb{Z} \\ \chi(d)\left(\frac{c}{d}\right) \epsilon_{d}^{2 k} & \text { if } k \in \frac{1}{2} \mathbb{Z} \backslash \mathbb{Z}\end{cases}
$$

where $\epsilon_{d}$ is defined by (7.2), and where $\left(\frac{c}{d}\right)$ is the usual extended Legendre symbol. In addition, if $T=\left(\begin{array}{ll}a & b \\ c & d\end{array}\right) \in \mathrm{SL}_{2}(\mathbb{Z})$, then we let

$$
\mu(T ; z):=(c z+d)^{2-k} .
$$

Moreover, for pairs of matrices $S, T \in \mathrm{SL}_{2}(\mathbb{Z})$, we then let

$$
\sigma(T, S):=\frac{\mu(T ; S z) \mu(S ; z)}{\mu(T S ; z)} .
$$

Using this notation, we now define certain generic Kloosterman sums which are naturally associated with cusps of $\Gamma_{0}(N)$.

Suppose that $\rho=\frac{a_{\rho}}{c_{\rho}}=L^{-1} \infty,\left(L \in \mathrm{SL}_{2}(\mathbb{Z})\right)$ is a cusp of $\Gamma_{0}(N)$ with $c_{\rho} \mid N$ and $\operatorname{gcd}\left(a_{\rho}, N\right)=1$. Let $t_{\rho}$ and $\kappa_{\rho}$ be the cusp width and parameter of $\rho$ with respect to $\Gamma_{0}(N)$ (see 11.21). Suppose that $c>0$ with $c_{\rho} \mid c$ and $\frac{N}{c_{\rho}} \nmid c$. Then for integers $n$ and $m$ we have the Kloosterman sum

$$
\begin{aligned}
& K_{c}(2-k, \rho, \chi, m, n) \\
& :=\sum_{\substack{0<d<c \\
0<a<c t \\
a_{\rho} a \equiv-\frac{c}{c_{\rho}}\left(\bmod \frac{N}{c_{\rho}}\right) \\
(a d, c)=1}} \frac{\sigma\left(L^{-1}, S\right)}{\Psi_{k}\left(L^{-1} S\right)} \cdot \exp \left(\frac{2 \pi i}{c}\left(\frac{\left(m+\kappa_{\rho}\right) a}{t_{\rho}}+n d\right)\right),
\end{aligned}
$$

where $S:=\left(\begin{array}{ll}a & b \\ c & d\end{array}\right) \in \mathrm{SL}_{2}(\mathbb{Z})$ is the unique matrix defined using the integers $a, c$, and $d$. Using properties of $\sigma$ and $\Psi_{k}$, one can easily show that (11.19) is well-defined.

For convenience, we let $\mathcal{S}_{N}$ be a subset of $\mathrm{SL}_{2}(\mathbb{Z})$ with the property that

$$
\left\{S^{-1} \infty: S \in \mathcal{S}_{N}\right\}
$$


represents the set of inequivalent cusps of $\Gamma_{0}(N)$. For $M=\left(\begin{array}{ll}a & b \\ c & d\end{array}\right) \in \mathrm{SL}_{2}(\mathbb{Z})$, we define

$$
f_{M}(z):=(c z+d)^{k-2} f\left(\frac{a z+b}{c z+d}\right),
$$

where $\sqrt{z}$ is the principal branch of the holomorphic square root. Using this notation, we have the Fourier expansion of a form $f$ at a cusp $\rho$. More precisely, if $L \in \mathcal{S}_{N}$ with $\rho=L^{-1} \infty$, then we have

$$
f_{\rho}(z)=\sum_{n \in \mathbb{Z}} a_{\rho}^{+}(n) q^{\frac{n+\kappa_{\rho}}{t_{\rho}}}+f_{\rho}^{-}(\tau) .
$$

We define the principal part of $f$ at $\rho$ by

$$
P_{f, \rho}(z):=\sum_{m+\kappa_{\rho}<0} a_{\rho}^{+}(m) q^{\frac{m+\kappa_{\rho}}{t_{\rho}}} .
$$

We shall use the principal parts of a form $f$ to determine our exact formulas. To this end, we identify, for each cusp $\rho$, its contribution to the exact formula. To make this precise, let $M=L^{-1}$ and $\mu=L \infty$. For positive $n$, we then define

$$
\begin{aligned}
& \mathcal{A}(N, 2-k, \chi, \rho, m, c ; n):=-\frac{i^{k} 2 \pi}{t_{\mu}}\left|\frac{\left(-m+\kappa_{\mu}\right)}{t_{\mu} n}\right|^{\frac{k-1}{2}} \\
& \quad \times \sum_{\substack{c>0 \\
c_{\mu} \mid c, \frac{N}{c \mu} \nmid c}} \frac{K_{c}(2-k, \mu, \chi,-m,-n)}{c} \cdot I_{k-1}\left(\frac{4 \pi}{c} \sqrt{\frac{n\left|-m+\kappa_{\mu}\right|}{t_{\mu}}}\right) .
\end{aligned}
$$

Here $t_{\mu}$ and $\kappa_{\mu}$ are the cusp parameters for $\mu$ as in the notation above.

Using this notation, we define the order $\mathcal{N}$ Kloosterman approximation of $c_{f}^{+}(n)$ by

$$
\mathcal{C}(f, \mathcal{N} ; n):=\sum_{L \in \mathcal{S}_{N}} \sum_{m+\kappa_{\rho}<0} a_{\rho}^{+}(m) \sum_{c=1}^{\mathcal{N}} \mathcal{A}(N, 2-k, \chi, \rho, m, c ; n) .
$$

Moreover, we define $\mathcal{C}(f, \infty ; n)$ in the obvious way.

REMARK 35. We stress again that $L$ and $\rho$ are related (throughout this section) by the formula $\rho=L^{-1} \infty$.

THEOREM 11.2. If $f \in H_{2-k}(N, \chi)$ with $2 \leq k \in \frac{1}{2} \mathbb{Z}$, then for positive $n$ we have

$$
c_{f}^{+}(n)=\mathcal{C}(f, \infty ; n) .
$$

REMARK 36. Using the asymptotic behavior of $I$-Bessel functions, an inspection of the principal parts of $f$ gives a minimal $\mathcal{N}$ for which

$$
\mathcal{C}(f, \mathcal{N} ; n) \sim c_{f}^{+}(n) .
$$


REMARK 37. Theorem 11.2 includes the classic results of Rademacher and Zuckerman $[\mathbf{1 6 9}, \mathbf{1 7 0}, \mathbf{2 1 6}, \mathbf{2 1 7}]$ in the very special case of those $f \in H_{2-k}(N, \chi)$ for which $2-k<0$ and $f^{-}=0$. It also recovers some results of Bruinier, Hejhal, and Niebur $[62,117,160,161]$ for harmonic Maass forms of non-positive weight. Recent work by Bruinier and Strömberg [69] addresses the problem of efficiently computing coefficients of harmonic Maass forms.

For weight $2-k=\frac{1}{2}$, we have a conditional result. To make it precise, we say that a form $f \in H_{\frac{1}{2}}(N, \chi)$ is good if the Maass-Poincaré series corresponding to nontrivial terms in the principal parts of $f$ are individually convergent.

THeOREM 11.3. If $f \in H_{\frac{1}{2}}(N, \chi)$ is good, then there is a finite set $S_{\Theta}(f)$ of complex numbers such that for positive $n$ we have

$$
c_{f}^{+}(n)=\mathcal{C}(f, \infty ; n)+\mu
$$

for some $\mu \in S_{\Theta}(f)$. Moreover, if $n \neq d m^{2}$ for some $d \mid N$ and $m \in \mathbb{Z}^{+}$, then $\mu=0$.

REMARK 38. We believe that all $f \in H_{\frac{1}{2}}(N, \chi)$ are good. In earlier work we deduced convergence of such Maass-Poincaré series by making using of relationships between Kloosterman sums and Salié sums (see Section 4 of [53]), and by generalizing work of Goldfeld and Sarnak [100] on sums of Kloosterman sums (see $[\mathbf{9 6}]$ ). It seems likely that a careful application of these ideas will prove that each such $f$ is indeed good.

Sketch of the Proof of Theorem 11.2 And 11.3. By the general theory of Maass-Poincaré series, which generalizes Section 8.3, we have an explicit linear combination of Maass-Poincaré series, say $\mathfrak{f} \in H_{2-k}(N, \chi)$, whose principal parts agree with the principal parts of $f$ up to additive constants. The complex number $\mathcal{C}(f, \infty ; n)$ is the $n$th coefficient of the holomorphic part of this linear combination. For the weight $2-k=1 / 2$, one must argue further (for example, using the equidistribution of $\mathrm{CM}$ points) to establish that these formulas are convergent.

There are then three possibilites:

Case 1. We have that $f-\mathfrak{f}$ is a holomorphic modular form. It can only be nonzero when $2-k=\frac{1}{2}$, in which case the Serre-Stark Basis Theorem [189] implies that $f-\mathfrak{f}$ is a linear combination of theta functions, giving Theorem 11.3.

Case 2. We have that $f-\mathfrak{f}$ is a weakly holomorphic modular form which is not a holomorphic modular form. Such a form must have a pole at a cusp. However, this cannot happen since we constructed $\mathfrak{f}$ so that the principal parts of $f-\mathfrak{f}$ are constant.

Case 3. We have that $f-\mathfrak{f}$ is a harmonic Maass form with a non-trivial nonholomorphic part. However, Lemma 7.5 shows that all such harmonic Maass 
forms have at least one principal part which is nonconstant. Therefore, this possibility never occurs.

\section{Applications to modular forms}

Here we use the theory of harmonic Maass forms to address two classical problems in the theory of modular forms:

- Linear relations among Poincaré series

- Vanishing of Hecke eigenvalues.

We are able to obtain new results by making use of Lemma 7.4, the fact that

$$
\xi_{2-k}: H_{2-k}(\Gamma) \longrightarrow S_{k}(\Gamma) .
$$

These classical problems, which pertain to properties of the Fourier coefficients of cusp forms in $S_{k}(\Gamma)$, can be investigated by leveraging information from harmonic Maass forms in $H_{2-k}(\Gamma)$.

12.1. Relations among classical Poincaré series. It is well known that the Poincaré series (see Section 8.3)

$$
\{P(m, k, N ; z): m \geq 1\}
$$

span the space $S_{k}(N)$. Since the space $S_{k}(N)$ is finite dimensional, there exist many relations among these Poincaré series. In his classic text on automorphic forms, Iwaniec [125] states the following seemingly simple problem.

Problem. Find all the linear relations between $P(m, k, N ; z)$.

REMARK 39. Iwaniec states this problem for more general multiplier systems, but the techniques here also work in that setting.

The next theorem, due to Rhoades (see $[\mathbf{1 7 8}, \mathbf{1 7 9}]$ ), follows from the theory of harmonic Maass forms.

TheOREM 12.1. Suppose that $2 \leq k \in \frac{1}{2} \mathbb{Z}$, and suppose that $\mathcal{I}$ is a finite set of positive integers. Then we have that

$$
\sum_{m \in \mathcal{I}} \alpha_{m} P(m, k, N ; z) \equiv 0
$$

if and only if there is a form in $M_{2-k}^{!}(N)$ whose principal part at $\infty$ is

$$
\sum_{m \in \mathcal{I}} \frac{\alpha_{m}}{m^{k-1}} \cdot q^{-m}
$$

with trivial principal parts at all other cusps.

EXAMPLE 12.2. In practice it is not difficult to implement Theorem 12.1. For example, consider the two dimensional space $S_{24}(1)$. Obviously, there is 
a linear relation between the Poincaré series $P(1,24,1 ; z), P(2,24,1 ; z)$ and $P(3,24,1 ; z)$. Using the formulas in Theorem 8.3 , we find that

$$
\begin{aligned}
& P(1,24,1 ; z) \sim 1.00100852 \cdot q+132.988977 \cdot q^{2}+189296.261 \cdot q^{3}+\cdots \\
& P(2,24,1 ; z) \sim 0.00001585 \cdot q+2.45743136 \cdot q^{2}+114.854805 \cdot q^{3}+\cdots \\
& P(3,24,1 ; z) \sim 0.00000201 \cdot q+0.01023411 \cdot q^{2}+0.88465633 \cdot q^{3}+\cdots .
\end{aligned}
$$

From these numerics, we find that

$-0.00000207832 \cdot P(1,24,1 ; z)+0.00427703 \cdot P(2,24,1 ; z)+P(3,24,1 ; z) \sim 0$.

Although it is not possible to make this approximation precise by using explicit Fourier expansions, Theorem 12.1 reduces this problem to a simple calculation. We find the exact relation thanks to the existence of the modular form $E_{14} / \Delta^{3}$

$$
\frac{E_{14}(z)}{\Delta(z)^{3}}=\frac{1}{q^{3}}+\frac{48}{q^{2}}-\frac{195660}{q}+\cdots .
$$

Therefore, we then find that the two coefficients in the linear combination above are exactly

$$
\frac{-195660}{3^{23}} \approx-0.00000207832 \ldots \text { and } \frac{48 \cdot 2^{23}}{3^{23}} \approx 0.00427703 \ldots
$$

Theorem 12.1 is a simple consequence of the theory of harmonic Maass forms.

Proof of Theorem 12.1. Assume that $\sum_{m \in \mathcal{I}} \alpha_{m} P(m, k, N ; z) \equiv 0$. Define a weight $2-k$ harmonic Maass form $f$ by

$$
\begin{aligned}
f & =\sum_{m \in \mathcal{I}} \frac{\alpha_{m}}{m^{k-1}} \cdot Q(-m, k, N ; z) \\
& =\sum_{n \gg-\infty} c_{f}^{+}(n) q^{n}+\sum_{n<0} c_{f}^{-}(n) \Gamma(k-1,4 \pi|n| y) q^{n} .
\end{aligned}
$$

By Lemma 7.4 and Theorem 8.5, we find that

$$
\begin{aligned}
\xi_{2-k}(f) & =(4 \pi)^{k-1}(k-1) \sum_{m \in \mathcal{I}} \alpha_{m} P(m, k, N ; z) \\
& =-(4 \pi)^{k-1}(k-1) \sum_{n \geq 1} \overline{c_{f}^{-}(-n)} n^{k-1} q^{n} .
\end{aligned}
$$

By our assumption on the sum over the Poincaré series, we know that $c_{f}^{-}(n)=0$ for all $n<0$, and so $f \in M_{2-k}^{!}(N)$. Namely, in terms of the Maass-Poincaré series $Q(-m, k, N ; z)$ in Section 8.3, we have that

$$
\sum_{m \in I} \frac{\alpha_{m}}{m^{k-1}} \cdot Q^{+}(-m, k, N ; z) \in M_{2-k}^{!}(N)
$$

is the weakly holomorphic form that we desire. 
Conversely, assume that $f$ is such a weakly holomorphic modular form. From the expansion for the coefficients of $Q^{+}(-m, k, N ; z)$ in Theorem 8.4, we find that

$$
\tilde{f}:=-f+\frac{1}{\Gamma(k)} \cdot \sum_{m \in \mathcal{I}} \frac{\alpha_{m}}{m^{k-1}} \cdot Q(-m, k, N ; z) \in H_{2-k}(N)
$$

is a harmonic Maass form with trivial principal parts at every cusp. By Lemma 7.5 , it then follows that $\tilde{f}$ is in $M_{2-k}^{!}(N)$. Theorem 8.5 then implies that $0=\xi_{2-k}(\widetilde{f})=\sum_{m \in \mathcal{I}} \alpha_{m} P(m, k, N ; z)$.

12.2. Algebraicity and the vanishing of Hecke eigenvalues. Despite the fact that we have a fairly complete theory of algebraicity for forms in $M_{k}^{!}(N, \chi)$, thanks to the $q$-expansion principle, the theory of Eisenstein series and newforms, the question of algebraicity for harmonic Maass forms remains open. As we shall now see, these questions (in the case of integer weight) are intimately related to the vanishing of eigenvalues of Hecke operators.

REMARK 40. The algebraicity of the coefficients of half-integral weight harmonic Maass forms is much more mysterious. For certain weight $1 / 2$ forms, we shall relate algebraicity to the vanishing of derivatives of quadratic twists of weight 2 modular $L$-functions (see Section 15 ).

To make this precise, we shall restrict our attention to those $f \in H_{2-k}$ $(N, \chi)$ for which $\xi_{2-k}(f) \in S_{k}(N, \bar{\chi})$ is a Hecke eigenform. For reasons which will become apparent, we shall concentrate on those forms for which

$$
\xi_{2-k}(f)=\frac{g}{\|g\|^{2}},
$$

where $g$ is a normalized newform and $\|g\|$ denotes its usual Petersson norm.

To illustrate the nature of our result, we consider two examples of MaassPoincaré series which are not weakly holomorphic modular forms. The Maass-Poincaré series $f:=\frac{1}{11 !} \cdot Q(-1,12,1 ; z) \in H_{-10}(1)$ satisfies (12.1) for $g=\Delta(z)$, the unique normalized weight 12 cusp form on the full modular group. The first few coefficients ${ }^{17}$ of its holomorphic part are

$$
\frac{1}{11 !} \cdot Q^{+}(-1,12,1 ; z) \sim q^{-1}-\frac{65520}{691}-1842.89472 q-23274.07545 q^{2}-\cdots \text {. }
$$

There is little reason to believe that these coefficients are rational or algebraic. On the other hand, we shall prove that the Maass-Poincaré series $\frac{1}{3 !} \cdot Q(-1,4,9 ; z) \in H_{-2}(9)$ has the property that $\frac{1}{3 !} \cdot Q^{+}(-1,4,9 ; z)$ has rational coefficients. Its first few terms are

$$
\frac{1}{3 !} \cdot Q^{+}(-1,4,9 ; z)=q^{-1}-\frac{1}{4} q^{2}+\frac{49}{125} q^{5}-\frac{48}{512} q^{8}-\frac{771}{1331} q^{11}+\cdots
$$

and $f:=\frac{1}{3 !} \cdot Q(-1,4,9 ; z)$ satisfies $(12.1)$ for the unique normalized newform in $S_{4}(9)$.

\footnotetext{
${ }^{17}$ This corrects a typographical error for the constant term in [68].
} 
Our next theorem explains the distinction between these two cases. To make this precise, let $g \in S_{k}(N, \bar{\chi})$ be a normalized newform, and let $F_{g}$ be the number field obtained by adjoining the coefficients of $g$ to $\mathbb{Q}$.

DeFinition 12.3. Assuming the hypotheses above, we say that a harmonic Maass form $f \in H_{2-k}(N, \chi)$ is good for $g$ if it satisfies the following properties:

(i) The principal part of $f$ at the cusp $\infty$ belongs to $F_{g}\left[q^{-1}\right]$.

(ii) The principal parts of $f$ at the other cusps of $\Gamma_{0}(N)$ are constant.

(iii) We have that $\xi_{2-k}(f)=\|g\|^{-2} g$.

REMARK 41. For every such $g$, there is an $f$ which is good for $g$. Moreover, such an $f$ is unique up to a weakly holomorphic form in $M_{2-k}^{!}(N, \chi)$ with coefficients in $F_{g}$. Such $f$ can be constructed explicitly using Poincaré series.

In joint work with Bruinier and Rhoades [68], the author has obtained the following result which explains this phenomenon.

Theorem 12.4. Let $g \in S_{k}(N, \bar{\chi})$ be a normalized newform with complex multiplication (see (12.6)). If $f \in H_{2-k}(N, \chi)$ is good for $g$, then all coefficients of $f^{+}$are in $F_{g}\left(\zeta_{M}\right)$, where $\zeta_{M}:=e^{2 \pi i / M}$ and $M=N D$, where $D$ is the discriminant of the field of complex multiplication.

REMARK 42.

i) The rationality of $Q^{+}(-1,4,9 ; z)$ in $(12.2)$ is an example of Theorem 12.4. In this case $Q(-1,4,9 ; z)$ is good for $\eta(3 z)^{8}$, the unique CM newform in $S_{4}(9)$.

ii) The field $F_{g}$ in Theorem 12.4 is explicit, and is determined by Hecke characters.

iii) Let $g \in S_{k}(N, \bar{\chi})$ and $f \in H_{2-k}(N, \chi)$ be as in Theorem 12.4. The proof of the theorem implies that all of the coefficients of $f^{+}$belong to $F_{g}\left(c_{f}^{+}(1)\right)$. In fact, the proof gives this conclusion for any newform $g$, not just those with CM.

iv) In the examples we know, it turns out that the coefficients of $f^{+}$are actually contained in $F_{g}$. It is natural to ask whether this is true in general.

The proof of Theorem 12.4 relies on the fact that some Hecke eigenvalues of $g$ vanish. A simple generalization of the proof of Theorem 12.4 can be used to detect the vanishing of the Fourier coefficients of a newform.

THEOREM 12.5. Suppose that $g=\sum_{n=1}^{\infty} c_{g}(n) q^{n} \in S_{k}(N, \bar{\chi})$ is a normalized newform, and suppose that $f \in H_{2-k}(N, \chi)$ is good for $g$. If $p \nmid N$ is a prime for which $c_{g}(p)=0$, then $c_{f}^{+}(p n)$ is algebraic for every positive $n$ coprime to $p$.

As in Remark 42 iv), it seems possible that the algebraic coefficients of $f^{+}$are always in $F_{g}$. We address this in the next example when $N=1$. 
EXAMPLE 12.6. Here we consider Lehmer's Conjecture on the nonvanishing of Ramanujan's $\tau$-function, where

$$
\Delta(z)=\sum_{n=1}^{\infty} \tau(n) q^{n}
$$

This example generalizes easily to all level 1 Hecke eigenforms.

Although Theorem 12.5 relates Lehmer's Conjecture to the alleged transcendence of the coefficients, say $a_{\Delta}(n)$, of $\frac{1}{11 !} \cdot Q^{+}(-1,12,1 ; z)$, it turns out that more is true. Lehmer's Conjecture is implied by the irrationality of these coefficients.

We make use of explicit formulas. Using the classical Eisenstein series $E_{4}$ and $E_{6}$, and the classical $j$-function $j(z)$, we define polynomials $J_{m}(x) \in \mathbb{Z}[x]$ by

$$
\sum_{m=0}^{\infty} J_{m}(x) q^{m}:=\frac{E_{4}(z)^{2} E_{6}(z)}{\Delta(z)} \cdot \frac{1}{j(z)-x}=1+(x-744) q+\cdots .
$$

For each $m$ we then let $j_{m}(z)=J_{m}(j(z))$. If $p$ is prime, then define the modular functions

$$
\begin{gathered}
A_{p}(z):=\frac{24}{B_{12}}\left(1+p^{11}\right)+j_{p}(z)-264 \sum_{m=1}^{p} \sigma_{9}(m) j_{p-m}(z), \\
B_{p}(z):=-\tau(p)\left(-264+\frac{24}{B_{12}}+j_{1}(z)\right) .
\end{gathered}
$$

Here $B_{12}=-\frac{691}{2730}$ is the 12 th Bernoulli number. Using the principal part of $Q(-1,12,1 ; z)$ combined with the fact that $\Delta(z)$ is an eigenform of the Hecke algebra, Theorem 7.10 implies (also see [166]), for primes $p$, that

$$
\sum_{n=-p}^{\infty}\left(p^{11} a_{\Delta}(p n)-\tau(p) a_{\Delta}(n)+a_{\Delta}(n / p)\right) q^{n}=\frac{A_{p}(z)+B_{p}(z)}{E_{4}(z) E_{6}(z)} .
$$

These weight -10 modular forms have integer coefficients. Now suppose that $\tau(p)=0$ for a prime $p$. Then $a_{\Delta}(n p)$ is rational for every $n$ coprime to $p$.

REMARK 43. Results similar to the example above have also been obtained recently by Boylan and Guerzhoy $[45,109]$.

Here we prove Theorem 12.4 by combining facts about $\xi_{2-k}$, with Hecke theory and the theory of complex multiplication. We first begin with an important proposition.

Proposition 12.7. Let $g=\sum_{n=1}^{\infty} b(n) q^{n} \in S_{k}(N, \bar{\chi})$ be a normalized newform with integer weight $k \geq 2$, and let $F_{g}$ be the number field obtained by adjoining the coefficients of $g$ to $\mathbb{Q}$. Then there is a harmonic Maass form $f \in H_{2-k}(N, \chi)$ which satisfies:

(i) The principal part of $f$ at the cusp $\infty$ belongs to $F_{g}\left[q^{-1}\right]$. 
(ii) The principal parts of $f$ at the other cusps of $\Gamma_{0}(N)$ are constant.

(iii) We have that $\xi_{2-k}(f)=\|g\|^{-2} g$.

Proof. Let $H_{2-k, \infty}(N, \chi)$ be the subspace of those $f \in H_{2-k}(N, \chi)$ whose principal parts at the cusps other than $\infty$ are constant. Note that

$$
H_{2-k}(N, \chi)=H_{2-k, \infty}(N, \chi)+M_{2-k}^{!}(N, \chi) .
$$

Arguing as in Section 3 of [63], the restriction of $\xi_{2-k}$ to $H_{2-k, \infty}(N, \chi)$ defines a surjective map to $S_{k}(N, \bar{\chi})$. One now argues as in the proof of Lemma 7.3 of [67] using the pairing $\{g, f\}=\left(g, \xi_{2-k}(f)\right)$, where $f \in H_{2-k, \infty}(N, \chi)$ and $g \in S_{k}(N, \bar{\chi})$.

REMARK 44. The harmonic Maass form $f$ satisfying (i)-(iii) is unique up to the addition of a weakly holomorphic form in $M_{2-k}^{!}(N, \chi)$ with coefficients in $F_{g}$ and a pole possibly at infinity and constant principal part at all other cusps.

For completeness, here we briefly recall the notion of a newform with complex multiplication (for example, see Chapter 12 of [125] or Section 1.2 of [164]). Let $D<0$ be the fundamental discriminant of an imaginary quadratic field $K=\mathbb{Q}(\sqrt{D})$. Let $O_{K}$ be the ring of integers of $K$, and let $\chi_{K}:=\left(\frac{D}{\bullet}\right)$ be the usual Kronecker character associated to $K$. Let $k \geq 2$, and let $c$ be a Hecke character of $K$ with exponent $k-1$ and conductor $\mathfrak{f}_{c}$, a non-zero ideal of $O_{K}$. By definition, this means that

$$
c: I\left(\mathfrak{f}_{c}\right) \longrightarrow \mathbb{C}^{\times}
$$

is a homomorphism, where $I\left(\mathfrak{f}_{c}\right)$ denotes the group of fractional ideals of $K$ prime to $\mathfrak{f}_{c}$, and

$$
c\left(\alpha O_{K}\right)=\alpha^{k-1}
$$

for $\alpha \in K^{\times}$for which $\alpha \equiv 1 \bmod ^{\times} \mathfrak{f}_{c}$. To $c$ we naturally associate a Dirichlet character $\omega_{c}$ defined, for every integer $n$ coprime to $\mathfrak{f}_{c}$, by

$$
\omega_{c}(n):=\frac{c\left(n O_{K}\right)}{n^{k-1}} .
$$

We then let

$$
\Phi_{K, c}(z):=\sum_{\mathfrak{a}} c(\mathfrak{a}) q^{N(a)},
$$

where $\mathfrak{a}$ varies over the ideals of $O_{K}$ prime to $\mathfrak{f}_{c}$, and where $N(\mathfrak{a})$ is the usual ideal norm. It is well known that $\Phi_{K, c}(z) \in S_{k}\left(|D| \cdot N\left(\mathfrak{f}_{c}\right), \chi_{K} \cdot \omega_{c}\right)$ is a normalized newform. These are newforms with complex multiplication. By construction, if we let

$$
\Phi_{K, c}(z)=\sum_{n=1}^{\infty} b(n) q^{n},
$$

then

$$
b(n)=0 \quad \text { whenever } \chi_{K}(n)=-1 .
$$


This follows since every prime $p$ for which $\chi_{K}(p)=-1$ is inert.

Proof of Theorem 12.4. Suppose that $f$ is good for a CM form $g=$ $\sum_{n=1}^{\infty} b(n) q^{n}$, and let $D=D_{g}$ be the fundamental discriminant of the associated imaginary quadratic field $K=\mathbb{Q}(\sqrt{D})$. By Lemma 7.4, we then have that

$$
\xi_{2-k}(f)=\|g\|^{-2} g=-(4 \pi)^{k-1} \sum_{n=1}^{\infty} \overline{c_{f}^{-}(-n)} n^{k-1} q^{n} .
$$

Since $g$ has complex multiplication, (12.7) implies that $c_{f}^{-}(n)=0$ when $\chi_{K}(-n)=-1$. Because $D<0$, this means that

$$
c_{f}^{-}(n)=0 \text { when } \chi_{K}(n)=1 .
$$

Let $M=N D$. We write $\chi_{0}$ for the trivial character modulo $|D|$. Since $D \mid N$, a simple generalization of standard facts about twists of modular forms implies that

$$
u:=f \otimes \chi_{0}+f \otimes \chi_{K}
$$

is in $H_{2-k}(M, \chi)$. The Fourier expansion of $u=u^{+}+u^{-}$is given by

$$
\begin{aligned}
& u^{+}(z)=2 \sum_{\substack{n \gg-\infty \\
\chi_{K}(n)=1}} c_{f}^{+}(n) q^{n}, \\
& u^{-}(z)=2 \sum_{\substack{n<0 \\
\chi_{K}(n)=1}} c_{f}^{-}(n) \Gamma(k-1,4 \pi|n| y) q^{n} .
\end{aligned}
$$

The non-holomorphic part $u^{-}$vanishes, and so $u$ is a weakly holomorphic modular form.

We now claim that for any integer $b, f(z+b / D)$ has principal parts at all cusps in $F_{g}\left(\zeta_{M}\right)\left[q^{-1}\right]$. To see this, we let $\gamma \in \Gamma(1)$ and consider the cusp $\gamma \infty$. There exists a $\widetilde{\gamma} \in \Gamma(1)$ and $\alpha, \beta, \delta \in \mathbb{Z}$ such that

$$
\left(\begin{array}{cc}
D & b \\
0 & D
\end{array}\right) \gamma=\widetilde{\gamma}\left(\begin{array}{cc}
\alpha & \beta \\
0 & \delta
\end{array}\right)
$$

Hence, the Fourier expansion of $f(z+b / D)$ at the cusp $\gamma \infty$ is given by

$$
f|\widetilde{\gamma}|\left(\begin{array}{cc}
\alpha & \beta \\
0 & \delta
\end{array}\right) \text {. }
$$

By the assumption of $f$, it is holomorphic at the $\operatorname{cusp} \infty$, unless $\widetilde{\gamma} \in \Gamma_{0}(N)$, in which case it is equal to

$$
f \mid\left(\begin{array}{cc}
\alpha & \beta \\
0 & \delta
\end{array}\right)
$$

Since $\delta\left|D^{2}\right| M$, the principal part at $\infty$ of this modular form is contained in $F_{g}\left(\zeta_{M}\right)\left[q^{-1}\right]$, proving the claim. This implies that the twists $f \otimes \chi_{0}$, $f \otimes \chi_{D}$, have principal parts at all cusps in $F_{g}\left(\zeta_{M}\right)\left[q^{-1}\right]$. Therefore, the same is true for $u$. 
The action of $\operatorname{Aut}\left(\mathbb{C} / \mathbb{Q}\left(\zeta_{N}\right)\right)$ commutes with the action of $S L_{2}(\mathbb{Z})$ on modular functions for $\Gamma(N)$ (for example, see Theorem 6.6 in Chapter 6.2 and the diagram before Remark 6.7 in Shimura's book [193]). Using the action of $\operatorname{Aut}\left(\mathbb{C} / F_{g}\left(\zeta_{M}\right)\right)$ on weakly holomorphic modular forms, we see that $u^{\sigma}$ has the same properties for any $\sigma \in \operatorname{Aut}\left(\mathbb{C} / F_{g}\left(\zeta_{M}\right)\right)$. Moreover, $u^{\sigma}$ has the same principal parts as $u$ at all cusps. Hence the difference $u-u^{\sigma}$ is a holomorphic modular form which vanishes at the cusp $\infty$. Since $2-k \leq 0$, this implies that $u=u^{\sigma}$. Consequently, $u$ is defined over $F_{g}\left(\zeta_{M}\right)$. So for all $n \in \mathbb{Z}$ with $\chi_{K}(n)=1$, we have that $c_{f}^{+}(n) \in F_{g}\left(\zeta_{M}\right)$. In particular, $c_{f}^{+}(1) \in F_{g}\left(\zeta_{M}\right)$.

We now use the Hecke action on $f$ and $g$. By Theorem 7.10, we have that

$$
\left.f\right|_{2-k} T(m)=m^{1-k} b(m) f+f^{\prime},
$$

where $f^{\prime} \in M_{2-k}^{!}\left(\Gamma_{0}(N), \chi\right)$ is a weakly holomorphic form with coefficients in $F_{g}$. In view of the formula for the action of the Hecke operators on the Fourier expansion, we obtain for any prime $p$ that

$$
c_{f}^{+}(p n)+\chi(p) p^{1-k} c_{f}^{+}(n / p)=p^{1-k} b(p) c_{f}^{+}(n)+c_{f^{\prime}}^{+}(n),
$$

where $c_{f^{\prime}}^{+}(n) \in F_{g}$. Hence an inductive argument shows that all coefficients $c_{f}^{+}(n)$ are contained in the extension $F_{g}\left(c_{f}^{+}(1)\right)$. This concludes the proof of the theorem since we have already established that $c_{f}^{+}(1)$ is in $F_{g}\left(\zeta_{M}\right)$.

The proof of Theorem 12.5 is similar to the proof of Theorem 12.4, and so we only give a brief indication of how the proof must be modified.

Sketch Proof of Theorem 12.5. If $p \nmid N$ is a prime, then for every positive integer $m$ we have that

$$
c_{g}(p) c_{g}\left(p^{m}\right)=c_{g}\left(p^{m+1}\right)+\overline{\chi(p)} p^{k-1} c_{g}\left(p^{m-1}\right) .
$$

Therefore, if $p \nmid N$ is a prime for which $c_{g}(p)=0$, then we have that

$$
c_{g}\left(p^{m+1}\right)=-\overline{\chi(p)} p^{k-1} c_{g}\left(p^{m-1}\right),
$$

which in turn implies that

$$
c_{g}\left(p^{m}\right)= \begin{cases}\left(-\overline{\chi(p)} p^{k-1}\right)^{\frac{m}{2}} & \text { if } m \text { is even } \\ 0 & \text { otherwise. }\end{cases}
$$

A standard argument gives a harmonic Maass form whose Fourier coefficients are supported on terms whose exponents $n$ have the property that $p$ exactly divides $n$. By the multiplicativity of the Fourier coefficients of newforms, it then follows by the observation above that the non-holomorphic part of this form is identically zero. In other words, this particular harmonic Maass form is a weakly holomorphic modular form with suitable principal parts at cusps. The proof now follows mutatis mutandis as in the proof of Theorem 12.4. 


\section{Generating functions for singular moduli}

Let $j(z)$ be the usual modular function for $\mathrm{SL}_{2}(\mathbb{Z})$

$$
j(z)=q^{-1}+744+196884 q+21493760 q^{2}+\cdots .
$$

The values of modular functions such as $j(z)$ at imaginary quadratic arguments in $\mathbb{H}$ are known as singular moduli. Singular moduli are algebraic integers which play many roles in number theory. For example, they generate class fields of imaginary quadratic fields, and they parameterize isomorphism classes of elliptic curves with complex multiplication.

In an important paper, Zagier $[\mathbf{2 1 2}]$ proved that the traces of these numbers are Fourier coefficients of certain weight $3 / 2$ modular forms. To illustrate his results, we begin by comparing the classical evaluations

$$
\begin{gathered}
\frac{j\left(\frac{-1+\sqrt{-3}}{2}\right)-744}{3}=-248, \quad \frac{j(i)-744}{2}=492, \\
j\left(\frac{1+\sqrt{-7}}{2}\right)-744=-4119
\end{gathered}
$$

with the Fourier coefficients of the modular form

$$
\begin{aligned}
g(z):=-\frac{\eta(z)^{2} \cdot E_{4}(4 z)}{\eta(2 z) \eta(4 z)^{6}}= & -q^{-1}+2-248 q^{3} \\
& +492 q^{4}-4119 q^{7}+7256 q^{8}-\cdots
\end{aligned}
$$

The appearance of singular moduli as the initial coefficients of the modular form $g(z)$ is not a coincidence.

We now make this more precise. For integers $\lambda$, let $\mathcal{M}_{\lambda+\frac{1}{2}}^{!}(4)$ be the space of weight $\lambda+\frac{1}{2}$ weakly holomorphic modular forms on $\Gamma_{0}(4)$ satisfying the "Kohnen plus-space" condition. A form satisfies this condition if its $q$ expansion has the form

$$
\sum_{(-1)^{\lambda} n \equiv 0,1} a(n) q^{n}
$$

Throughout let $d \equiv 0,3(\bmod 4)$ be a positive integer, let $H(d)$ be the Hurwitz-Kronecker class number for the discriminant $-d$, and let $\mathcal{Q}_{d}$ be the set of positive definite integral binary quadratic forms (note. including imprimitive forms)

$$
Q(x, y)=[a, b, c]=a x^{2}+b x y+c y^{2}
$$

with discriminant $D_{Q}=-d=b^{2}-4 a c$. For each $Q$, let $\tau_{Q}$ be the unique root in $\mathbb{H}$ of $Q(x, 1)=0$. The singular modulus $f\left(\tau_{Q}\right)$, for any modular invariant $f(z)$, depends only on the equivalence class of $Q$ under the action 
of $\Gamma:=\mathrm{PSL}_{2}(\mathbb{Z})$. If $\omega_{Q} \in\{1,2,3\}$ is given by

$$
\omega_{Q}:= \begin{cases}2 & \text { if } Q \sim_{\Gamma}[a, 0, a], \\ 3 & \text { if } Q \sim_{\Gamma}[a, a, a], \\ 1 & \text { otherwise }\end{cases}
$$

then, for a modular invariant $f(z)$, define the trace $\operatorname{Tr}(f ; d)$ by

$$
\operatorname{Tr}(f ; d):=\sum_{Q \in \mathcal{Q}_{d} / \Gamma} \frac{f\left(\tau_{Q}\right)}{\omega_{Q}} .
$$

Theorems 1 and 5 of Zagier's paper [212] imply the following theorem.

THEOREM 13.1. If $f(z) \in \mathbb{Z}[j(z)]$ has a Fourier expansion with constant term 0 , then there is a finite principal part $A_{f}(z)=\sum_{n \leq 0} a_{f}(n) q^{n}$ for which

$$
A_{f}(z)+\sum_{0<d \equiv 0,3(\bmod 4)} \operatorname{Tr}(f ; d) q^{d} \in \mathcal{M}_{\frac{3}{2}}^{!}(4) .
$$

REMARK 45. The earlier claim in (13.1) is the $f(z)=j(z)-744$ case of this theorem.

REMARK 46. Zagier's paper [212] includes many generalizations of this theorem. One of his results proves that the generating functions of the traces of $j_{m}(z):=m(j(z)-744) \mid T(m)$ are also explicit weight $3 / 2$ weakly holomorphic modular forms. This result is of particular significance because it reduces the computation of Hilbert class polynomials to a straightforward calculation.

13.1. Further generating functions for traces of singular moduli. Zagier's work has inspired an enormous number of works $[\mathbf{5 4}, \mathbf{5 8}, \mathbf{6 4}$, $65,77,78,87,88,89,95,127,128,154]$ by the author, Bringmann, Bruinier, D. Choi, W. Duke, A. Folsom, J. Funke, O. Imamoḡlu, P. Jenkins, D. Jeon, S.-Y. Kang, C. Kim, R. Masri, A. Miller, A. Pixton, J. Rouse, and A. Toth. These papers give theorems related to the phenomenon that the coefficients of modular forms and harmonic Maass forms are often the "traces" of some sort of singular moduli. For brevity, here we discuss one construction which is based on Maass-Poincaré series.

For fundamental discriminants $D_{1}$, let $\chi_{D_{1}}$ denote the associated genus character for positive definite binary quadratic forms whose discriminants are multiples of $D_{1}$. If $\lambda$ is an integer and $D_{2}$ is a non-zero integer for which $(-1)^{\lambda} D_{2} \equiv 0,1(\bmod 4)$ and $(-1)^{\lambda} D_{1} D_{2}<0$, then define the twisted trace of a modular invariant $f(z)$, say $\operatorname{Tr}_{D_{1}}\left(f ; D_{2}\right)$, by

$$
\operatorname{Tr}_{D_{1}}\left(f ; D_{2}\right):=\sum_{Q \in \mathcal{Q}_{\left|D_{1} D_{2}\right|} / \Gamma} \frac{\chi_{D_{1}}(Q) f\left(\tau_{Q}\right)}{\omega_{Q}} .
$$

Many of the works mentioned above prove that such traces are often coefficients of Maass forms and weakly holomorphic modular forms. Here we 
give one illustrative example of this phenomenon using some of the Poincaré series constructed in Section 8.3. For convenience, we briefly recall their construction. Let $k:=\lambda+\frac{1}{2}$, where $\lambda$ is an arbitrary integer, and let $M_{\nu, \mu}(z)$ be the usual $M$-Whittaker function. For $s \in \mathbb{C}$ and $y \in \mathbb{R}-\{0\}$, we define

$$
\mathcal{M}_{s}(y):=|y|^{-\frac{k}{2}} M_{\frac{k}{2} \operatorname{sgn}(y), s-\frac{1}{2}}(|y|) .
$$

Suppose that $m \geq 1$ is an integer with $(-1)^{\lambda+1} m \equiv 0,1(\bmod 4)$. As before, let

$$
\varphi_{-m, s}(z):=\mathcal{M}_{s}(-4 \pi m y) e(-m x)
$$

where $z=x+i y$ and $e(w):=e^{2 \pi i w}$, and let $\Gamma_{\infty}$ denote the translations in $\mathrm{SL}_{2}(\mathbb{Z})$. With this notation, define the Poincaré series

$$
\mathcal{F}_{\lambda}(-m, s ; z):=\sum_{A \in \Gamma_{\infty} \backslash \Gamma_{0}(4)}\left(\left.\varphi_{-m, s}\right|_{k} A\right)(z)
$$

for $\operatorname{Re}(s)>1$. Here $\left.\right|_{k}$ denotes the usual half-integral weight $k$ "slash operator". If $\mathrm{pr}_{\lambda}$ is Kohnen's projection operator (see page 250 of [136]) to the weight $\lambda+\frac{1}{2}$ plus-space for $\Gamma_{0}(4)$, then for $\lambda \notin\{0,1\}$ define $F_{\lambda}(-m ; z)$ by

$$
F_{\lambda}(-m ; z):= \begin{cases}\frac{3}{2} \mathcal{F}_{\lambda}\left(-m, \frac{k}{2} ; z\right) \mid \operatorname{pr}_{\lambda} & \text { if } \lambda \geq 2 \\ \frac{3}{2(1-k) \Gamma(1-k)} \mathcal{F}_{\lambda}\left(-m, 1-\frac{k}{2} ; z\right) \mid \operatorname{pr}_{\lambda} & \text { if } \lambda \leq-1\end{cases}
$$

REMARK 47. For $\lambda=0$ or 1 we also have series $F_{\lambda}(-m ; z)$. Their construction requires more care due to questions of convergence (see [54, $65])$.

Arguing as in Section 8.3, if $\lambda \geq-6$ with $\lambda \neq-5$, then $F_{\lambda}(-m ; z) \in$ $\mathcal{M}_{\lambda+\frac{1}{2}}^{!}(4)$. For such $\lambda$, we denote the corresponding Fourier expansions by

$$
F_{\lambda}(-m ; z)=q^{-m}+\sum_{\substack{n \geq 0 \\(-1)^{\lambda} n \equiv 0,1}} b_{\lambda}(-m ; n) q^{n} \in \mathcal{M}_{\lambda+\frac{1}{2}}^{!}(4) .
$$

For other $\lambda$, namely $\lambda=-5$ or $\lambda \leq-7$, it turns out that the $F_{\lambda}(-m ; z)$ are Maass forms in $H_{\lambda+\frac{1}{2}}(4)$. We denote their expansions by

$$
F_{\lambda}(-m ; z)=B_{\lambda}(-m ; z)+q^{-m}+\sum_{\substack{n \geq 0 \\(-1)^{\lambda} n \equiv 0,1(\bmod 4)}} b_{\lambda}(-m ; n) q^{n},
$$

where $B_{\lambda}(-m ; z)$ is the "non-holomorphic" part of $F_{\lambda}(-m ; z)$.

ExAMPLE 13.2. If $\lambda=1$ and $-m=-1$, then we find the form in (13.1)

$$
-F_{1}(-1 ; z)=g(z)=-q^{-1}+2-248 q^{3}+492 q^{4}-4119 q^{7}+7256 q^{8}-\cdots .
$$


Generalizing Zagier's results, we have that the coefficients $b_{\lambda}(-m ; n)$ of the $F_{\lambda}(-m ; z)$ are traces of singular moduli for functions defined by Niebur [160]. If $I_{s}(x)$ denotes the usual $I$-Bessel function, and if $\lambda>1$, then let

$$
\mathfrak{F}_{\lambda}(z):=\pi \sum_{A \in \Gamma_{\infty} \backslash \mathrm{SL}_{2}(\mathbb{Z})} \operatorname{Im}(A z)^{\frac{1}{2}} I_{\lambda-\frac{1}{2}}(2 \pi \operatorname{Im}(A z)) e(-\operatorname{Re}(A z)) .
$$

For $\lambda=1$, a similar construction holds. Indeed, Niebur [160] showed that $\mathfrak{F}_{1}(z)=\frac{1}{2}(j(z)-744)$, where this function is the analytic continuation, as $s \rightarrow 1^{+}$, of

$$
-12+\pi \sum_{A \in \Gamma_{\infty} \backslash \mathrm{SL}_{2}(\mathbb{Z})} \operatorname{Im}(A z)^{\frac{1}{2}} I_{s-\frac{1}{2}}(2 \pi \operatorname{Im}(A z)) e(-\operatorname{Re}(A z)) .
$$

We then have the following theorem.

Theorem 13.3. (Bringmann and Ono; Theorem 1.2 of [54]) If $\lambda, m \geq 1$ are integers for which $(-1)^{\lambda+1} m$ is a fundamental discriminant (note. which includes 1$)$, then for each positive integer $n$ with $(-1)^{\lambda} n \equiv 0,1$ $(\bmod 4)$ we have

$$
b_{\lambda}(-m ; n)=\frac{2(-1)^{[(\lambda+1) / 2]} n^{\frac{\lambda}{2}-\frac{1}{2}}}{m^{\frac{\lambda}{2}}} \cdot \operatorname{Tr}_{(-1)^{\lambda+1} m}\left(\mathfrak{F}_{\lambda} ; n\right) .
$$

REMARK 48. A version of Theorem 13.3 holds for integers $\lambda \leq 0$. This follows from a duality (see Theorem 1.1 of [54]) of Fourier expansions. Suppose that $\lambda \geq 1$, and that $m$ is a positive integer for which $(-1)^{\lambda+1} m \equiv 0,1$ $(\bmod 4)$. For every positive integer $n$ with $(-1)^{\lambda} n \equiv 0,1(\bmod 4)$, this duality implies that

$$
b_{\lambda}(-m ; n)=-b_{1-\lambda}(-n ; m) .
$$

Example 13.4. For $\lambda=1$, Theorem 13.3 relates $b_{1}(-m ; n)$ to traces and twisted traces of $\mathfrak{F}_{1}(z)=\frac{1}{2}(j(z)-744)$. These are Theorems 1 and 6 of Zagier's paper [212].

Theorem 13.3 is obtained by reformulating, as traces of singular moduli, exact expressions for the coefficients $b_{\lambda}(-m ; n)$. The proof follows from the classical fact that certain half-integral weight Kloosterman sums are essentially Salié sums. To define these sums, suppose that $0 \neq D_{1} \equiv 0,1$ $(\bmod 4)$. If $\lambda$ is an integer, $D_{2} \neq 0$ is an integer for which $(-1)^{\lambda} D_{2} \equiv 0,1$ $(\bmod 4)$, and $N$ is a positive multiple of 4 , then define the generalized Salié sum $S_{\lambda}\left(D_{1}, D_{2}, N\right)$ by

$$
\begin{aligned}
S_{\lambda}\left(D_{1}, D_{2}, N\right):= & \sum_{\substack{x \\
(\bmod N)}} \chi_{D_{1}}\left(\frac{N}{4}, x, \frac{x^{2}-(-1)^{\lambda} D_{1} D_{2}}{N}\right) \\
& \times e\left(\frac{2 x}{N}\right),
\end{aligned}
$$


K. ONO

where $\chi_{D_{1}}(a, b, c)$, for a binary quadratic form $Q=[a, b, c]$, is given by

$$
\chi_{D_{1}}(a, b, c):= \begin{cases}0 & \text { if }\left(a, b, c, D_{1}\right)>1 \\ \left(\frac{D_{1}}{r}\right) & \text { if }\left(a, b, c, D_{1}\right)=1 \text { and } Q \text { represents } \\ & \quad r \text { with }\left(r, D_{1}\right)=1 .\end{cases}
$$

REMARK 49. If $D_{1}=1$, then $\chi_{D_{1}}$ is trivial. Therefore, if $(-1)^{\lambda} D_{2} \equiv 0,1$ $(\bmod 4)$, then

$$
S_{\lambda}\left(1, D_{2}, N\right)=\sum_{\substack{x \\ x^{2} \equiv(-1)^{\lambda} D_{2}(\bmod N)}} e\left(\frac{2 x}{N}\right) .
$$

Half-integral weight Kloosterman sums are essentially equal to such Salié sums, a fact which plays a fundamental role throughout the theory of halfintegral weight modular forms. The following proposition is due to Kohnen (see Proposition 5 of [136]).

Proposition 13.5. Suppose that $N$ is a positive multiple of 4 . If $\lambda$ is an integer, and $D_{1}$ and $D_{2}$ are non-zero integers for which $D_{1},(-1)^{\lambda} D_{2} \equiv 0,1$ $(\bmod 4)$, then

$$
N^{-\frac{1}{2}}\left(1-(-1)^{\lambda} i\right)\left(1+\delta_{\text {odd }}(N / 4)\right) \cdot K_{\lambda}\left((-1)^{\lambda} D_{1}, D_{2}, N\right)=S_{\lambda}\left(D_{1}, D_{2}, N\right) .
$$

As a consequence, we may rewrite the formulas for the $b_{\lambda}(-m ; n)$ using Salié sums. The following proposition describes these Salié sums as Poincarétype series over CM points.

Proposition 13.6. Suppose that $\lambda$ is an integer, and that $D_{1}$ is a fundamental discriminant. If $D_{2}$ is a non-zero integer for which $(-1)^{\lambda} D_{2} \equiv 0,1$ $(\bmod 4)$ and $(-1)^{\lambda} D_{1} D_{2}<0$, then for every positive integer a we have

$$
S_{\lambda}\left(D_{1}, D_{2}, 4 a\right)=2 \sum_{Q \in \mathcal{Q}_{\left|D_{1} D_{2}\right|} / \Gamma} \frac{\chi_{D_{1}}(Q)}{\omega_{Q}} \sum_{\substack{A \in \Gamma \infty \backslash S L_{2}(\mathbb{Z}) \\ \operatorname{Im}\left(A \tau_{Q}\right)=\frac{\sqrt{\left|D_{1} D_{2}\right|}}{2 a}}} e\left(-\operatorname{Re}\left(A \tau_{Q}\right)\right) .
$$

Proof. For every integral binary quadratic form

$$
Q(x, y)=a x^{2}+b x y+c y^{2}
$$

of discriminant $(-1)^{\lambda} D_{1} D_{2}$, let $\tau_{Q} \in \mathbb{H}$ be as before. Clearly $\tau_{Q}$ is equal to

$$
\tau_{Q}=\frac{-b+i \sqrt{\left|D_{1} D_{2}\right|}}{2 a}
$$

and the coefficient $b$ of $Q$ solves the congruence

$$
b^{2} \equiv(-1)^{\lambda} D_{1} D_{2} \quad(\bmod 4 a)
$$

Conversely, every solution of (13.14) corresponds to a quadratic form with an associated CM point thereby providing a one-to-one correspondence between the solutions of

$$
b^{2}-4 a c=(-1)^{\lambda} D_{1} D_{2} \quad(a, b, c \in \mathbb{Z}, a, c>0)
$$


and the points of the orbits

$$
\bigcup_{Q}\left\{A \tau_{Q}: A \in \mathrm{SL}_{2}(\mathbb{Z}) / \Gamma_{\tau_{Q}}\right\}
$$

where $\Gamma_{\tau_{Q}}$ denotes the isotropy subgroup of $\tau_{Q}$ in $\mathrm{SL}_{2}(\mathbb{Z})$, and where $Q$ varies over the representatives of $\mathcal{Q}_{\left|D_{1} D_{2}\right|} / \Gamma$. The group $\Gamma_{\infty}$ preserves the imaginary part of such a CM point $\tau_{Q}$, and preserves (13.14). However, it does not preserve the middle coefficient $b$ of the corresponding quadratic forms modulo $4 a$. It identifies the congruence classes $b, b+2 a(\bmod 4 a)$ appearing in the definition of $S_{\lambda}\left(D_{1}, D_{2}, 4 a\right)$. Since $\chi_{D_{1}}(Q)$ is fixed under the action of $\Gamma_{\infty}$, the corresponding summands for such pairs of congruence classes are equal. Proposition 13.6 follows since $\# \Gamma_{\tau_{Q}}=2 \omega_{Q}$, and since both $\Gamma_{\tau_{Q}}$ and $\Gamma_{\infty}$ contain the negative identity matrix.

Sketch of the Proof of Theorem 13.3. Here we prove the cases where $\lambda \geq 2$. The argument when $\lambda=1$ is identical. For $\lambda \geq 2$, the exact formula for $b_{\lambda}(-m ; n)$ is

$$
\begin{aligned}
b_{\lambda}(-m ; n)= & (-1)^{[(\lambda+1) / 2]} \pi \sqrt{2}(n / m)^{\frac{\lambda}{2}-\frac{1}{4}}\left(1-(-1)^{\lambda} i\right) \\
& \times \sum_{c \equiv 0}\left(1+\delta_{\text {odd }}(c / 4)\right) \frac{K_{\lambda}(-m, n, c)}{c} \cdot I_{\lambda-\frac{1}{2}}\left(\frac{4 \pi \sqrt{m n}}{c}\right) .
\end{aligned}
$$

Using Proposition 13.5, where $D_{1}=(-1)^{\lambda+1} m$ and $D_{2}=n$, for integers $N=c$ which are positive multiples of 4 , we have

$$
c^{-\frac{1}{2}}\left(1-(-1)^{\lambda} i\right)\left(1+\delta_{\text {odd }}(c / 4)\right) \cdot K_{\lambda}(-m, n, c)=S_{\lambda}\left((-1)^{\lambda+1} m, n, c\right) .
$$

These identities, combined with the change of variable $c=4 a$, give

$$
\begin{aligned}
b_{\lambda}(-m ; n)= & \frac{(-1)^{[(\lambda+1) / 2]} \pi}{\sqrt{2}}(n / m)^{\frac{\lambda}{2}-\frac{1}{4}} \sum_{a=1}^{\infty} \frac{S_{\lambda}\left((-1)^{\lambda+1} m, n, 4 a\right)}{\sqrt{a}} \\
& \cdot I_{\lambda-\frac{1}{2}}\left(\frac{\pi \sqrt{m n}}{a}\right) .
\end{aligned}
$$

Using Proposition 13.6, this becomes

$$
\begin{gathered}
b_{\lambda}(-m ; n)=\frac{2(-1)^{[(\lambda+1) / 2]} \pi}{\sqrt{2}}(n / m)^{\frac{\lambda}{2}-\frac{1}{4}} \sum_{\substack{Q \in \mathcal{Q}_{n m} / \Gamma \\
\omega_{Q}}} \frac{\chi_{(-1)^{\lambda+1} m}(Q)}{\omega_{Q}} \\
\sum_{a=1}^{\infty} \sum_{\substack{A \in \Gamma_{\infty} \backslash \mathrm{SL}_{2}(\mathbb{Z}) \\
\operatorname{Im}\left(A \tau_{Q}\right)=\frac{\sqrt{m n}}{2 a}}} \frac{I_{\lambda-\frac{1}{2}}\left(2 \pi \operatorname{Im}\left(A \tau_{Q}\right)\right)}{\sqrt{a}} \cdot e\left(-\operatorname{Re}\left(A \tau_{Q}\right)\right) .
\end{gathered}
$$


The definition of $\mathfrak{F}_{\lambda}(z)$ in (13.10), combined with the obvious change of variable relating $1 / \sqrt{a}$ to $\operatorname{Im}\left(A \tau_{Q}\right)^{\frac{1}{2}}$, gives

$$
\begin{aligned}
& b_{\lambda}(-m ; n)=\frac{2(-1)^{[(\lambda+1) / 2]} n^{\frac{\lambda}{2}-\frac{1}{2}}}{m^{\frac{\lambda}{2}}} \cdot \pi \sum_{Q \in \mathcal{Q}_{n m} / \Gamma} \frac{\chi_{(-1)^{\lambda+1} m}(Q)}{\omega_{Q}} \\
& \sum_{A \in \Gamma_{\infty} \backslash \mathrm{SL}_{2}(\mathbb{Z})} \operatorname{Im}\left(A \tau_{Q}\right)^{\frac{1}{2}} \cdot I_{\lambda-\frac{1}{2}}\left(2 \pi \operatorname{Im}\left(A \tau_{Q}\right)\right) e\left(-\operatorname{Re}\left(A \tau_{Q}\right)\right) \\
&=\frac{2(-1)^{[(\lambda+1) / 2]} n^{\frac{\lambda}{2}-\frac{1}{2}}}{m^{\frac{\lambda}{2}}} \cdot \operatorname{Tr}_{(-1)^{\lambda+1} m}\left(\mathfrak{F}_{\lambda} ; n\right) .
\end{aligned}
$$

13.2. The "24-Theorem". Exact formulas for traces of singular moduli can lead to nice number theoretic consequences. Here we mention one such application which is related to the classical observation that

$$
e^{\pi \sqrt{163}}=262537412640768743.9999999999992 \ldots
$$

is nearly an integer. To make this precise, we recall some classical facts. A primitive positive definite binary quadratic form $Q$ is reduced if $|B| \leq A \leq$ $C$, and $B \geq 0$ if either $|B|=A$ or $A=C$. If $-d<-4$ is a fundamental discriminant, then there are $H(d)$ reduced forms with discriminant $-d$. The set of such reduced forms, say $\mathcal{Q}_{d}^{\text {red }}$, is a complete set of representatives for $\mathcal{Q}_{d} / \mathrm{SL}_{2}(\mathbb{Z})$. Moreover, each such reduced form has $1 \leq A \leq \sqrt{d / 3}$ (see page 29 of $[\mathbf{8 2}]$ ), and has the property that $\tau_{Q}$ lies in the usual fundamental domain for the action of $\mathrm{SL}_{2}(\mathbb{Z})$

$$
\mathcal{F}=\left\{-\frac{1}{2} \leq \operatorname{Re}(z)<\frac{1}{2} \text { and }|z|>1\right\} \cup\left\{-\frac{1}{2} \leq \operatorname{Re}(z) \leq 0 \text { and }|z|=1\right\} .
$$

Since $J_{1}(z):=j(z)-744=q^{-1}+196884 q+\cdots$, it follows that if $G^{\text {red }}(d)$ is defined by

$$
G^{\mathrm{red}}(d)=\sum_{Q=(A, B, C) \in \mathcal{Q}_{d}^{\mathrm{red}}} e^{\pi B i / A} \cdot e^{\pi \sqrt{d} / A},
$$

then $\operatorname{Tr}(d)-G^{\text {red }}(d)$ is "small", where $\operatorname{Tr}(d):=\operatorname{Tr}\left(J_{1} ; d\right)$. In other words, $q^{-1}$ provides a good approximation for $J_{1}(z)$ for most points $z$. This is illustrated by $(13.15)$ where $H(163)=1$.

It is natural to investigate the "average value"

$$
\frac{\operatorname{Tr}(d)-G^{\mathrm{red}}(d)}{H(d)}
$$

which for $d=163$ is $-0.0000000000008 \ldots$. Armed with the exact formulas for $\operatorname{Tr}(d)$, it turns out that a uniform picture emerges for a slightly perturbed 
average, one including some non-reduced quadratic forms. For each positive integer $A$, let $\mathcal{Q}_{A, d}^{\text {old }}$ denote the set

$$
\mathcal{Q}_{A, d}^{\text {old }}=\left\{Q=(A, B, C) \text { : non-reduced with } D_{Q}=-d \text { and }|B| \leq A\right\} .
$$

Define $G^{\text {old }}(d)$ by

$$
G^{\text {old }}(d)=\sum_{\substack{\sqrt{d} / 2 \leq A \leq \sqrt{d / 3} \\ Q \in \mathcal{Q}_{A, d}^{\text {old }}}} e^{\pi B i / A} \cdot e^{\pi \sqrt{d} / A} .
$$

The non-reduced forms $Q$ contributing to $G^{\text {old }}(d)$ are those primitive discriminant $-d$ forms for which $\tau_{Q}$ is in the bounded region obtained by connecting the two endpoints of the lower boundary of $\mathcal{F}$ with a horizontal line. Since $\operatorname{Tr}(d)$ is subexponential in $|d|$ and $H(d) \ll|d|^{\frac{1}{2}+\epsilon}$, the following numerics are quite surprising:

$$
\frac{\operatorname{Tr}(d)-G^{\mathrm{red}}(d)-G^{\mathrm{old}}(d)}{H(d)}= \begin{cases}-24.672 \ldots & \text { if } d=1931 \\ -24.483 \ldots & \text { if } d=2028 \\ -23.458 \ldots & \text { if } d=2111\end{cases}
$$

Recently, Duke has proved [87] a result which implies the following theorem.

THEOREM 13.7. As -d ranges over negative fundamental discriminants, we have

$$
\lim _{-d \rightarrow-\infty} \frac{\operatorname{Tr}(d)-G^{\mathrm{red}}(d)-G^{\mathrm{old}}(d)}{H(d)}=-24 .
$$

Here we explain the source of -24 in the limit

$$
\lim _{-d \rightarrow-\infty} \frac{\operatorname{Tr}(d)-G^{\mathrm{red}}(d)-G^{\mathrm{old}}(d)}{H(d)}=-24 .
$$

Using Theorem 13.3 and Propositions 13.5 and 13.6, it is not difficult to reformulate the exact formulas for $\operatorname{Tr}(d)$ to read

$$
\operatorname{Tr}(d)=-24 H(d)+\sum_{\substack{c>0 \\ c \equiv 0(4)}} S(d, c) \sinh (4 \pi \sqrt{d} / c),
$$

where $S(d, c)$ is the Salié sum

$$
S(d, c)=\sum_{x^{2} \equiv-d \quad(\bmod c)} e(2 x / c) .
$$

As a consequence, the " 24 Theorem" is equivalent to the assertion that

$$
\sum_{\substack{c>\sqrt{d / 3} \\ c \equiv 0(4)}} S(d, c) \sinh \left(\frac{4 \pi}{c} \sqrt{d}\right)=o(H(d)) .
$$


This follows from the fact the sum over $c \leq \sqrt{d / 3}$ is essentially $G^{\text {red }}(d)+$ $G^{\text {old }}(d)$. The sinh factor contributes the size of $q^{-1}$ in the Fourier expansion of a singular modulus, and the summands in the Kloosterman sum provide the corresponding "angles". The contribution $G^{\text {old }}(d)$ arises from the fact that the Kloosterman sum cannot distinguish between reduced and nonreduced forms. In view of Siegel's theorem that $H(d) \gg_{\epsilon} d^{\frac{1}{2}-\epsilon},(13.20)$ follows from a bound for such sums of the form $\ll d^{\frac{1}{2}-\gamma}$, for some $\gamma>$ 0 . Such bounds are implicit in Duke's proof of this result [87], and more generally in his famous work on bounding coefficients of half-integral weight cusp forms $[\mathbf{8 6}]$.

\section{Borcherds products}

Here we describe work of the author and Bruinier [67] on Borcherds products. To describe the context of this work, we first recall a famous result of Borcherds on infinite product expansions of modular forms possessing a Heegner divisor.

14.1. The "classical" Borcherds products. We begin by recalling that $\Delta(z)$, the unique normalized weight 12 cusp form with respect to $\mathrm{SL}_{2}(\mathbb{Z})$, has a Fourier expansion given by the infinite product

$$
\Delta(z)=q \prod_{n=1}^{\infty}\left(1-q^{n}\right)^{24}
$$

Most modular forms do not possess simple infinite product expansions. It was widely believed that such elegant product expansions only belong to the domain of those modular forms whose divisors are supported at cusps, such as the modular units (for example, see [139]).

Then in the 1990s, Borcherds $[\mathbf{4 2 , 4 3 , 4 4}]$ provided a striking description for the exponents in the naive infinite product expansions of a much larger class of modular forms, those modular forms possessing a Heegner divisor. For example, let $c(n)$ denote the integer exponents one obtains by expressing $E_{4}(z)$ as an infinite product:

$E_{4}(z)=1+240 \sum_{n=1}^{\infty} \sum_{d \mid n} d^{3} q^{n}=(1-q)^{-240}\left(1-q^{2}\right)^{26760} \cdots=\prod_{n=1}^{\infty}\left(1-q^{n}\right)^{c(n)}$.

Borcherds's theory implies that there is a weight $1 / 2$ meromorphic modular form

$$
G(z)=\sum_{n \geq-3} b(n) q^{n}=q^{-3}+4-240 q+26760 q^{4}+\cdots-4096240 q^{9}+\ldots
$$

with the property that $c(n)=b\left(n^{2}\right)$ for every positive integer $n$. Notice that the product in (14.1) also satisfies this phenomenon, where the product 
exponents are the coefficients of the weight $1 / 2$ modular form

$$
12 \Theta_{0}(z)=12 \sum_{n \in \mathbb{Z}} q^{n^{2}}=12+24 q+24 q^{4}+24 q^{9}+\cdots .
$$

These two examples illustrate his general result that a modular form on $\mathrm{SL}_{2}(\mathbb{Z})$ with a Heegner divisor has an infinite product expansion where the exponents are coefficients of certain weight $1 / 2$ weakly holomorphic modular forms.

REMARK 50. The work of Borcherds $[\mathbf{4 2}, \mathbf{4 3}, \mathbf{4 4}]$ is given in the more general context of automorphic forms on orthogonal groups.

REMARK 51. It is possible to derive explicit formulas for the exponents in the infinite product expansions of generic modular forms, not just those with a Heegner divisor (for example, see [66]). However, these formulas are in general quite complicated.

We now briefly recall the most classical case of this work of Borcherds. We shall refer to a complex number $\tau \in \mathbb{H}$ of the form

$$
\tau=\frac{-b+\sqrt{b^{2}-4 a c}}{2 a}
$$

with $a, b, c \in \mathbb{Z}, \operatorname{gcd}(a, b, c)=1$, and $b^{2}-4 a c<0$ as a $C M$ point, and we denote its discriminant by the integer $d_{\tau}:=b^{2}-4 a c$. A meromorphic modular form $f(z)$ on $\mathrm{SL}_{2}(\mathbb{Z})$ is said to have a Heegner divisor if its zeros and poles are supported at the cusp at infinity and at CM points.

To state Borcherds's results, we require a special sequence of weight $1 / 2$ modular forms in $\mathcal{M}_{\frac{1}{2}}^{!}(4)$. These forms will be distinguished by their $q$-expansions. For each nonnegative integer $d \equiv 0,3(\bmod 4)$ let $f_{d}(z) \in$ $\mathcal{M}_{\frac{1}{2}}^{!}(4)$ be the unique modular form with a Fourier expansion of the form

$$
f_{d}(z)=q^{-d}+\sum_{D>0} A(D, d) q^{D} .
$$

That these forms are well defined follows from Lemma 14.2 of [43]. Moreover, they form a basis of $\mathcal{M}_{\frac{1}{2}}^{!}(4)$.

The form $f_{0}(z)$ is the classical Jacobi theta-function

$$
f_{0}(z)=\Theta_{0}(z)=1+2 q+2 q^{4}+2 q^{9}+2 q^{16}+\cdots,
$$

and the form $f_{3}(z)$ is given by the expression

$$
\begin{aligned}
f_{3}(z) & =\frac{D\left(\Theta_{0}(z)\right) E_{10}(4 z)}{2 \Delta(4 z)}-\frac{\Theta_{0}(z)\left(D\left(E_{10}(z)\right) \mid V(4)\right)}{10 \Delta(4 z)}-\frac{152}{5} \Theta_{0}(z) \\
& =q^{-3}-248 q+26752 q^{4}-85995 q^{5}+\cdots
\end{aligned}
$$

where $D:=q \cdot \frac{d}{d q}$. 
REMARK 52. It is straightforward to inductively compute the $f_{d}(z)$ using $f_{0}(z)$ and $f_{3}(z)$. To compute $f_{d}(z)$ for $d \geq 4$, one computes $f_{d-4}(z) j(4 z)$, and then iteratively subtracts multiples of those $f_{j}(z)$ with $0 \leq j \leq d-4$.

For completeness, we include the initial terms of the first few $f_{d}(z)$ below.

$$
\begin{aligned}
f_{0}(z)= & 1+2 q+2 q^{4}+2 q^{9}+\cdots, \\
f_{3}(z)= & q^{-3}-248 q+26752 q^{4}-85995 q^{5}+1707264 q^{8}-4096248 q^{9}+\cdots, \\
f_{4}(z)= & q^{-4}+492 q+143376 q^{4}+565760 q^{5}+18473000 q^{8} \\
& +51180012 q^{9}+\cdots, \\
f_{7}(z)= & q^{-7}-4119 q+8288256 q^{4}-52756480 q^{5}+\cdots, \\
f_{8}(z)= & q^{-8}+7256 q+26124256 q^{4}+190356480 q^{5}+\cdots .
\end{aligned}
$$

Using these forms, we may now state Borcherds's famous theorem on the infinite product expansion of those meromorphic forms on $\mathrm{SL}_{2}(\mathbb{Z})$ possessing a Heegner divisor. Let $\mathcal{M}_{H}$ denote the set of integer weight meromorphic modular forms on $\mathrm{SL}_{2}(\mathbb{Z})$ with a Heegner divisor, integer coefficients, and leading coefficient 1 . Obviously, $\mathcal{M}_{H}$ is closed under multiplication.

If $H(-n)$ denotes the usual Hurwitz class number of discriminant $-n$, then define $\widetilde{H}(z)$ by

$$
\begin{aligned}
\widetilde{H}(z): & =-\frac{1}{12}+\sum_{1<n \equiv 0,3} H(-n) q^{n} \\
& =-\frac{1}{12}+q^{3} / 3+q^{4} / 2+q^{7}+q^{8}+q^{11}+4 q^{12} / 3+\cdots .
\end{aligned}
$$

If $f(z)=\sum_{n \geq n_{0}} A(n) q^{n} \in \mathcal{M}_{\frac{1}{2}}^{!}(4)$, then define $\Psi(f(z))$ by

$$
\Psi(f(z)):=q^{-h} \prod_{n=1}^{\infty}\left(1-q^{n}\right)^{A\left(n^{2}\right)},
$$

where $h$ is the constant term of $f(z) \widetilde{H}(z)$. Borcherds $[42]$ proved the following theorem.

TheOREM 14.1. The map $\Psi$ satisfies

$$
\Psi: \mathcal{M}_{\frac{1}{2}}^{!}(4) \longrightarrow \mathcal{M}_{H}
$$

Furthermore, it is an isomorphism. The weight of $\Psi(f)$ is $A(0)$, and the multiplicity of the zero of $\Psi(f)$ at a CM point of discriminant $D<0$ is

$$
\sum_{n>0} A\left(D n^{2}\right)
$$

REMARK 53. The isomorphism $\Psi$ respects the additive structure of $\mathcal{M}_{\frac{1}{2}}^{!}(4)$ and the multiplicative structure of $\mathcal{M}_{H}$. 
EXAmPle 14.2. If $f(z)=12 f_{0}(z)=12 \Theta_{0}(z)$, then

$$
f(z)=\sum_{n=0}^{\infty} A(n) q^{n}=12+24 q+24 q^{4}+24 q^{9}+24 q^{16}+\cdots .
$$

Therefore, it follows that

$$
\Psi(f(z))=q \prod_{n=1}^{\infty}\left(1-q^{n}\right)^{24}
$$

Obviously, $\Psi(f(z))$ is the classical $\Delta$-function. Its weight is $A(0)=12$, and its divisor is supported at the cusp at infinity.

EXAMPLE 14.3. If $f(z)=3 f_{3}(z)$, then

$$
\begin{aligned}
f(z)= & \sum_{n=-3}^{\infty} A(n) q^{n}=3 q^{-3}-744 q+80256 q^{4}-257985 q^{5}+5121792 q^{8} \\
& -12288744 q^{9}+\cdots .
\end{aligned}
$$

By Theorem 14.1, it follows that $\Psi(f(z))$ is a weight $A(0)=0$ meromorphic modular form whose divisor is supported at the cusp at infinity and a triple zero at $\omega=e^{2 \pi i / 3}$. Therefore, it follows that $\Psi(f(z))$ must be $j(z)$, and this is confirmed by

$$
\begin{aligned}
\Psi(f(z)) & =q^{-1}(1-q)^{-744}\left(1-q^{2}\right)^{80256}\left(1-q^{3}\right)^{-12288744} \ldots \\
& =q^{-1}+744+196884 q+21493760 q^{3}+\cdots .
\end{aligned}
$$

EXAMPLE 14.4. If $f(z)=4 f_{0}(z)+f_{3}(z)$, then

$$
\begin{aligned}
f(z)= & \sum_{n=-3}^{\infty} A(n) q^{n}=q^{-3}+4-240 q+26760 q^{4} \\
& -85995 q^{5}+\cdots-4096240 q^{9}+\cdots .
\end{aligned}
$$

Theorem 14.1 implies that $\Psi(f(z))$ is a holomorphic modular form of weight 4 with leading coefficient 1 . Therefore, it must be that $\Psi(f(z))=E_{4}(z)$. This explains (14.2).

14.2. Harmonic Maass forms and generalized Borcherds products. Motivated by questions related to derivatives of modular $L$-functions, the author and Bruinier [67] derived Borcherds-type products using harmonic Maass forms. These results are quite technical, and a thorough treatment would occupy more space than is warranted in this expository paper. For brevity, we will be content with a brief indication of the flavor of these results, followed by two illustrative examples.

These results are phrased in terms of vector-valued weight $1 / 2$ harmonic Maass forms. Let $(V, Q)$ be a non-degenerate rational quadratic space of signature $\left(b^{+}, b^{-}\right)$, and let $L \subset V$ be an even lattice with dual $L^{\prime}$. The discriminant group $L^{\prime} / L$, together with the $\mathbb{Q} / \mathbb{Z}$-valued quadratic form induced by $Q$, is called the discriminant form of the lattice $L$. 
As usual, we let $\mathrm{Mp}_{2}(\mathbb{R})$ denote the metaplectic two-fold cover of $\mathrm{SL}_{2}(\mathbb{R})$. The elements of this group are pairs $(M, \phi(\tau))$, where $M=\left(\begin{array}{ll}a & b \\ c & d\end{array}\right) \in \mathrm{SL}_{2}(\mathbb{R})$ and $\phi: \mathbb{H} \rightarrow \mathbb{C}$ is a holomorphic function with $\phi(\tau)^{2}=c \tau+d$. The group law is defined by

$$
(M, \phi(\tau))\left(M^{\prime}, \phi^{\prime}(\tau)\right)=\left(M M^{\prime}, \phi\left(M^{\prime} \tau\right) \phi^{\prime}(\tau)\right) .
$$

We denote the integral metaplectic group, the inverse image of $\Gamma:=\mathrm{SL}_{2}(\mathbb{Z})$ under the covering map, by $\widetilde{\Gamma}:=\mathrm{Mp}_{2}(\mathbb{Z})$. It is well known that $\widetilde{\Gamma}$ is generated by $T:=\left(\left(\begin{array}{ll}1 & 1 \\ 0 & 1\end{array}\right), 1\right)$, and $S:=\left(\left(\begin{array}{cc}0 & -1 \\ 1 & 0\end{array}\right), \sqrt{\tau}\right)$. One has the relations $S^{2}=(S T)^{3}=Z$, where $Z:=\left(\left(\begin{array}{cc}-1 & 0 \\ 0 & -1\end{array}\right), i\right)$ is the standard generator of the center of $\widetilde{\Gamma}$. We let $\widetilde{\Gamma}_{\infty}:=\langle T\rangle \subset \widetilde{\Gamma}$.

We now recall the Weil representation associated with the discriminant form $L^{\prime} / L$ (for example, see $[\mathbf{4 3}],[6 \mathbf{6 2}]$ ). It is a representation of $\widetilde{\Gamma}$ on the group algebra $\mathbb{C}\left[L^{\prime} / L\right]$. We denote the standard basis elements of $\mathbb{C}\left[L^{\prime} / L\right]$ by $\mathfrak{e}_{h}, h \in L^{\prime} / L$, and write $\langle\cdot, \cdot\rangle$ for the standard scalar product (antilinear in the second entry) such that $\left\langle\mathfrak{e}_{h}, \mathfrak{e}_{h^{\prime}}\right\rangle=\delta_{h, h^{\prime}}$. The Weil representation $\rho_{L}$ associated with the discriminant form $L^{\prime} / L$ is the unitary representation of $\widetilde{\Gamma}$ on $\mathbb{C}\left[L^{\prime} / L\right]$ defined by

$$
\begin{aligned}
\rho_{L}(T)\left(\mathfrak{e}_{h}\right) & :=e\left(h^{2} / 2\right) \mathfrak{e}_{h}, \\
\rho_{L}(S)\left(\mathfrak{e}_{h}\right) & :=\frac{e\left(\left(b^{-}-b^{+}\right) / 8\right)}{\sqrt{\left|L^{\prime} / L\right|}} \sum_{h^{\prime} \in L^{\prime} / L} e\left(-\left(h, h^{\prime}\right)\right) \mathfrak{e}_{h^{\prime}} .
\end{aligned}
$$

If $f: \mathbb{H} \rightarrow \mathbb{C}\left[L^{\prime} / L\right]$ is a function, we write $f=\sum_{\lambda \in L^{\prime} / L} f_{h} \mathfrak{e}_{h}$ for its decomposition in components with respect to the standard basis of $\mathbb{C}\left[L^{\prime} / L\right]$. Let $k \in \frac{1}{2} \mathbb{Z}$, and let $M_{k, \rho_{L}}^{!}$denote the space of $\mathbb{C}\left[L^{\prime} / L\right]$-valued weakly holomorphic modular forms of weight $k$ and type $\rho_{L}$ for the group $\widetilde{\Gamma}$.

Now assume that $k \leq 1$. A twice continuously differentiable function $f: \mathbb{H} \rightarrow \mathbb{C}\left[L^{\prime} / L\right]$ is called a vector-valued harmonic Maass form of weight $k$ with respect to $\widetilde{\Gamma}$ and $\rho_{L}$ if it satisfies:

(i) $f(M \tau)=\phi(\tau)^{2 k} \rho_{L}(M, \phi) f(\tau)$ for all $(M, \phi) \in \widetilde{\Gamma}$;

(ii) $\Delta_{k} f=0$;

(iii) We have that

$$
f(\tau)=P_{f}(\tau)+O\left(e^{-\varepsilon v}\right),
$$

as $v \rightarrow \infty$, for some Fourier polynomial

$$
P_{f}(\tau)=\sum_{h \in L^{\prime} / L} \sum_{\substack{n \in \mathbb{Z}+Q(h) \\-\infty \ll n \leq 0}} c^{+}(n, h) e(n \tau) \mathfrak{e}_{h}
$$

and some $\varepsilon>0$.

We write $H_{k, \rho_{L}}$ for the vector space of such harmonic Maass forms. 
Generalizing Lemma 7.2, where the modular variable is now $\tau=u+i v$, we have that any $f \in H_{k, \rho_{L}}$ decomposes as $f=f^{+}+f^{-}$, where

$$
\begin{aligned}
f^{+}(\tau) & =\sum_{h \in L^{\prime} / L} \sum_{\substack{n \in \mathbb{Q} \\
n \gg-\infty}} c^{+}(n, h) e(n \tau) \mathfrak{e}_{h}, \\
f^{-}(\tau) & =\sum_{h \in L^{\prime} / L} \sum_{\substack{n \in \mathbb{Q} \\
n<0}} c^{-}(n, h) W(2 \pi n v) e(n \tau) \mathfrak{e}_{h},
\end{aligned}
$$

and $W(x)=W_{k}(x):=\int_{-2 x}^{\infty} e^{-t} t^{-k} d t=\Gamma(1-k, 2|x|)$ for $x<0$.

In [67], the theory of theta liftings is applied to such harmonic Maass forms to obtain Borcherds products with twisted Heegner divisors on $X_{0}(N)$. To this end, let $N$ be a positive integer. We consider the rational quadratic space

$$
V:=\left\{X \in \operatorname{Mat}_{2}(\mathbb{Q}): \operatorname{tr}(X)=0\right\}
$$

with the quadratic form $Q(X):=-N \operatorname{det}(X)$. We let $L$ be the lattice

$$
L:=\left\{\left(\begin{array}{cc}
b & -a / N \\
c & -b
\end{array}\right): \quad a, b, c \in \mathbb{Z}\right\} .
$$

Then the dual lattice is given by

$$
L^{\prime}:=\left\{\left(\begin{array}{cc}
b / 2 N & -a / N \\
c & -b / 2 N
\end{array}\right): \quad a, b, c \in \mathbb{Z}\right\} .
$$

We identify $L^{\prime} / L$ with $\mathbb{Z} / 2 N \mathbb{Z}$. Here the quadratic form on $L^{\prime} / L$ is identified with the quadratic form $x \mapsto x^{2}$ on $\mathbb{Z} / 2 N \mathbb{Z}$.

If $D \in \mathbb{Z}$, let $L_{D}$ be the set of vectors $X \in L^{\prime}$ with $Q(X)=D / 4 N$. Notice that $L_{D}$ is empty unless $D$ is a square modulo $4 N$. For $r \in L^{\prime} / L$ with $r^{2} \equiv D(\bmod 4 N)$ we define

$$
L_{D, r}:=\left\{X \in L^{\prime}: \quad Q(X)=D / 4 N \text { and } X \equiv r \quad(\bmod L)\right\} .
$$

If $X=\left(\begin{array}{cc}b / 2 N & -a / N \\ c & -b / 2 N\end{array}\right) \in L_{D, r}$, then the matrix

$$
\psi(X):=\left(\begin{array}{cc}
a & b / 2 \\
b / 2 & N c
\end{array}\right)=X\left(\begin{array}{cc}
0 & N \\
-N & 0
\end{array}\right)
$$

defines an integral binary quadratic form of discriminant $D=b^{2}-4 N a c=$ $4 N Q(X)$ with $b \equiv r(\bmod 2 N)$. We have that $\Gamma_{0}(N)$ acts on $L_{D, r}$, and the number of orbits of $L_{D, r}$ is finite if $D \neq 0$.

Let $\Delta \in \mathbb{Z}$ be a fundamental discriminant and $r \in \mathbb{Z}$ such that $\Delta \equiv r^{2}$ $(\bmod 4 N)$. Following $[\mathbf{1 0 7}]$, we define a generalized genus character for 
$\lambda=\left(\begin{array}{cc}b / 2 N & -a / N \\ c & -b / 2 N\end{array}\right) \in L^{\prime}$ by putting

$$
\chi_{\Delta}(\lambda)=\chi_{\Delta}([a, b, N c]):= \begin{cases}\left(\frac{\Delta}{n}\right), & \text { if } \Delta \mid b^{2}-4 N a c \text { and } \\ & \left(b^{2}-4 N a c\right) / \Delta \text { is a square modulo } 4 N \\ & \text { and } \operatorname{gcd}(a, b, c, \Delta)=1, \\ & \text { otherwise }\end{cases}
$$

Here $[a, b, N c]$ is the integral binary quadratic form corresponding to $\lambda$, and $n$ is any integer prime to $\Delta$ represented by one of the quadratic forms $\left[N_{1} a, b, N_{2} c\right]$ with $N_{1} N_{2}=N$ and $N_{1}, N_{2}>0$.

The cusps of $\Gamma_{0}(N)$ correspond to $\Gamma_{0}(N)$-classes of primitive isotropic vectors in $L$. Here we let $\ell, \ell^{\prime} \in L$ be the isotropic vectors

$$
\ell=\left(\begin{array}{cc}
0 & 1 / N \\
0 & 0
\end{array}\right), \quad \ell^{\prime}=\left(\begin{array}{ll}
0 & 0 \\
1 & 0
\end{array}\right) .
$$

The 1-dimensional lattice

$$
K=L \cap \ell^{\perp} \cap \ell^{\perp}
$$

is positive definite. We find that $L$ splits into

$$
L=K \oplus \mathbb{Z} \ell^{\prime} \oplus \mathbb{Z} \ell
$$

and $K^{\prime} / K \cong L^{\prime} / L$. Then we have $K=\mathbb{Z}\left(\begin{array}{cc}1 & 0 \\ 0 & -1\end{array}\right)$. For $\lambda \in K \otimes \mathbb{R}$, we write $\lambda>0$ if $\lambda$ is a positive multiple of $\left(\begin{array}{cc}1 & 0 \\ 0 & -1\end{array}\right)$.

We now define twisted Heegner divisors on the modular curve $X_{0}(N)$. Let $\Delta$ be a fundamental discriminant and let $r \in \mathbb{Z}$ such that $\Delta \equiv r^{2}$ $(\bmod 4 N)$. For any vector $\lambda \in L^{\prime}$ of negative norm, the orthogonal complement $\lambda^{\perp} \subset V(\mathbb{R})$ defines a point $H(\lambda)$ in $\operatorname{Gr}(V) \cong \mathbb{H}$. For $h \in L^{\prime} / L$ and a negative rational number $m \in \mathbb{Z}+\operatorname{sgn}(\Delta) Q(h)$, we consider the twisted Heegner divisor

$$
H_{\Delta, r}(m, h):=\sum_{\lambda \in L_{d \Delta, h r} / \Gamma_{0}(N)} \frac{\chi_{\Delta}(\lambda)}{w(\lambda)} H(\lambda) \in \operatorname{Div}\left(X_{0}(N)\right)_{\mathbb{Q}}
$$

where $d:=4 N m \operatorname{sgn}(\Delta) \in \mathbb{Z}$. Note that $d$ is a discriminant which is congruent to a square modulo $4 N$ and which has the opposite sign as $\Delta$. Here $w(\lambda)$ is the order of the stabilizer of $\lambda$ in $\Gamma_{0}(N)$. We also require the degree zero divisor

$$
y_{\Delta, r}(m, h):=H_{\Delta, r}(m, h)-\operatorname{deg}\left(H_{\Delta, r}(m, h)\right) \cdot \infty .
$$

We have $y_{\Delta, r}(f)=H_{\Delta, r}(f)$ when $\Delta \neq 1$. By the theory of complex multiplication, the divisor $H_{\Delta, r}(m, h)$ is defined over $\mathbb{Q}(\sqrt{\Delta})$.

Recall that $\tilde{\rho}_{L}=\rho_{L}$ for $\Delta>0$, and $\tilde{\rho}_{L}=\bar{\rho}_{L}$ for $\Delta<0$. Suppose that $f \in H_{1 / 2, \tilde{\rho}_{L}}$ is a harmonic weak Maass form of weight $1 / 2$ with representation $\tilde{\rho}_{L}$. We denote the coefficients of $f=f^{+}+f^{-}$by $c^{ \pm}(m, h)$ as in (14.10). 
Using the Fourier coefficients of the principal part of $f$, we finally define the twisted Heegner divisor associated to $f$ by

$$
\begin{aligned}
H_{\Delta, r}(f) & :=\sum_{h \in L^{\prime} / L} \sum_{m<0} c^{+}(m, h) H_{\Delta, r}(m, h) \in \operatorname{Div}\left(X_{0}(N)\right)_{\mathbb{R}}, \\
y_{\Delta, r}(f) & :=\sum_{h \in L^{\prime} / L} \sum_{m<0} c^{+}(m, h) y_{\Delta, r}(m, h) \in \operatorname{Div}\left(X_{0}(N)\right)_{\mathbb{R}} .
\end{aligned}
$$

Notice that $y_{\Delta, r}(f)=H_{\Delta, r}(f)$ when $\Delta \neq 1$.

THEOREM 14.5. Assume the notation and hypotheses above. Let $f \in$ $H_{1 / 2, \tilde{\rho}_{L}}$ be a harmonic Maass form with real coefficients $c^{+}(m, h)$ for all $m \in \mathbb{Q}$ and $h \in L^{\prime} / L$. Moreover, assume that $c^{+}(n, h) \in \mathbb{Z}$ for all $n \leq 0$. Then infinite product

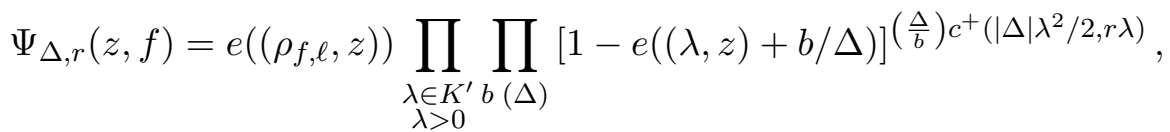

where $\rho_{f, \ell}$ is a certain Weyl vector (see (4.8) of [67]), converges for $y$ sufficiently large and has a meromorphic continuation to all of $\mathbb{H}$ with the following properties.

(i) It is a meromorphic modular form for $\Gamma_{0}(N)$ with a unitary character $\sigma$ which may have infinite order.

(ii) The weight of $\Psi_{\Delta, r}(z, f)$ is $c^{+}(0,0)$ when $\Delta=1$, and is 0 when $\Delta \neq 1$.

(iii) The divisor of $\Psi_{\Delta, r}(z, f)$ on $X_{0}(N)$ is given by $H_{\Delta, r}(f)+C_{\Delta, r}(f)$, where $C_{\Delta, r}(f)$ is supported at cusps.

(iv) The "regularized theta integral" of $f$ defined in (5.5) of [67], satisfies

$$
\Phi_{\Delta, r}(z, f)=\left\{\begin{array}{cl}
-c^{+}(0,0)\left(\log (4 \pi N)+\Gamma^{\prime}(1)\right) & \\
-4 \log \left|\Psi_{\Delta, r}(z, f) y^{c^{+}(0,0) / 2}\right|, & \text { if } \Delta=1, \\
2 \sqrt{\Delta} c(0,0) L\left(1, \chi_{\Delta}\right)-4 \log \left|\Psi_{\Delta, r}(z, f)\right|, & \text { if } \Delta \neq 1 .
\end{array}\right.
$$

Unlike the results in $[\mathbf{4 3}]$, such generalized Borcherds products typically transform with a multiplier system of infinite order under $\Gamma_{0}(N)$. The following criterion is obtained which determines when the multiplier system has finite order.

Theorem 14.6. Suppose that $\Delta \neq 1$. Let $f \in H_{1 / 2, \tilde{\rho}_{L}}$ be a harmonic Maass form with real coefficients $c^{+}(m, h)$ for all $m \in \mathbb{Q}$ and $h \in L^{\prime} / L$. Moreover, assume that $c^{+}(n, h) \in \mathbb{Z}$ for all $n \leq 0$. The following are equivalent.

(i) The character $\sigma$ of the function $\Psi_{\Delta, r}(z, f)$ defined in Theorem 14.5 is of finite order.

(ii) The coefficients $c^{+}\left(|\Delta| \lambda^{2} / 2, r \lambda\right)$ are rational for all $\lambda \in K^{\prime}$. 
REMARK 54. Theorem 14.6 is particularly simple to implement for weakly holomorphic modular forms $f$ (for example, see Lemma 6.5 of [67]). In this case the rationality of all the coefficients is implied by the rationality of the principal part, and so Theorem 14.6 implies that the $\sigma$ for the corresponding generalized Borcherds product has finite order.

ExAmple 14.7 (Twisted modular polynomials). Here we use Theorems 14.5 and 14.6 to deduce the infinite product expansion of twisted modular polynomials found by Zagier (see Section 7 of [212]).

The weight $1 / 2$ harmonic Maass forms in question are the same weakly holomorphc modular forms $f_{d}(z)$ which appear in (14.5). Theorems 14.5 and 14.6 then give a meromorphic modular form $\Psi_{\Delta}\left(z, f_{d}\right):=\Psi_{\Delta, r}\left(z, f_{d}\right)$ of weight 0 for the group $\Gamma=\mathrm{SL}_{2}(\mathbb{Z})$ whose divisor on $X(1)$ is given by

$$
H_{\Delta}(d):=H_{\Delta, r}(d / 4, d / 2)=\sum_{\lambda \in L_{\Delta d} / \Gamma} \frac{\chi_{\Delta}(\lambda)}{w(\lambda)} \cdot H(\lambda) .
$$

Classically, the quotient $L_{\Delta d} / \Gamma$ corresponds to the $\Gamma$-classes of integral binary quadratic forms of discriminant $\Delta d$. Moreover, for sufficiently large $\operatorname{Im}(z)$, we have the product expansion

$$
\Psi_{\Delta}\left(z, f_{d}\right)=\prod_{n=1}^{\infty} \prod_{b(\Delta)}[1-e(n z+b / \Delta)]^{\left(\frac{\Delta}{b}\right) c_{d}\left(\Delta n^{2}\right)} .
$$

From these properties it follows that

$$
\Psi_{\Delta}\left(z, f_{d}\right)=\prod_{\lambda \in L_{\Delta d} / \Gamma}(j(z)-j(H(\lambda)))^{\chi_{\Delta}(\lambda)} .
$$

As an example, let $\Delta:=5$ and $d:=-3$. There are two classes of binary quadratic forms of discriminant -15 , represented by $[1,1,4]$ and $[2,1,2]$, and their corresponding CM points are $\frac{-1+\sqrt{-15}}{2}$ and $\frac{-1+\sqrt{-15}}{4}$. It is well known that the singular moduli of $j(\tau)$ of these points are $-\frac{191025}{2}-\frac{85995}{2} \sqrt{5}$, and $-\frac{191025}{2}+\frac{85995}{2} \sqrt{5}$. The function $f_{-3}$ has the Fourier expansion

$$
f_{-3}=q^{-3}-248 q+26752 q^{4}-85995 q^{5}+1707264 q^{8}-4096248 q^{9}+\ldots
$$

Multiplying out the product over $b$ in (14.20), we obtain the infinite product expansion

$$
\Psi_{5}\left(z, f_{-3}\right)=\frac{j(z)+\frac{191025}{2}+\frac{85995}{2} \sqrt{5}}{j(z)+\frac{191025}{2}-\frac{85995}{2} \sqrt{5}}=\prod_{n=1}^{\infty}\left(\frac{1+\frac{1-\sqrt{5}}{2} q^{n}+q^{2 n}}{1+\frac{1+\sqrt{5}}{2} q^{n}+q^{2 n}}\right)^{c_{-3}\left(5 n^{2}\right)} .
$$

EXAMPLE 14.8 (A generalized Borcherds product for Ramanujan's $\omega(q)$ ). Here we give an example of a generalized Borcherds product arising from 
Ramanujan's mock theta function

$$
\begin{aligned}
\omega(q):= & \sum_{n=0}^{\infty} \frac{q^{2 n^{2}+2 n}}{\left(q ; q^{2}\right)_{n+1}^{2}}=\frac{1}{(1-q)^{2}}+\frac{q^{4}}{(1-q)^{2}\left(1-q^{3}\right)^{2}} \\
& +\frac{q^{12}}{(1-q)^{2}\left(1-q^{3}\right)^{2}\left(1-q^{5}\right)^{2}}+\cdots .
\end{aligned}
$$

Zwegers's work (see Theorem 6.1) completes this $q$-series in a way which allows him to produce a vector valued harmonic Maass form.

Define integers $a(n)$ by

$$
\begin{aligned}
-2 q^{1 / 3} & \left(\omega\left(q^{1 / 2}\right)+\omega\left(-q^{1 / 2}\right)\right) \\
= & : \sum_{n \in \mathbb{Z}+1 / 3} a(n) q^{n}=-4 q^{1 / 3}-12 q^{4 / 3}-24 q^{7 / 3}-40 q^{10 / 3}-\ldots
\end{aligned}
$$

If we let

$$
P(X):=\frac{1+\sqrt{-2} X-X^{2}}{1-\sqrt{-2} X-X^{2}}
$$

then Theorems 14.5 and 14.6 imply that

$$
\Psi(z)=\prod_{n=1}^{\infty} P\left(q^{n}\right)^{\left(\frac{n}{3}\right) a\left(n^{2} / 3\right)}
$$

is a modular function on $\Gamma_{0}(6)$.

Here we work out an expression for $\Psi(z)$. We use the Hauptmodul for $\Gamma_{0}^{*}(6)$, the extension of $\Gamma_{0}(6)$ by all Atkin-Lehner involutions, which is

$$
\begin{aligned}
j_{6}^{*}(z) & =\left(\frac{\eta(z) \eta(2 z)}{\eta(3 z) \eta(6 z)}\right)^{4}+4+3^{4}\left(\frac{\eta(3 z) \eta(6 z)}{\eta(z) \eta(2 z)}\right)^{4} \\
& =q^{-1}+79 q+352 q^{2}+1431 q^{3}+\ldots
\end{aligned}
$$

Let $\alpha_{1}$ and $\alpha_{2}$ be the Heegner points

$$
\alpha_{1}:=\frac{-2+\sqrt{-2}}{6} \text { and } \alpha_{2}:=\frac{2+\sqrt{-2}}{6} .
$$

We have $j_{6}^{*}\left(\alpha_{1}\right)=j_{6}^{*}\left(\alpha_{2}\right)=-10$. Hence $j_{6}^{*}(z)+10$ is a rational function on $X_{0}(6)$ whose divisor consists of the 4 cusps with multiplicity -1 and the points $\alpha_{1}, \alpha_{2}$ with multiplicity 2 . The unique normalized cusp form of weight 4 for $\Gamma_{0}^{*}(6)$ is

$$
\begin{aligned}
\delta(z) & :=\eta(z)^{2} \eta(2 z)^{2} \eta(3 z)^{2} \eta(6 z)^{2} \\
& =q-2 q^{2}-3 q^{3}+4 q^{4}+6 q^{5}+6 q^{6}-16 q^{7}-8 q^{8}+\ldots
\end{aligned}
$$

Using these functions, we find that

$$
\phi(z):=\Psi(z) \cdot\left(j_{6}^{*}(z)+10\right) \delta(z)
$$


is a holomorphic modular form of weight 4 for $\Gamma_{0}(6)$ with divisor $4\left(\alpha_{1}\right)$. Using the classical Eisenstein series, it turns out that

$$
\begin{aligned}
450 \phi(z)= & (3360-1920 \sqrt{-2}) \delta(z)+(1-7 \sqrt{-2}) E_{4}(z)+(4-28 \sqrt{-2}) E_{4}(2 z) \\
& +(89+7 \sqrt{-2}) E_{4}(3 z)+(356+28 \sqrt{-2}) E_{4}(6 z) .
\end{aligned}
$$

Putting this all together, (14.23) becomes

$$
\begin{gathered}
\prod_{n=1}^{\infty}\left(\frac{1+\sqrt{-2} q^{n}-q^{2 n}}{1-\sqrt{-2} q^{n}-q^{2 n}}\right)^{\left(\frac{n}{3}\right) a\left(n^{2} / 3\right)}=\frac{\phi(z)}{\left(j_{6}^{*}(z)+10\right) \delta(z)} \\
=1-8 \sqrt{-2} q-(64-24 \sqrt{-2}) q^{2}+(384+168 \sqrt{-2}) q^{3} \\
\quad+(64-1768 \sqrt{-2}) q^{4}+\cdots .
\end{gathered}
$$

\section{Derivatives and values of modular $L$-functions}

Harmonic Maass forms are very useful for investigating the central values and derivatives of weight 2 modular $L$-functions. Recent works by the author, Bruinier, and Yang involve the interplay between generalized Borcherds products, harmonic Maass forms, and earlier celebrated theorems of Gross and Zagier, Kohnen and Zagier, and Waldspurger.

To explain these results, we first recall some standard definitions. Let

$$
f(z)=\sum_{n=1}^{\infty} a(n) q^{n} \in S_{2 k}^{\text {new }}(M)
$$

be a newform of even integral weight $2 k$ on $\Gamma_{0}(M)$. For $\operatorname{Re}(s) \gg 0$, let

$$
L(f, s)=\sum_{n=1}^{\infty} \frac{a(n)}{n^{s}}
$$

be its $L$-function. Let $D$ denote the fundamental discriminant of a quadratic field, and let $\chi_{D}=\left(\frac{D}{\bullet}\right)$ denote the Kronecker character for the field $\mathbb{Q}(\sqrt{D})$. The $D$-quadratic twist of $f(z)$, denoted $f_{D}(z)$, is given by

$$
f_{D}(z)=\sum_{n=1}^{\infty} \chi_{D}(n) a(n) q^{n},
$$

and for $\operatorname{Re}(s) \gg 0$ its $L$-function is given by

$$
L\left(f, \chi_{D}, s\right)=\sum_{n=1}^{\infty} \frac{\chi_{D}(n) a(n)}{n^{s}} .
$$

These $L$-functions have analytic continuations to $\mathbb{C}$ and satisfy well known functional equations. If we define $\Lambda(f, s)$ by

$$
\Lambda(f, s):=(2 \pi)^{-s} \Gamma(s) M^{s / 2} L(f, s),
$$

then there is an $\epsilon \in\{ \pm 1\}$ for which

$$
\Lambda(f, s)=\epsilon \cdot \Lambda(f, 2 k-s) .
$$


Furthermore, if $D$ is a fundamental discriminant which is coprime to $M$, then

$$
\Lambda\left(f_{D}, s\right)=\epsilon \cdot \chi_{D}(-M) \Lambda\left(f_{D}, 2 k-s\right) .
$$

REMARK 55. The number $\epsilon$ above is referred to as the sign of the functional equation of $L(f, s)$. If $f(z) \in S_{2 k}^{\text {new }}(M)$ is a newform, then

$$
\epsilon=(-1)^{k} \lambda_{M},
$$

where $\lambda_{M}$ is the eigenvalue of $f(z)$ under the Fricke involution $W(M)$. In other words, we have

$$
\left(\left.f\right|_{2 k} W(M)\right)(z)=\lambda_{M} f(z) .
$$

The values $L\left(f, \chi_{D}, k\right)$ are the central critical values of the $L$-functions $L\left(f, \chi_{D}, s\right)$.

Our motivating problem is to describe the behavior of the values $L\left(f, \chi_{D}, k\right)$, as $D$ varies. Notice that if $\chi_{D}(-M) \epsilon=-1$, then $L\left(f, \chi_{D}, k\right)=$ 0 . Therefore at least half of these $L\left(f, \chi_{D}, k\right)$ are trivially zero. As we shall see, the "nontrivial zeros" are much more mysterious.

Much of the interest in central critical values of modular $L$-functions follows from their connection to the Birch and Swinnerton-Dyer. Here we briefly recall important facts and results.

Suppose that $E / \mathbb{Q}$ is an elliptic curve of conductor $N(E)$ (see $[\mathbf{1 9 4}]$ for background on elliptic curves), and let, for $\operatorname{Re}(s) \gg 0$,

$$
L(E, s)=\sum_{n=1}^{\infty} \frac{a_{E}(n)}{n^{s}}
$$

be its Hasse-Weil $L$-function. In particular, if $p \nmid N(E)$ is prime, then we have

$$
N_{E}(p)=p+1-a_{E}(p),
$$

where $N_{E}(p)$ denotes the number of points on the reduction of $E$ modulo $p$.

By the work of Breuil, Conrad, Diamond, Taylor, and Wiles [46, 81, $\mathbf{1 9 8}, \mathbf{2 0 9}$ ], we have the following deep result which confirmed the ShimuraTaniyama Conjecture.

THEOREM 15.1. If $E / \mathbb{Q}$ is an elliptic curve of conductor $N(E)$, then there is a newform $f_{E}(z) \in S_{2}^{\text {new }}(N(E))$ for which

$$
L(E, s)=L\left(f_{E}, s\right) .
$$

For elliptic curves $E / \mathbb{Q}$, Theorem 15.1 implies that $L(E, s)$ has an analytic continuation to $\mathbb{C}$, and so the analytic behavior of $L(E, s)$ at $s=1$ is well defined. The Birch and Swinnerton-Dyer Conjecture (see Appendix C of [194]) gives arithmetic significance to this behavior.

Conjecture. If $E / \mathbb{Q}$ is an elliptic curve, and if $\operatorname{rk}(E)$ is the MordellWeil rank of $E$ over $\mathbb{Q}$, then

$$
\operatorname{ord}_{s=1}(L(E, s))=\operatorname{rk}(E) .
$$


Remark 56. This is the "weak" form of the Birch and Swinnerton-Dyer Conjecture. The strong conjecture gives an arithmetic formula for

$$
\lim _{s \rightarrow 1}\left((s-1)^{-\mathrm{rk}(E)} \cdot L(E, s)\right)
$$

in terms of arithmetic invariants associated to $E$.

Although this conjecture remains open, we have the following strong result which follows from the work of Kolyvagin, Gross, and Zagier [108, 138]. ${ }^{18}$

THEOREM 15.2. If $E / \mathbb{Q}$ is an elliptic curve for which $\operatorname{ord}_{s=1}(L(E, s)) \in$ $\{0,1\}$, then

$$
\operatorname{ord}_{s=1}(L(E, s))=\operatorname{rk}(E) .
$$

Now we consider quadratic twists of elliptic curves and modular forms. Let $E / \mathbb{Q}$ be an elliptic curve given by the Weierstrass equation

$$
E: y^{2}=x^{3}+a x^{2}+b x+c,
$$

where $a, b$ and $c$ are integers. If $D$ is a square-free integer, then let $E(D)$ denote the $D$-quadratic twist of $E$ given by

$$
E(D): y^{2}=x^{3}+a D x^{2}+b D^{2} x+c D^{3} .
$$

The curves $E$ and $E(D)$ are isomorphic over $\mathbb{Q}(\sqrt{D})$.

Suppose that $E / \mathbb{Q}$ is an elliptic curve, and that

$$
f_{E}(z)=\sum_{n=1}^{\infty} a_{E}(n) q^{n} \in S_{2}^{\text {new }}(N(E))
$$

is the weight two newform associated to $E$ by Theorem 15.1. Similarly, if $D$ is a fundamental discriminant, then let

$$
f_{E(D)}(z)=\sum_{n=1}^{\infty} a_{E(D)}(n) q^{n}
$$

be the newform associated to $E(D)$. If $p \nmid D N(E)$ is prime, then it is simple to check that

$$
a_{E(D)}(p)=\left(\frac{D}{p}\right) a_{E}(p) .
$$

Consequently, the newform $f_{E(D)}(z)$ is the unique newform whose Hecke eigenvalues, for primes $p \nmid D N(E)$, equal those of the quadratic twist $f_{E}$ by $\chi_{D}$.

In view of the Birch and Swinnerton-Dyer Conjecture, and Theorem 15.2, we are compelled to study central values and derivatives of weight 2 modular $L$-functions. In this direction we have the celebrated works of Kohnen, Zagier and Waldspurger, and also the work of Gross and Zagier [108].

\footnotetext{
${ }^{18}$ Coates and Wiles $[\mathbf{7 9}]$ earlier proved that $L(E, 1) \neq 0 \Longrightarrow \operatorname{rk}(E)=0$ for elliptic curves $E / \mathbb{Q}$ with complex multiplication.
} 
It turns out that the Fourier coefficients of half-integral weight cusp forms often interpolate the "square-roots" of the central critical values of the $L$-functions of the quadratic twists of even weight newforms.

Here we make this precise. First suppose that $N$ is odd and square-free, and suppose further that

$$
g(z)=\sum_{n=1}^{\infty} b(n) q^{n} \in S_{k+\frac{1}{2}}^{\text {new }}(4 N)
$$

is a Kohnen newform. ${ }^{19}$ There is a unique newform, say $f(z) \in S_{2 k}^{\text {new }}(N)$, associated to $g(z)$ under Shimura's correspondence. The coefficients of $g(z)$ determine the central critical values of many of the quadratic twists $L\left(f, \chi_{D}, s\right)$. Let $\nu(N)$ denote the number of distinct prime divisors of $N$, and let $\langle f, f\rangle$ (resp. $\langle g, g\rangle$ ) denote the Petersson inner product on $S_{2 k}(N)$ (resp. $\left.S_{k+\frac{1}{2}}(4 N)\right)$. Generalizing a result in [137], Kohnen proved the following important theorem in $[\mathbf{1 3 6}]$.

TheOREM 15.3. Assume the notation in the preceding discussion. If $\ell \mid N$ is prime, then let $\lambda_{\ell} \in\{ \pm 1\}$ be the eigenvalue of the Atkin-Lehner involution

$$
\left(\left.f\right|_{2 k} W\left(Q_{\ell}\right)\right)(z)=\lambda_{\ell} f(z) .
$$

If $(-1)^{k} D>0$ and $D$ has the property that $\left(\frac{D}{\ell}\right)=\lambda_{\ell}$ for each prime $\ell \mid N$, then

$$
L\left(f, \chi_{D}, k\right)=\frac{\langle f, f\rangle \cdot \pi^{k}}{2^{\nu(N)}(k-1) !|D|^{k-\frac{1}{2}}\langle g, g\rangle} \cdot|b(|D|)|^{2} .
$$

For all other fundamental discriminants $D$ with $(-1)^{k} D>0$ we have $b(|D|)=0$.

ExAMPLE 15.4. If $f(z)=\Delta(z) \in S_{12}$, then it turns out that the associated Kohnen newform in $S_{13 / 2}^{\text {new }}(4)$ is

$$
\begin{aligned}
g(z) & =\sum_{n=1}^{\infty} b(n) q^{n}=\frac{E_{4}(4 z) \cdot D\left(\Theta_{0}(z)\right)}{2}-\frac{D\left(E_{4}(4 z)\right) \cdot \Theta_{0}(z)}{16} \\
& =q-56 q^{4}+120 q^{5}-\ldots
\end{aligned}
$$

Again, here $D$ denotes the differential operator $D:=q \frac{d}{d q}$. For positive fundamental discriminants $D$, we then have that

$$
L\left(\Delta, \chi_{D}, 6\right)=\frac{\langle\Delta, \Delta\rangle \cdot \pi^{6}}{120 D^{\frac{11}{2}}\langle g, g\rangle} \cdot b(D)^{2} .
$$

\footnotetext{
$[136]$.

${ }^{19}$ These are the half-integral weight cuspidal Hecke eigenforms in Kohnen's paper
} 
Waldspurger proved a similar theorem which holds in greater generality. For every fundamental discriminant $D$, define $D_{0}$ by

$$
D_{0}:= \begin{cases}|D| & \text { if } D \text { is odd, } \\ |D| / 4 & \text { if } D \text { is even. }\end{cases}
$$

The following is a convenient reformulation of Waldspurger's theorem [205].

THEOREM 15.5. If $f(z)=\sum_{n=1}^{\infty} a(n) q^{n} \in S_{2 k}^{\text {new }}(M)$ is an even weight newform and $\delta \in\{ \pm 1\}$ is the sign of the functional equation of $L(f, s)$, then there is a positive integer $N$ with $M \mid N$, a Dirichlet character $\chi$ modulo $4 N$, a nonzero complex number $\Omega_{f}$, and a nonzero half-integral weight Hecke eigenform

$$
g_{f}(z)=\sum_{n=1}^{\infty} b_{f}(n) q^{n} \in S_{k+\frac{1}{2}}(4 N, \chi)
$$

with the property that there are arithmetic progressions of fundamental discriminants $D$ coprime to $4 N$ for which $\delta D>0$ and

$$
L\left(f, \chi_{D}, k\right)=\epsilon_{D} \cdot \frac{b_{f}\left(D_{0}\right)^{2}}{D_{0}^{k-\frac{1}{2}}} \cdot \Omega_{f},
$$

where $\epsilon_{D}$ is algebraic. For all other $D$ with $\delta D>0$, we have $b_{f}\left(D_{0}\right)=0$. Moreover, the coefficients $a(n), b_{f}(n)$ and the values of $\chi$ are in $\mathcal{O}_{K}$, the ring of integers of some fixed number field $K$.

15.1. Extension of the Kohnen-Waldspurger theorem. The author and Bruinier [67] have generalized this theorem of Waldspurger and Kohnen to prove that the Fourier coefficients of weight $1 / 2$ harmonic Maass forms encode the vanishing and nonvanishing of both the central values and derivatives of quadratic twists of weight 2 modular $L$-functions.

Here we describe a special case of the main result of $[67]$.

THEOREM 15.6. Suppose that

$$
G(z)=\sum_{n=1}^{\infty} B_{G}(n) q^{n} \in S_{2}(p)
$$

is a weight 2 newform with prime level $p$. In addition, suppose that the sign of the functional equation of $L(G, s)$ is $\epsilon(G)=-1$. Then there is a weight 1/2 harmonic Maass form $f(z)$ on $\Gamma_{0}(4 p)$, say

$$
f(z)=\sum_{n \gg-\infty} c_{g}^{+}(n) q^{n}+\sum_{n<0} c_{g}^{-}(n) \Gamma(1 / 2 ; 4 \pi|n| y) q^{n},
$$

which satisfies the following:

(1) If $\Delta<0$ is a fundamental discriminant for which $\left(\frac{\Delta}{p}\right)=1$, then

$$
L\left(G, \chi_{\Delta}, 1\right)=\alpha_{G} \cdot \sqrt{|\Delta|} \cdot c_{g}^{-}(\Delta)^{2},
$$

where $\alpha_{G}$ is an explicit non-zero constant. 
(2) If $\Delta>0$ is a fundamental discriminant for which $\left(\frac{\Delta}{p}\right)=1$, then $L^{\prime}\left(G, \chi_{\Delta}, 1\right)=0$ if and only if $c_{g}^{+}(\Delta)$ is algebraic.

REMARK 57. Theorem 15.6 is a special case of the general result which holds for all levels, and any arbitrary sign. Notice that the result concerns all weight 2 newforms, not just those which correspond to modular elliptic curves.

REMARK 58. The Maass form $f(z)$ in Theorem 15.6 is unique up to the addition of a weight $1 / 2$ weakly holomorphic modular form with coefficients in $F_{G}$, the number field obtained by adjoining the coefficients of $G(z)$ to $\mathbb{Q}$. In view of this ambiguity, it would be very interesting to pin down a choice of $f(z)$ which in turn gives a precise formula relating $L^{\prime}\left(G, \chi_{\Delta}, 1\right)$ to $c_{g}^{+}(\Delta)$ in Theorem $15.6(2)$.

Theorem 15.6 is obtained by combining the Gross-Zagier formula with Borcherds products arising from harmonic Maass forms and general transcendence theorems for canonical differentials of the third kind on modular curves. Here we give a brief sketch of the proof of this theorem.

Sketch of the Proof of Theorem 15.6. By Kohnen's theory, there is a half-integral weight newform

$$
g(z)=\sum_{n=1}^{\infty} b_{g}(n) q^{n} \in S_{\frac{3}{2}}^{+}(4 p),
$$

unique up to a multiplicative constant, which lifts to $G$ under the Shimura correspondence. We can (and do) choose $g$ so that its coefficients are in $F_{G}$, the totally real number field obtained by adjoining the Fourier coefficients of $G$ to $\mathbb{Q}$. We prove that there is a weight $1 / 2$ harmonic Maass form on $\Gamma_{0}(4 p)$ in the plus space, say

$$
f_{g}(z)=\sum_{n \gg-\infty} c_{g}^{+}(n) q^{n}+\sum_{n<0} c_{g}^{-}(n) \Gamma(1 / 2,4 \pi|n| y) q^{n},
$$

whose principal part $P_{f_{g}}$ has coefficients in $F_{G}$, which also enjoys the property that $\xi_{\frac{1}{2}}\left(f_{g}\right)=\|g\|^{-2} g$, where $\|g\|$ denotes the usual Petersson norm.

By Lemma 7.4, if $n>0$, then

$$
b_{g}(n)=-4 \sqrt{\pi n}\|g\|^{2} \cdot c_{g}^{-}(-n) .
$$

Theorem 15.6 (1) now follows from Theorem 15.3.

The proof of Theorem 15.6 (2) is more difficult, and it involves a detailed study of Heegner divisors. We establish that the algebraicity of the coefficients $c_{g}^{+}(\Delta)$ is dictated by the vanishing of certain twisted Heegner divisors in the Jacobian of $X_{0}(p)$, which when combined with the work of Gross and Zagier [108] and Scholl and Waldschmidt, then implies Theorem 15.6 (2).

To make this precise, let $d<0$ and $\Delta>0$ be fundamental discriminants which are both squares modulo $p$. Let $\mathcal{Q}_{d, p}$ be the set of discriminant $d=$ $b^{2}-4 a c$ integral binary quadratic forms $a X^{2}+b X Y+c Y^{2}$ with the property 
K. ONO

that $p \mid a$. For these pairs of discriminants, we define the twisted Heegner divisor $H_{\Delta}(d)$ by

$$
H_{\Delta}(d):=\sum_{Q \in \mathcal{Q}_{\Delta d, p} / \Gamma_{0}(p)} \chi_{\Delta}(Q) \cdot \frac{\tau_{Q}}{w_{Q}},
$$

where $\chi_{\Delta}$ denotes the generalized genus character corresponding to the decomposition $\Delta \cdot d$ as in [107], $\tau_{Q}$ is the unique root of $Q(x, 1)$ in $\mathbb{H}$ as before, and $w_{Q}$ denotes the order of the stabilizer of $Q$ in $\Gamma_{0}(p)$. Then $H_{\Delta}(d)$ is a divisor on $X_{0}(p)$ defined over $\mathbb{Q}(\sqrt{\Delta})$. We use these twisted Heegner divisors to define the degree 0 divisor

$$
y_{\Delta}(d):=H_{\Delta}(d)-\operatorname{deg}\left(H_{\Delta}(d)\right) \cdot \infty .
$$

Finally, we associate a divisor to $f_{g}$ by letting

$$
y_{\Delta}\left(f_{g}\right):=\sum_{n<0} c_{g}^{+}(n) y_{\Delta}(n) \in \operatorname{Div}^{0}\left(X_{0}(p)\right) \otimes F_{G} .
$$

Let $J$ be the Jacobian of $X_{0}(p)$, and let $J(F)$ denote the points of $J$ over a number field $F$. The Hecke algebra acts on $J(F) \otimes \mathbb{C}$, which by the Mordell-Weil Theorem is a finite dimensional vector space. We show that the point corresponding to $y_{\Delta}\left(f_{g}\right)$ in $J(\mathbb{Q}(\sqrt{\Delta})) \otimes \mathbb{C}$ is in its $G$-isotypical component. Moreover, we show that the following are equivalent:

(i) The Heegner divisor $y_{\Delta}\left(f_{g}\right)$ vanishes in $J(\mathbb{Q}(\sqrt{\Delta})) \otimes \mathbb{C}$.

(ii) The coefficient $c_{g}^{+}(\Delta)$ is algebraic.

(iii) The coefficient $c_{g}^{+}(\Delta)$ is contained in $F_{G}$.

To obtain these results, we explicitly construct modular functions with prescribed Heegner divisors using results related to Theorems 14.5 and 14.6. By work of Scholl and Waldschmidt, the vanishing of the points corresponding to these divisors is equivalent to the algebraicity of the Fourier expansions of these modular functions. Using the Hecke algebra and the explicit formulas provided by the generalized Borcherds product, we then find that this algebraicity is dictated by single coefficients of the form $c_{g}^{+}(\Delta)$.

We then obtain the following generalization of the well known GrossKohnen-Zagier theorem [107]. Namely, we show that

$$
\sum_{n>0} y_{\Delta}^{G}(-n) q^{n}=g(\tau) \otimes y_{\Delta}\left(f_{g}\right) \in S_{\frac{3}{2}}^{+}(4 p) \otimes J(\mathbb{Q}(\sqrt{\Delta})),
$$

where $y_{\Delta}^{G}(-n)$ denotes the projection of $y_{\Delta}(-n)$ onto its $G$-isotypical component. This result, when combined with the Gross-Zagier theorem [108], gives the conclusion that the Heegner divisor $y_{\Delta}\left(f_{g}\right)$ vanishes in $J(\mathbb{Q}(\sqrt{\Delta})) \otimes \mathbb{C}$ if and only if $L^{\prime}\left(G, \chi_{\Delta}, 1\right)=0$, thereby proving Theorem $15.6(2)$. 
15.2. Recent work of Bruinier and Yang. Building on the results of the last subsection, it is natural to seek exact formulas for derivatives of $L$ functions using techniques arising from the theory of harmonic Maass forms. Theorem 15.6 makes the connection between derivatives and coefficients of harmonic Maass forms, but it does not give exact formulas. This problem remains open. Nevertheless, the question of fundamental interest really should be: Can one directly obtain exact arithmetic formulas (perhaps in terms of heights) for certain derivatives of $L$-functions?

To this end, Bruinier and Yang [70] have recently proven some striking theorems about Faltings heights of CM cycles and derivatives of $L$-functions. In some important cases they are able to explicitly evaluate derivatives of certain Rankin-type $L$-functions in terms of Faltings heights. Their work depends critically on Borcherds lifts and the theory of harmonic Maass forms.

It is impossible to survey their work in any detail here. Instead, we give the flavor of their exciting program. Loosely speaking, they use the principal parts of suitable harmonic Maass forms $f$ to construct Heegner divisors on certain Shimura varieties. This depends on earlier work of Kudla, recent work on Greens functions, and Borcherds lifts. For the cusp forms $\xi(f)$, they investigate a certain "Rankin-type" $L$-function, and they conjecture an exact formula relating its derivative at $s=0$ to the Faltings height pairing of a Heegner divisor with a CM cycle. The conjectured formula is of the form

$$
\langle\hat{\mathcal{Z}}(f), \mathcal{Z}(U)\rangle_{F a l}=\kappa \cdot L^{\prime}(\xi(f), U, 0)
$$

where $\kappa$ is explicit.

They have obtained several deep results in the direction of this conjecture. Here we highlight one implication of their work in the classical setting of the original formula of Gross and Zagier. Suppose that $G$ is a normalized newform of weight 2 for $\Gamma_{0}(N)$ whose Hecke $L$-function $L(G, s)$ satisfies an odd functional equation. Then there is a weight $3 / 2$ cusp form $g$, which corresponds to $G$ under the Shimura correspondence, and it turns out that the "Rankin-type" $L$-function is proportional to $L(G, s+1)$. Their conjecture therefore then implies a Faltings height pairing formula for $L^{\prime}(G, 1)$.

As in the work of the author and Bruinier [67] (see Theorem 5.1), there is a weight $1 / 2$ harmonic Maass form $f$ with vanishing constant term such that $\xi(f)=\|g\|^{-2} g$, with the additional property that the coefficients of its principal part are in the number field generated by the eigenvalues of $G$. In this case the Heegner divisor $Z(f)$ defines an explicit point in the Jacobian of $X_{0}(N)$, which lies in the $G$ isotypical component (see the sketch of the proof of Theorem 15.6). In this setting, they prove the following comprehensive theorem. 
Theorem 15.7 (Bruinier and Yang [70]). Assuming the notation and hypotheses above, we have that the Neron-Tate height of $Z(f)$ is given by

$$
\langle Z(f), Z(f)\rangle_{N T}=\frac{2 \sqrt{N}}{\pi\|g\|^{2}} L^{\prime}(G, 1) .
$$

This beautiful theorem directly gives an arithmetic formula for the central derivative of weight 2 modular $L$-functions with odd sign. It is natural to ask how Theorem 15.7 relates to the classical Gross-Zagier formula. The short answer is that it implies it.

To see this, suppose that $E$ is an elliptic curve over $\mathbb{Q}$. Assume that its $L$-function $L(E, s)$ has an odd functional equation so that the central critical value $L(E, 1)$ vanishes. Let $N=N(E)$ be the conductor of $E$, and let $X_{0}(N)$ be the moduli space of cyclic isogenies of degree $N$ of generalized elliptic curves. Let $K$ be an imaginary quadratic field such that $N$ is the norm of an integral ideal of $K$, and write $D$ for the discriminant of $K$. We may consider the divisor $Z(D)$ on $X_{0}(N)$ given by elliptic curves with complex multiplication by the maximal order of $K$. By the theory of complex multiplication, this divisor is defined over $K$, and its degree $h$ is given by the class number of $K$. Hence the divisor $y(D)=\operatorname{tr}_{K / \mathbb{Q}}(Z(D)-h \cdot(\infty))$ has degree zero and is defined over $\mathbb{Q}$. By the modularity of elliptic curves, we obtain a rational point $y^{E}(D)$ on $E$ using a modular parametrization $X_{0}(N) \rightarrow E$. Arguing a little further (so as to produce $L\left(E, \chi_{D}, 1\right)$ ), one can show that Theorem 15.7 then implies the Gross-Zagier theorem as stated below.

TheOREM 15.8 (Gross-Zagier [108]). Assume the notation and hypotheses above. Then the canonical height of $y^{E}(D)$, denoted $\left\langle y^{E}(D), y^{E}(D)\right\rangle_{N T}$, satisfies

$$
\left\langle y^{E}(D), y^{E}(D)\right\rangle_{N T}=C \sqrt{|D|} L^{\prime}(E, 1) L\left(E, \chi_{D}, 1\right) .
$$

Here $C$ is an explicit non-zero constant which is independent of $K$, and $\chi_{D}$ is the Kronecker character for $K / \mathbb{Q}$.

To place Theorem 15.7 in proper context, we stress that the work of Gross and Zagier has inspired an enormous body of further research on height pairings of algebraic cycles on Shimura varieties. For instance, Zhang considered heights of Heegner type cycles on Kuga-Sato fiber varieties over modular curves in [214], and the heights of Heegner points on compact Shimura curves over totally real fields in [215]. Gross and Keating discovered a connection between arithmetic intersection numbers of Hecke correspondences on the product of two copies of the modular curve $X(1)$ over $\mathbb{Z}$ and the coefficients of the derivative of the Siegel-Eisenstein series of genus three and weight two [106]. This has inspired the extensive program of Kudla, Rapoport and Yang which relates Arakelov intersection numbers on Shimura varieties of orthogonal type to derivatives of Siegel-Eisenstein series and modular $L$-functions (for example, see [140], [141], [142]). 
In all of these works, the connection between a height pairing and the derivative of an automorphic $L$-function arises in an indirect way. The idea has been to identify the local height pairings in the Fourier coefficients of a suitable integral kernel function (often given by an Eisenstein series), which takes an automorphic form $\phi$ to the special value of the derivative of an $L$-function associated to $\phi$.

This recent work of Bruinier and Yang gives a new approach for obtaining identities between certain height pairings on Shimura varieties of orthogonal type and derivatives of automorphic $L$-functions. As described above, it is based on the Borcherds lift [44] and its generalization in $[\mathbf{6 2}, \mathbf{6 3}, \mathbf{6 7}]$. Their approach directly gives formulas for the Faltings height pairing of arithmetic Heegner divisors and CM cycles.

\section{Epilogue}

Although the mock theta functions are humble in origin, they have earned a distinguished role in the legend of Ramanujan. Andrews and Berndt confirm this in their article "Your hit parade: the top ten most fascinating formulas in Ramanujan's lost notebook" [22]. In their amusing informal poll, Ramanujan's work on Dyson's ranks ${ }^{20}$ and the mock theta functions rank first and second! Based on the mathematics born out of these works, as described here, it is a safe bet that ranks and mock theta functions will continue to hold these top spots into the foreseeable future. They certainly have the author's vote!

It is appropriate to end this paper with a few words about the title: "Unearthing the visions of a master: harmonic Maass forms and number theory". Although Ramanujan's last works provided the first examples of such forms, his untimely death and the enigmatic nature of his writings resulted in a great mystery. We will never know how he came up with the mock theta functions. We certainly cannot pretend to know what he fully intended to do with them. However, it is clear that he understood that the mock theta functions would go on to play important roles in number theory, his "visions". The author likes to think that this paper contains some of these visions.

\section{Acknowledgements}

The author thanks George E. Andrews, Dick Askey, Bruce B. Berndt, Matt Boylan, Kathrin Bringmann, Jan Bruinier, Amanda Folsom, Pavel Guerzhoy, Paul Jenkins, Jeremy Lovejoy, Maria Monks, Rob Rhoades, Heather Swan-Rosenthal, Frank Thorne, Tonghai Yang and Luanlei Zhao for their many helpful comments. Without this assistance the author would have been unable to write this paper.

\footnotetext{
${ }^{20}$ Although Dyson defined the notion of a partition rank in 1944 , it is clear that Ramanujan understood the notion in 1920 because of certain identities he recorded in the "Lost Notebook".
} 


\section{References}

[1] M. Abramowitz and I. Stegun, Pocketbook of mathematical functions, Verlag Harri Deutsch (1984).

[2] S. Ahlgren, Distribution of the partition function modulo composite integers $M$, Math. Ann. 318 (2000), no. 4, pages 795-803.

[3] S. Ahlgren and K. Ono, Congruence properties for the partition function, Proc. Natl. Acad. Sci. USA 98 (2001), no. 23, pages 12882-12884.

[4] S. Ahlgren and K. Ono, Addition and counting: the arithmetic of partitions, Notices Amer. Math. Soc. 48 (2001), no. 9, pages 978-984.

[5] S. Ahlgren and K. Ono, Congruences and conjectures for the partition function, $q$-series with applications to combinatorics, number theory, and physics (Urbana, IL, 2000), Contemp. Math., 291, Amer. Math. Soc., Providence, RI, 2001, pages $1-10$.

[6] P. V. Seshu Aiyar and R. Ramachandra Rao, Srinivasa Ramanujan (1887-1920), Collected papers of Ramanujan (Ed. G. H. Hardy, et. al.), Cambridge Univ. Press, Cambrdige, 1927, pages xi-xx.

[7] G. E. Andrews, On the theorems of Watson and Dragonette for Ramanujan's mock theta functions, Amer. J. Math. 88 No. 2 (1966), pages 454-490.

[8] G. E. Andrews, On the general Rogers-Ramanujan theorem, Amer. Math. Soc., Providence, 1974.

[9] G. E. Andrews, An introduction to Ramanujan's lost notebook, Amer. Math. Monthly 86 (1979), pages 89-108.

[10] G. E. Andrews, Hecke modular forms and the Kac-Peterson identities, Trans. Amer. Math. Soc. 283 (1984), pages 451-458.

[11] G. E. Andrews, Combinatorics and Ramanujan's "lost" notebook, Surveys in combinatorics 1985 (Glasgow, 1985), London Math. Soc. Lecture Note Ser., 103, Cambridge Univ. Press, Cambridge, 1985, pages 1-23.

[12] G. E. Andrews, The fifth and seventh order mock theta functions, Trans. Amer. Math. Soc. 293 (1986), no. 1, pages 113-134.

[13] G. E. Andrews, q-series: Their development and application in analysis, number theory, combinatorics physics, and computer algebra, CBMS No. 66, Amer. Math. Soc., Providence, RI, 1986.

[14] G. E. Andrews, Ramanujan's fifth order mock theta functions as constant terms, Ramanujan revisited (Urbana-Champaign, Ill., 1987), Academic Press, Boston, MA, 1988, pages 47-56.

[15] G. E. Andrews, Mock theta functions, Theta functions-Bowdoin 1987, Part 2 (Brunswick, ME, 1987), pages 283-298, Proc. Sympos. Pure Math., 49, Part 2, Amer. Math. Soc., Providence, RI, 1989.

[16] G. E. Andrews, The theory of partitions, (Reprint of the 1976 original), Cambridge Univ. Press, Cambridge, 1998.

[17] G. E. Andrews, Partitions: At the interface of q-series and modular forms, Rankin Memorial Issues, Ramanujan J. 7 (2003), pages 385-400.

[18] G. E. Andrews, Partitions with short sequences and mock theta functions, Proc. Natl. Acad. Sci. USA, 102 No. 13 (2005), pages 4666-4671.

[19] G. E. Andrews, Partitions, Durfee symbols, and the Atkin-Garvan moments of ranks, Invent. Math. 169 (2007), pages 37-73.

[20] G. E. Andrews, R. Askey and R. Roy, Special functions, Cambridge Univ. Press, Cambridge, 1999.

[21] G. E. Andrews and B. C. Berndt, Ramanujan's lost notebook. Part I, Springer, New York, 2005. 
[22] G. E. Andrews and B. C. Berndt, Your hit parade: the top ten most fascinating formulas in Ramanujan's Lost notebook, Not. Amer. Math. Soc. 55 (2008), pages $18-30$.

[23] G. E. Andrews, F. Dyson, and D. Hickerson, Partitions and indefinite quadratic forms, Invent. Math. 91 No. 3 (1988), pages 391-407.

[24] G. E. Andrews and K. Eriksson, Integer partitions, Cambridge Univ. Press, Cambridge, 2004.

[25] G. E. Andrews and F. Garvan, Dyson's crank of a partition, Bull. Amer. Math. Soc. (N.S.) 18 No. 2 (1988), pages 167-171.

[26] G. E. Andrews and F. Garvan, Ramanujan's "lost" notebook. VI. The mock theta conjectures, Adv. in Math. 73 (1989), no. 2, pages 242-255.

[27] G. E. Andrews and D. Hickerson, Ramanujan's "lost" notebook. VII. The sixth order mock theta functions, Adv. Math. 89 (1991), no. 1, pages 60-105.

[28] A. O. L. Atkin, Proof of a conjecture of Ramanujan, Glasgow Math. J. 8, (1967) pages $14-32$.

[29] A. O. L. Atkin, Multiplicative congruence properties and density problems for $p(n)$, Proc. London Math. Soc. (3) 18 (1968), pages 563-576.

[30] A. O. L. Atkin and F. Garvan, Relations between the ranks and cranks of partitions, Ramanujan J. 7 (2003), pages 343-366.

[31] A. O. L. Atkin and H. P. F. Swinnerton-Dyer, Some properties of partitions, Proc. London Math. Soc. 66 No. 4 (1954), pages 84-106.

[32] B. C. Berndt, Ramanujan's notebooks. Part I, Springer-Verlag, New York, 1985.

[33] B. C. Berndt, Ramanujan's notebooks. Part II, Springer-Verlag, New York, 1989.

[34] B. C. Berndt, Ramanujan's notebooks. Part III, Springer-Verlag, New York, 1991.

[35] B. C. Berndt, Ramanujan's notebooks. Part IV, Springer-Verlag, New York, 1994.

[36] B. C. Berndt, Ramanujan's notebooks. Part V, Springer-Verlag, New York, 1998.

[37] B. C. Berndt, Number theory in the spirit of Ramanujan, Student Math. Library, 34, Amer. Math. Soc., Providence, RI, 2006.

[38] B. C. Berndt and K. Ono, Ramanujan's unpublished manuscript on the partition and tau functions with proofs and commentary, The Andrews Festschrift (Maratea, 1998). Sem. Lothar. Combin. 42 (1999), Art. B42c, 63 pp. (electronic).

[39] B. C. Berndt and R. A. Rankin, Ramanujan: letters and commentary, History of Mathematics, 9. Amer. Math. Soc., Providence, RI, 1995.

[40] B. C. Berndt and R. A. Rankin, Ramanujan: essays and surveys, History of Mathematics, 22, Amer. Math. Soc., Providence, RI, 2001.

[41] B. C. Berndt and C. A. Reddi, Two exams taken by Ramanujan in India, Amer. Math. Monthly, 111 (2004), pages 330-339.

[42] R. E. Borcherds, Automorphic forms on $\mathrm{O}_{s+2,2}(\mathbb{R})^{+}$and generalized Kac-Moody algebras, Proceedings of the International Congress of Mathematicians, Vol. 1, 2 (Zürich, 1994), pages 744-752, Birkhäuser, Basel, 1995.

[43] R. E. Borcherds, Automorphic forms on $O_{s+2,2}(\mathbb{R})$ and infinite products, Invent. Math. 120 (1995), pages 161-213.

[44] R. E. Borcherds, Automorphic forms with singularities on Grassmannians, Invent. Math. 132 (1998), 491-562.

[45] M. Boylan, Arithmetic properties of certain level one mock modular forms, Int. J. Number Th., accepted for publication.

[46] C. Breuil, B. Conrad, F. Diamond, and R. Taylor, On the modularity of elliptic curves over $\mathbb{Q}$; wild 3-adic exercises, J. Amer. Math. Soc. 14 (2001), 843-939.

[47] K. Bringmann, On certain congruences for Dyson's ranks, Int. J. Numb. Th., accepted for publication.

[48] K. Bringmann, On the explicit construction of higher deformations of partition statistics, Duke J. Math., 144 (2008), pages 195-233. 
[49] K. Bringmann, A. Folsom, and K. Ono, q-series and weight 3/2 Maass forms, Compositio Math. 145 (2009), pages 541-552.

[50] K. Bringmann, F. Garvan, and K. Mahlburg, Partition statistics and quasiweak Maass forms, Int. Math. Res. Notices, (2008), rmn. 124.

[51] K. Bringmann and J. Lovejoy, Overpartitions and class numbers of binary quadratic forms, Proc. Natl. Acad. Sci., USA, 106 (2009), pages 5513-5516.

[52] K. Bringmann, J. Lovejoy, and R. Osburn, Automorphic properties of generating functions for generalized rank moments and Durfee symbols, Int. Math. Res. Notices, accepted for publication.

[53] K. Bringmann and K. Ono, The $f(q)$ mock theta function conjecture and partition ranks, Invent. Math. 165 (2006), pages 243-266.

[54] K. Bringmann and K. Ono, Arithmetic properties of coefficients of half-integral weight Maass-Poincaré series, Math. Ann., 337 (2007), pages 591-612.

[55] K. Bringmann and K. Ono, Dyson's ranks and Maass forms, Ann. of Math., accepted for publication.

[56] K. Bringmann and K. Ono, Lifting cusp forms to Maass forms with an application to partitions, Proc. Natl. Acad. Sci., USA 104, No. 10 (2007), pages 3725-3731.

[57] K. Bringmann, K. Ono, and R. Rhoades, Eulerian series as modular forms, J. Amer. Math. Soc., 21 (2008), pages 1085-1104.

[58] K. Bringmann, K. Ono, and J. Rouse, Traces of singular moduli on Hilbert modular surfaces, Int. Math. Res. Notices, no. 47 (2005), pages 2891-2912.

[59] K. Bringmann and K. Ono, Coefficients of harmonic weak Maass forms, Proc. Conference on Partitions, $q$-series, and modular forms (Univ. Florida 2008), accepted for publication.

[60] K. Bringmann and S. Zwegers, Rank-crank type PDE's and non-holomorphic Jacobi forms, Math. Res. Lettrs., accepted for publication.

[61] J. H. Bruinier, Borcherds products and Chern classes of Hirzebruch-Zagier divisors, Invent. Math. 138 (1999), pages 51-83.

[62] J. H. Bruinier, Borcherds products on $\mathrm{O}(2, l)$ and Chern classes of Heegner divisors, Springer Lect. Notes Math. 1780, Springer-Verlag (2002).

[63] J. H. Bruinier and J. Funke, On Two Geometric Theta Lifts, Duke Math. Journal 125 (2004), pages 45-90.

[64] J. H. Bruinier and J. Funke, Traces of CM-values of modular functions, J. Reine Angew. Math. 594 (2006), pages 1-33.

[65] J. H. Bruinier, P. Jenkins, and K. Ono, Hilbert class polynomials and traces of singular moduli, Math. Ann. 334 (2006), pages 373-393.

[66] J. H. Bruinier, W. Kohnen, and K. Ono, The arithmetic of the values of modular functions and the divisors of modular forms, Compositio Math. 140 (2004), pages $552-566$.

[67] J. H. Bruinier and K. Ono, Heegner divisors, L-functions and harmonic weak Maass forms, Annals of Math., accepted for publication.

[68] J. H. Bruinier, K. Ono, and R. Rhoades, Differential operators and harmonic weak Maass forms, Math. Ann. 342 (2008), pages 673-693.

[69] J. H. Bruinier and F. Strömberg, Computations of harmonic weak Maass forms, preprint.

[70] J. H. Bruinier and T. Yang, Faltings heights of CM cycles and derivatives of L-functions, Invent. Math., accepted for publication.

[71] D. Bump, Automorphic forms and representations, Cambridge Univ. Press, Cambridge, 1998.

[72] Y.-S. Choi, Tenth order mock theta functions in Ramanujan's lost notebook, Invent. Math. 136 No. 3 (1999), pages 497-569.

[73] Y.-S. Choi, Tenth order mock theta functions in Ramanujan's lost notebook. II, Adv. Math. 156 (2000), no. 2, pages 180-285. 
[74] Y.-S. Choi, Tenth order mock theta functions in Ramanujan's lost notebook. III, Proc. Lond. Math. Soc. (3) 94 (2007), no. 1, pages 26-52.

[75] Y.-S. Choi, Tenth order mock theta functions in Ramanujan's lost notebook. IV, Trans. Amer. Math. Soc. 354 (2002), no. 2, pages 705-733.

[76] Y.-S. Choi, Identities for Ramanujan's sixth-order mock theta functions, Q. J. Math. 53 (2002), no. 2, pages 147-159.

[77] D. Choi, D. Jeon, S.-Y. Kang, and C. Kim, Traces of singular moduli of arbitrary level modular functions, Int. Math. Res. Notices (2007), No. 22.

[78] D. Choi, D. Jeon, S.-Y. Kang, and C. Kim, Exact formulas for traces of singular moduli of higher level modular functions, J. Number Th. 128 (2008), pages $700-707$.

[79] J. Coates and A. Wiles, On the conjecture of Birch and Swinnerton-Dyer, Invent. Math. 39 (1977), 223-251.

[80] H. Cohen, q-identities for Maass waveforms, Invent. Math. 91 No. 3 (1988), pages 409-422.

[81] B. Conrad, F. Diamond, and R. Taylor, Modularity of certain potentially BarsottiTate Galois representations, J. Amer. Math. Soc. 12 (1999), 521-567.

[82] D. Cox, Primes of the form $x^{2}+n y^{2}$, Wiley and Sons, New York, 1989.

[83] P. Deligne, Formes modulaires et reṕresentations $\ell$-adiques, Sém. Bourbaki 355 (1969).

[84] F. Diamond and J. Shurman, A first course in modular forms, Springer-Verlag, New York, 2005.

[85] L. Dragonette, Some asymptotic formulae for the mock theta series of Ramanujan, Trans. Amer. Math. Soc. 72 No. 3 (1952), pages 474-500.

[86] W. Duke, Hyperbolic distribution problems and half-integral weight Maass forms, Invent. Math. 92 (1988), no. 1, 73-90.

[87] W. Duke, Modular functions and the uniform distribution of CM points, Math. Ann. 334 (2006), no. 2, pages 241-252.

[88] W. Duke, O. Imamoḡlu, and A. Toth, Cycle integrals of the $j$-function and weakly harmonic modular forms, preprint.

[89] W. Duke and P. Jenkins, Integral traces of singular values of weak Maass forms, Algebra and Number Theory, 2 (2008), pages 573-593.

[90] F. Dyson, Some guesses in the theory of partitions, Eureka (Cambridge) 8 (1944), pages 10-15.

[91] F. Dyson, A walk through Ramanujan's garden, Ramanujan revisited (UrbanaChampaign, Ill. 1987), Academic Press, Boston, 1988, pages 7-28.

[92] T. Eguchi and K. Hikami, Superconformal algebras and mock theta functions, preprint.

[93] M. Eichler and D. Zagier, The theory of Jacobi forms, Birkhaüser, Boston, 1985.

[94] A. Folsom, A short proof of the Mock Theta Conjectures using Maass forms, Proc. Amer. Math. Soc. 136 (2008), pages 4143-4149.

[95] A. Folsom and R. Masri, Limiting distribution of traces of Maass-Poincaré series, preprint.

[96] A. Folsom and K. Ono, Duality involving the mock theta function $f(q)$, J. London Math. Soc. 77 (2008), pages 320-334.

[97] S. Garthwaite, On questions of congruence and size for modular forms and MaassPoincaré series, Ph.D. Thesis (Advisor: K. Ono), University of Wisconsin, 2007.

[98] S. Garthwaite, Vector-valued Maass-Poincaré series, Proc. Amer. Math. Soc., 136 (2008) no. 2, pages 427-436.

[99] F. Garvan, D. Kim, and D. Stanton, Cranks and t-cores, Invent. Math. 101 (1990), pages $1-17$.

[100] D. Goldfeld and P. Sarnak, Sums of Kloosterman sums, Invent. Math. 71 (1983), pages 243-250. 
[101] H. Göllnitz, Partitionen mit differenzenbedingungen, J. reine angew. Math. 225 (1967), pages 154-190.

[102] B. Gordon, Some continued fractions of the Rogers-Ramanujan type, Duke Math. J. 32 (1965), pages 741-748.

[103] B. Gordon and R. McIntosh, Some eighth order mock theta functions, J. London Math. Soc. 62 No. 2 (2000), pages 321-335.

[104] B. Gordon and R. McIntosh, Modular transformations of Ramanujan's fifth and seventh order mock theta functions, Ramanujan J. 7 (2003), pages 193-222.

[105] L. Göttsche and D. Zagier, Jacobi forms and the structure of Donaldson invariants for 4-manifolds with $b_{+}=1$, Selecta Math. (N.S.) 4 (1998), pages 69-115.

[106] B. Gross and K. Keating, On the intersection of modular correspondences, Invent. Math. 112 (1993), 225-245.

[107] B. Gross, W. Kohnen, and D. Zagier, Heegner points and derivatives of L-series. II, Math. Ann. 278 (1987), pages 497-562.

[108] B. Gross and D. Zagier, Heegner points and derivatives of L-series, Invent. Math. 84 (1986), pages 225-320.

[109] P. Guerzhoy, On weak Maass-Modular grids of even integral weights, Math. Res. Lettrs., accepted for publication.

[110] G. H. Hardy, Srinivasa Ramanujan (188\%-1920), Collected papers of Ramanujan (Ed. G. H. Hardy, et. al.), Cambridge Univ. Press, Cambrdige, 1927, pages xxixxxvi.

[111] G. H. Hardy, Ramanujan: Twelve lectures on subjects suggested by his life and work, Cambridge Univ. Press, Cambridge, 1940.

[112] G. H. Hardy (with a foreward by C. P. Snow), A mathematician's apology, Cambridge Univ. Press, Cambridge, 1992.

[113] G. H. Hardy and S. Ramanujan, Une formule asymptotique pour le nombre des partitions de $n$ [Comptes Rendus, 2 Jan. 1917] (French) [An asymptotic formula for the number of partitions of $n$ ] Collected papers of Srinivasa Ramanujan, pages 239-241, AMS Chelsea Publ., Providence, RI, 2000.

[114] G. H. Hardy and S. Ramanujan, Asymptotic formulae in combinatory analysis [Proc. London Math. Soc. (2) 16 (1917), Records for 1 March 1917]. Collected papers of Srinivasa Ramanujan, 244, AMS Chelsea Publ., Providence, RI, 2000.

[115] E. Hecke, Mathematische Werke, Vandenhoeck \& Ruprecht, Third edition, Göttingen, 1983.

[116] D. A. Hejhal, The Selberg trace formula for $P S L(2, \mathbb{R})$, Springer Lect. Notes in Math. 548, Springer-Verlag, Berlin 1976.

[117] D. A. Hejhal, The Selberg trace formula for $P S L(2, \mathbb{R})$, Springer Lect. Notes in Math. 1001, Springer-Verlag, Berlin, 1983.

[118] D. Hickerson, A proof of the mock theta conjectures, Invent. Math. 94 (1988), pages 639-660.

[119] D. Hickerson, On the seventh order mock theta functions, Invent. Math. 94 No. 3 (1988), pages 661-677.

[120] K. Hikami, Mock (false) theta functions as quantum invariants, Regul. Chaotic Dyn. 10 (2005), pages 163-174.

[121] K. Hikami, Transformation formula of the "second" order mock theta function, Lett. Math. Phys. 75 (2006), pages 93-98.

[122] K. Hikami, Hecke type formula for the unified Witten-Reshetikhin invariants as higher order mock theta functions, Int. Math. Res. Not. 7 (2007), 32 pp.

[123] F. Hirzebruch and D. Zagier, Intersection numbers of curves on Hilbert modular surfaces and modular forms with Nebentypus, Invent. Math. 36 (1976), pages 57113.

[124] H. Iwaniec, Non-holomorphic modular forms and their applications, Modular forms (ed. R. Rankin), Ellis Horwood Ltd., Chichester, 1984. 
[125] H. Iwaniec, Topics in classical automorphic forms, Grad. Studies in Math. 17, Amer. Math. Soc., Providence, RI, 1997.

[126] H. Iwaniec, Spectral methods of automorphic forms, Graduate Studies in Math., 53, Amer. Math. Soc., Providence, RI, 2002.

[127] P. Jenkins, Traces of singular moduli, modular forms, and Maass forms, Ph.D. Thesis (Advisor: K. Ono), University of Wisconsin, 2006.

[128] P. Jenkins, Kloosterman sums and traces of singular moduli, J. Number Th. 117 (2006), pages 301-314.

[129] V. G. Kac, Infinite dimensional Lie algebras, Cambridge Univ. Press, Cambridge, 1990.

[130] V. G. Kac and M. Wakimoto, Integrable highest weight modules over affine superalgebras and number theory, Lie theory and geometry, Progr. Math. 123 Birkhäuser, Boston, 1994, pages 415-456.

[131] V. G. Kac and M. Wakimoto, Integrable highest weight modules over affine superalgebras and Appell's function, Comm. Math. Phys. 215 (2001), pages 631-682.

[132] S.-Y. Kang, Mock Jacobi forms in basic hypergeometric series, Compositio Math. 145 (2009), pages 553-565.

[133] R. Kanigel, The man who knew infinity: A Life of the Genius Ramanujan, Washington Square, 1992.

[134] N. Koblitz, Introduction to elliptic curves and modular forms, Springer-Verlag, New York, 1993.

[135] W. Kohnen, Newforms of half-integral weight, J. Reine Angew. Math. 333 (1982), $32-72$.

[136] W. Kohnen, Fourier coefficients of modular forms of half-integral weight, Math. Ann. 271 (1985), 237-268.

[137] W. Kohnen and D. Zagier, Values of L-series of modular forms at the center of the critical strip, Invent. Math. 64 (1981), no. 2, pages 175-198.

$[138]$ V. A. Kolyvagin, Finiteness of $E(\mathbb{Q})$ and the Tate-Shafarevich group of $E(\mathbb{Q})$ for a subclass of Weil curves (Russian), Izv. Akad. Nauk, USSR, ser. Matem. 52 (1988), 522-540.

[139] D. Kubert and S. Lang, Modular units, Grundlehren der Mathematischen Wissenschaften [Fundamental Principles of Mathematical Science], 244, SpringerVerlag, New York-Berlin, 1981.

[140] S. Kudla, Central derivatives of Eisenstein series and height pairings, Ann. of Math. (2) 146 (1997), 545-646.

[141] S. Kudla, Special cycles and derivatives of Eisenstein series, in Heegner points and Rankin L-series, Math. Sci. Res. Inst. Publ. 49, Cambridge University Press, Cambridge, 2004.

[142] S. Kudla, M. Rapoport, and T. H. Yang, Modular forms and special cycles on Shimura curves, Annals of Math. Studies series, vol 161, Princeton Univ. Publ., 2006.

[143] S. Lang, Introduction to modular forms, Springer-Verlag, Berlin, 1995.

[144] R. Lawrence and D. Zagier, Modular forms and quantum invariants of 3-manifolds, Asian J. Math. 3 (1999), pages 93-107.

[145] J. Lepowsky and R. L. Wilson, A new family of algebras underlying the RogersRamanujan identities and generalizations, Proc. Natl. Acad. Sci., USA 78 (1981), pages $7254-7258$.

[146] M. Lerch, Bemerkungen zur Theorie der elliptischen Functionen, Jahrbuch über die Fortschritte der Mathematik 24 (1892), pages 442-445.

[147] M. Lerch, Poznámky $k$ theorii funkcí elliptických, Rozpravy České Akademie Císaře Františka Josefa pro vědy, slovesnost a umění v praze (IIC1) I 24 (1892), pages 465-480. 
[148] J. Lewis and D. Zagier, Period functions for Maass wave forms. I., Ann. Math. 153 (2001), pages 191-258.

[149] H. Maass, Über eine neue Art von nichtanalytischen automorphen Funktionen und die Bestimmung Dirichletscher Reihen durch Funktionalgleichungen, Math. Ann. 121 (1949), pages 141-183.

[150] H. Maass, Die Differentialgleichungen in der Theorie der elliptische Modulfunktionen, Math. Ann. 125 (1953), pages 235-263.

[151] K. Mahlburg, Partition congruences and the Andrews-Garvan-Dyson crank, Proc. Natl. Acad. Sci. USA 102 (2005), no. 43, pages 15373-15376.

[152] A. Malmendier and K. Ono, SO(3)-Donaldson invariants of $\mathbb{C} P^{2}$ and mock theta functions, submitted for publication.

[153] A. Mellit and S. Okada, Joyce invariants for K3 surfaces and mock theta functions, preprint.

[154] A. Miller and A. Pixton, Arithmetic traces of non-holomorphic modular invariants, Int. J. of Number Theory, accepted for publication.

[155] T. Miyake, Modular forms, Springer-Verlag, Berlin, 2006.

[156] M. Monks, Number theoretic properties of generating functions related to Dyson's rank for partitions into distinct parts, preprint.

[157] L. J. Mordell, The value of the definite integral $\int_{-\infty}^{\infty} \frac{e^{a t^{2}+b t}}{e^{c t}+d} d t$, Quarterly J. of Math. 68 (1920), pages 329-342.

[158] L. J. Mordell, The definite integral $\int_{-\infty}^{\infty} \frac{e^{a t^{2}+b t}}{e^{c t}+d} d t$ and the analytic theory of numbers, Acta Math. 61 (1933), pages 323-360.

[159] W. Nahm, Conformal field theory and torsion elements of the Bloch group, Frontiers of number theory, physics, and geometry I, Springer-Verlag, Berlin, 2007, pages $67-132$.

[160] D. Niebur, A class of nonanalytic automorphic functions, Nagoya Math. J. 52 (1973), pages 133-145.

[161] D. Niebur, Construction of automorphic forms and integrals, Trans. Amer. Math. Soc. 191 (1974), pages 373-385.

[162] K. Ono, Distribution of the partition function modulo $m$, Ann. of Math. (2) 151 (2000), no. 1, pages 293-307.

[163] K. Ono, Arithmetic of the partition function, Special functions 2000: current perspective and future directions (Tempe, AZ), NATO Sci. Ser. II Math. Phys. Chem., 30, Kluwer Acad. Publ., Dordrecht, 2001, pages 243-253.

[164] K. Ono, The web of modularity: arithmetic of the coefficients of modular forms and q-series, CBMS Regional Conference Series in Mathematics, 102, Amer. Math. Soc., Providence, RI, 2004.

[165] K. Ono, Honoring a gift from Kumbakonam, Notices Amer. Math. Soc. 53 (2006), no. 6, pages $640-651$.

[166] K. Ono, A mock theta function for the Delta-function, Proceedings of the 2007 Integers Conference, de Gruyter, Berlin, 2009, pages 141-156.

[167] H. Petersson, Über die Entwicklungskoeffizienten der automorphen Formen, Acta Math. 58 (1932), pages 169-215.

[168] H. Rademacher, On the expansion of the partition function in a series, Ann. of Math. (2) 44, (1943), pages 416-422.

[169] H. Rademacher, Topics in analytic number theory, Die Grundlehren der mathematischen Wissenschaften, Band 169, Springer Verlag New York-Heidelberg, 1973.

[170] H. Rademacher and H. S. Zuckerman, On the Fourier coefficients of certain modular forms of positive dimension, Ann. of Math. (2) 39 (1938), no. 2, pages 433-462.

[171] S. Ramanujan, Problem 584, J. Indian Math. Soc. 6 (1914), pages 199-200.

[172] S. Ramanujan, On certain arithmetical functions, Trans. Camb. Phil. Soc. 22 (1916), pages 159-184. 
[173] S. Ramanujan, Some properties of $p(n)$, the number of partitions of $n$, [Proc. Cambridge Philos. Soc. 19 (1919), pages 207-210]. Collected papers of Srinivasa Ramanujan, 210-213, AMS Chelsea Publ., Providence, RI, 2000.

[174] S. Ramanujan, Congruence properties of partitions, Math. Z. 9 (1921), no. 1-2, pages $147-153$.

[175] S. Ramanujan, Collected papers of Ramanujan, Cambridge Univ. Press, Cambridge, 1927.

[176] S. Ramanujan, The lost notebook and other unpublished papers, Narosa, New Delhi, 1988.

[177] R. A. Rankin, Modular forms and functions, Cambridge Univ. Press, CambridgeNew York-Melbourne, 1977.

[178] R. Rhoades, Linear relations among Poincaré series, preprint.

[179] R. Rhoades, Interplay between Weak Maass Forms and Modular Forms and Statistical Properties of Number Theoretic Objects, Ph.D. thesis (Advisor: K. Ono), University of Wisconsin, 2008.

[180] K. Ribet, Galois representations attached to eigenforms with Nebentypus, Springer Lect. Notes in Math. 601, (1977), pages 17-51.

[181] W. Roelcke, Automorphe formen in der hyperbolishce Ebene, I, Math. Ann. 167 (1966), pages 292-337.

[182] L. J. Rogers, Second memoir on the expansion of certain infinite products, Proc. London Math. Soc. 25 (1894), pages 318-343.

[183] L. J. Rogers, On two theorems of combinatory analysis and some allied identities, Proc. London Math. Soc. 16 (1917), pages 315-336.

[184] L. J. Rogers, Proof of certain identities in combinatory analysis, Proc. Cambridge Philos. Soc. 19 (1919), pages 211-214.

[185] P. Sarnak, Some applications of modular forms, Cambridge Tracts in Math, 99, Cambridge Univ. Press, Cambridge, 1990.

[186] B. Schoeneberg, Elliptic modular functions: an introduction, Springer-Verlag, New York-Heidelberg, 1974.

[187] J.-P. Serre, Congruences et formes modulaires (d'apres H. P. F. Swinnerton-Dyer), Sem. Bourbaki 416 (1971-1972), pages 74-88.

[188] J.-P. Serre, Divisibilité de certaines fonctions arithmétiques, Enseign. Math. 22 (1976), pages 227-260.

[189] J.-P. Serre and H. Stark, Modular forms of weight 1/2, Springer Lect. Notes 627, Springer-Verlag, Berlin, (1977), pages 27-67.

[190] A. Selberg, Über die Mock-Thetafunktionen siebenter Ordnung, Arch. Math. Natur. idenskab, 41 (1938), pages 3-15 (see also Coll. Papers, I, pages 22-37).

[191] A. Selberg, Harmonic analysis and discontinuous groups in weakly symmetric Riemannian spaces, J. Indian Math. Soc. 20 (1956), pages 47-87.

[192] G. Shimura, On modular forms of half integral weight, Ann. of Math. 97 (1973), pages $440-481$.

[193] G. Shimura, Introduction to the arithmetic theory of automorphic functions, Reprint of the 1971 original. Publications of the Math. Soc. of Japan, 11. Kan Memorial Lectures, 1. Princeton Univ. Press, Princeton, NJ, 1994.

[194] J. Silverman, The arithmetic of elliptic curves, Springer-Verlag, New York, 1986.

[195] L. J. Slater, Further identities of the Rogers-Ramanujan type, Proc. London Math. Soc. Ser. no. 254 (1952), pages 147-167.

[196] W. Stein, Modular forms, a computational approach, Amer. Math. Soc., Providence, RI, 2007.

[197] H. P. F. Swinnerton-Dyer, On $\ell$-adic representations and congruences for coefficients of modular forms, Springer Lect. Notes. 350 (1973), pages 1-55.

[198] R. Taylor and A. Wiles, Ring-theoretic properties of certain Hecke algebras, Ann. of Math. 141 (1995), 553-572. 
[199] A. Terras, Harmonic analysis on symmetric spaces and applications, I, SpringerVerlag, New York, 1985.

[200] A. Terras, Harmonic analysis and symmetric spaces and applications, II, SpringerVerlag, Berlin, 1988.

[201] S. Treneer, Congruences for the coefficients of weakly holomorphic modular forms, Proc. London Math. Soc., 93 (2006), pages 304-324.

[202] J. Tunnell, A classical Diophantine problem and modular forms of weight $3 / 2$ Invent. Math. 72 (1983), no. 2, pages 323-334.

[203] A. Venkov, Spectral theory of automorphic functions, Kluwer Acad. Publ., Dordrecht, 1990.

[204] M. Wakimoto, Representation theory of affine superalgebras at the critical level, Proc. Int. Congress of Math., Vol. II, (Berlin, 1998), Doc. Math. 1998, Extra Vol., II, pages 605-614.

[205] J.-L. Waldspurger, Sur les coefficients de Fourier des formes modulaires de poids demi-entier, J. Math. Pures Appl. (9) 60 (1981), no. 4, pages 375-484.

[206] G. N. Watson, The final problem: An account of the mock theta functions, J. London Math. Soc. 2 (2) (1936), pages 55-80.

[207] G. N. Watson, The mock theta functions (2), Proc. London Math. Soc. (2) 42 (1937), pages 274-304.

[208] G. N. Watson, Ramanujan's Vermutung über Zerfällungsanzahlen, J. Reine Angew. Math. 179 (1938), pages 97-128.

[209] A. Wiles, Modular elliptic curves and Fermat's last theorem, Ann. of Math. 141 (1995), 443-551.

[210] D. Zagier, Nombres de classes et formes modulaires de poids 3/2, C. R. Acad. Sci. Paris Sér. A-B 281 (1975), pages 883-886.

[211] D. Zagier, Vassiliev invariants and a strange identity related to the Dedekind etafunction, Topology 40 (2001), pages 945-960.

[212] D. Zagier, Traces of singular moduli, Motives, Polylogarithms and Hodge Theory, Part I. International Press Lecture Series (Eds. F. Bogomolov and L. Katzarkov), International Press, 2002, pages 211-244.

[213] D. Zagier, Ramanujan's mock theta functions and their applications [d'après Zwegers and Bringmann-Ono], Séminaire Bourbaki, 60éme année, 2006-2007, no. 986.

[214] S. Zhang, Heights of Heegner cycles and derivatives of L-series, Invent. Math. 130 (1997), pages 99-152.

[215] S. Zhang, Heights of Heegner points on Shimura curves, Ann. of Math. 153 (2001), pages $27-147$.

[216] H. S. Zuckerman, On the coefficients of certain modular forms belonging to subgroups of the modular group, Trans. Amer. Math. Soc., 45 (1939), pages 298-321.

[217] H. S. Zuckerman, On the expansions of certain modular forms of positive dimension, Amer. J. Math. 62 (1940), pages 127-152.

[218] S. P. Zwegers, Mock $\vartheta$-functions and real analytic modular forms, $q$-series with applications to combinatorics, number theory, and physics (Ed. B. C. Berndt and K. Ono), Contemp. Math. 291, Amer. Math. Soc., (2001), pages 269-277.

[219] S. P. Zwegers, Mock theta functions, Ph.D. Thesis (Advisor: D. Zagier), Universiteit Utrecht, 2002.

Department of Mathematics, University of Wisconsin, Madison, Wisconsin 53706 USA

E-mail address: ono@math.wisc.edu 